\title{
Planck early results. VIII. The all-sky early Sunyaev-Zeldovich cluster sample ${ }^{\star, \star \star}$
}

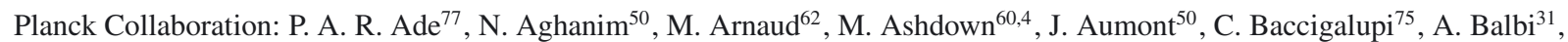
A. J. Banday ${ }^{85,7,68}$, R. B. Barreiro ${ }^{57}$, M. Bartelmann ${ }^{83,68}$, J. G. Bartlett ${ }^{3,58}$, E. Battaner ${ }^{88}$, R. Battye ${ }^{59}$, K. Benabed ${ }^{51}$, A. Benoît ${ }^{49}$, J.-P. Bernard ${ }^{85,7}$, M. Bersanellii ${ }^{28,44}$, R. Bhatia ${ }^{5}$, J. J. Bock ${ }^{58,8}$, A. Bonaldi ${ }^{40}$, J. R. Bond ${ }^{6}$, J. Borrill ${ }^{66,79}$, F. R. Bouchet ${ }^{51}$, M. L. Brown ${ }^{4,60}$, M. Bucher ${ }^{3}$,

C. Burigana ${ }^{43}$, P. Cabella ${ }^{31}$, C. M. Cantalupo ${ }^{66}$, J.-F. Cardoso ${ }^{63,3,51}$, P. Carvalho ${ }^{4}$, A. Catalano ${ }^{3,61}$, L. Cayón ${ }^{21}$, A. Challinor ${ }^{54,60,11}$,

A. Chamballu ${ }^{47}$, R.-R. Chary ${ }^{48}$, L.-Y Chiang ${ }^{53}$, C. Chiang ${ }^{20}$, G. Chon ${ }^{69,4}$, P. R. Christensen ${ }^{72,32}$, E. Churazov ${ }^{68,78}$, D. L. Clements ${ }^{47}$, S. Colafrancesco ${ }^{41}$, S. Colombi ${ }^{51}$, F. Couchot ${ }^{65}$, A. Coulais ${ }^{61}$, B. P. Crill ${ }^{58,73}$, F. Cuttaia ${ }^{43}$, A. Da Silva ${ }^{10}$, H. Dahle ${ }^{55,9}$, L. Danese ${ }^{75}$, R. J. Davis ${ }^{59}$, P. de Bernardis ${ }^{27}$, G. de Gasperis ${ }^{31}$, A. de Rosa ${ }^{43}$, G. de Zotti ${ }^{40,75}$, J. Delabrouille ${ }^{3}$, J.-M. Delouis ${ }^{51}$, F.-X. Désert ${ }^{46}$, C. Dickinson ${ }^{59}$, J. M. Diego ${ }^{57}$, K. Dolag ${ }^{68}$, H. Dole ${ }^{50}$, S. Donzelli ${ }^{44,55}$, O. Doré ${ }^{58,8}$, U. Dörl $^{68}$, M. Douspis ${ }^{50}$, X. Dupac ${ }^{36}$, G. Efstathiou ${ }^{54}$, P. Eisenhardt ${ }^{58}$, T. A. Enßlin ${ }^{68}$,

F. Feroz ${ }^{4}$, F. Finelli ${ }^{43}$, I. Flores-Cacho ${ }^{56,33}$, O. Forni ${ }^{85,7}$, P. Fosalba ${ }^{52}$, M. Frailis ${ }^{42}$, E. Franceschi ${ }^{43}$, S. Fromenteau ${ }^{3,50}$, S. Galeotta ${ }^{42}$, K. Ganga $^{3,48}$, R. T. Génova-Santos ${ }^{56,33}$, M. Giard ${ }^{85,7}$, G. Giardino ${ }^{37}$, Y. Giraud-Héraud ${ }^{3}$, J. González-Nuevo ${ }^{75}$, R. González-Riestra ${ }^{35}$, K. M. Górski ${ }^{58,90}$,

K. J. B. Grainge ${ }^{4,60}$, S. Gratton ${ }^{60,54}$, A. Gregorio ${ }^{29}$, A. Gruppuso ${ }^{43}$, D. Harrison ${ }^{54,60}$, P. Heinämäki ${ }^{82}$, S. Henrot-Versillée ${ }^{65}$,

C. Hernández-Monteagudo ${ }^{68}$, D. Herranz ${ }^{57}$, S. R. Hildebrandt ${ }^{8,64,56}$, E. Hivon ${ }^{51}$, M. Hobson ${ }^{4}$, W. A. Holmes ${ }^{58}$, W. Hovest ${ }^{68}$, R. J. Hoyland ${ }^{56}$, K. M. Huffenberger ${ }^{89}$, G. Hurier ${ }^{64}$, N. Hurley-Walker ${ }^{4}$, A. H. Jaffe ${ }^{47}$, W. C. Jones ${ }^{20}$, M. Juvela ${ }^{19}$, E. Keihänen ${ }^{19}$, R. Keskitalo ${ }^{58,19}$, T. S. Kisner ${ }^{66}$, R. Kneiss ${ }^{34,5}$, L. Knox ${ }^{23}$, H. Kurki-Suonio ${ }^{19,39}$, G. Lagache ${ }^{50}$, J.-M. Lamarre ${ }^{61}$, A. Lasenby ${ }^{4,60}$, R. J. Laureijs ${ }^{37}$, C. R. Lawrence ${ }^{58}$, M. Le Jeune ${ }^{3}$, S. Leach $^{75}$, R. Leonardi ${ }^{36,37,24}$, C. Li ${ }^{67,68}$, A. Liddle ${ }^{18}$, P. B. Lilje ${ }^{55,9}$, M. Linden-Vørnle ${ }^{13}$, M. López-Caniego ${ }^{57}$, P. M. Lubin ${ }^{24}$,

J. F. Macías-Pérez ${ }^{64}$, C. J. MacTavish ${ }^{60}$, B. Maffei ${ }^{59}$, D. Maino ${ }^{28,44}$, N. Mandolesi ${ }^{43}$, R. Mann ${ }^{76}$, M. Maris ${ }^{42}$, F. Marleau ${ }^{15}$,

E. Martínez-González ${ }^{57}$, S. Masi ${ }^{27}$, S. Matarrese ${ }^{26}$, F. Matthai ${ }^{68}$, P. Mazzotta ${ }^{31}$, S. Mei ${ }^{84,38,8}$, P. R. Meinhold ${ }^{24}$, A. Melchiorri ${ }^{27}$, J.-B. Melin ${ }^{12}$, L. Mendes ${ }^{36}$, A. Mennella ${ }^{28,42}$, S. Mitra ${ }^{58}$, M.-A. Miville-Deschênes ${ }^{50,6}$, A. Moneti ${ }^{51}$, L. Montier ${ }^{85,7}$, G. Morgante ${ }^{43}$, D. Mortlock ${ }^{47}$,

D. Munshi ${ }^{77,54}$, A. Murphy ${ }^{71}$, P. Naselsky ${ }^{72,32}$, F. Nati ${ }^{27}$, P. Natoli ${ }^{30,2,43}$, C. B. Netterfield ${ }^{15}$, H. U. Nørgaard-Nielsen ${ }^{13}$, F. Noviello ${ }^{50}$, D. Novikov ${ }^{47}$,

I. Novikov ${ }^{72}$, M. Olamaie ${ }^{4}$, S. Osborne ${ }^{80}$, F. Pajot ${ }^{50}$, F. Pasian ${ }^{42}$, G. Patanchon ${ }^{3}$, T. J. Pearson ${ }^{8,48}$, O. Perdereau ${ }^{65}$, L. Perotto ${ }^{64}$, F. Perrotta ${ }^{75}$,

F. Piacentini ${ }^{27}$, M. Piat ${ }^{3}$, E. Pierpaoli ${ }^{17}$, R. Piffaretti ${ }^{62,12}$, S. Plaszczynski ${ }^{65}$, E. Pointecouteau ${ }^{85,7}$, G. Polenta ${ }^{2,41}$, N. Ponthieu ${ }^{50}$, T. Poutanen ${ }^{39,19,1}$, G. W. Pratt ${ }^{62}$, G. Prézeau ${ }^{8,58}$, S. Prunet ${ }^{51}$, J.-L. Puget ${ }^{50}$, J. P. Rachen ${ }^{68}$, W. T. Reach ${ }^{86}$, R. Rebolo $^{56,33}$, M. Reinecke ${ }^{68}$, C. Renault ${ }^{64}$, S. Ricciardi ${ }^{43}$,

T. Riller ${ }^{68}$, I. Ristorcelli ${ }^{85,7}$, G. Rocha ${ }^{58,8}$, C. Rosset $^{3}$, J. A. Rubiño-Martín ${ }^{56,33}$, B. Rusholme ${ }^{48}$, E. Saar ${ }^{81}$, M. Sandri ${ }^{43}$, D. Santos ${ }^{64}$,

R. D. E. Saunders ${ }^{4,60}$, G. Savini ${ }^{74}$, B. M. Schaefer ${ }^{83}$, D. Scott ${ }^{16}$, M. D. Seiffert ${ }^{58,8}$, P. Shellard ${ }^{11}$, G. F. Smoot ${ }^{22,66,3}$, A. Stanford ${ }^{23}$, J.-L. Starck ${ }^{62,12}$, F. Stivoli ${ }^{45}$, V. Stolyarov ${ }^{4}$, R. Stompor ${ }^{3}$, R. Sudiwala ${ }^{77}$, R. Sunyaev ${ }^{68,78}$, D. Sutton ${ }^{54,60}$, J.-F. Sygnet ${ }^{51}$, N. Taburet ${ }^{50}$, J. A. Tauber ${ }^{37}$, L. Terenzi ${ }^{43}$, L. Toffolatti ${ }^{14}$, M. Tomasi ${ }^{28,44}$, J.-P. Torre ${ }^{50}$, M. Tristram ${ }^{65}$, J. Tuovinen ${ }^{70}$, L. Valenziano ${ }^{43}$, L. Vibert ${ }^{50}$, P. Vielva $^{57}$, F. Villa $^{43}$, N. Vittorio $^{31}$, L. A. Wade ${ }^{58}$, B. D. Wandelt ${ }^{51,25}$, J. Weller ${ }^{87}$, S. D. M. White ${ }^{68}$, M. White ${ }^{22}$, D. Yvon ${ }^{12}$, A. Zacchei ${ }^{42}$, and A. Zonca ${ }^{24}$

(Affiliations can be found after the references)

Received 7 January 2011 / Accepted 15 May 2011

\section{ABSTRACT}

We present the first all-sky sample of galaxy clusters detected blindly by the Planck satellite through the Sunyaev-Zeldovich (SZ) effect from its six highest frequencies. This early SZ (ESZ) sample is comprised of 189 candidates, which have a high signal-to-noise ratio ranging from 6 to 29. Its high reliability (purity above 95\%) is further ensured by an extensive validation process based on Planck internal quality assessments and by external cross-identification and follow-up observations. Planck provides the first measured SZ signal for about $80 \%$ of the 169 previouslyknown ESZ clusters. Planck furthermore releases 30 new cluster candidates, amongst which 20 meet the ESZ signal-to-noise selection criterion. At the submission date, twelve of the 20 ESZ candidates were confirmed as new clusters, with eleven confirmed using XMM-Newton snapshot observations, most of them with disturbed morphologies and low luminosities. The ESZ clusters are mostly at moderate redshifts $(86 \%$ with $z$ below 0.3 ) and span more than a decade in mass, up to the rarest and most massive clusters with masses above $1 \times 10^{15} M_{\odot}$.

Key words. cosmology: observations - galaxies: clusters: general - catalogs

\section{Introduction}

Galaxy clusters provide valuable information on cosmology, from the nature of dark energy to the physics that drives galaxy and structure formation. The main baryonic component in these

\footnotetext{
^ Corresponding author: M. Douspis, e-mail: marian.douspis@ias.u-psud.fr $\star \star$ Appendix is available in electronic form at http://www . aanda.org
}

dark matter dominated objects is a hot, ionised intra-cluster medium (ICM). The ICM can be studied both in the X-ray and through the Sunyaev-Zeldovich effect (SZ) (Sunyaev \& Zeldovich 1972; Sunyaev \& Zeldovich 1980), a fairly new and highly promising technique that has made tremendous progress in recent years since its first observations (Birkinshaw \& Gull 1978); see also Rephaeli (1995); Birkinshaw (1999); Carlstrom et al. (2002). 
The SZ effect is undoubtedly the best known and most studied secondary contribution, due to cosmic structure, that is imprinted on the cosmic microwave background (CMB) after decoupling (for a review of secondary anisotropies see Aghanim et al. 2008). It is caused by the inverse Compton interaction between the CMB photons and the free electrons of the hot ICM. It can be broadly subdivided into the thermal SZ (TSZ) effect, where the photons are scattered by the random motion of thermal electrons, and the kinetic SZ (KSZ) effect caused by the bulk motion of the electrons. In the former case, the scattered CMB photons have a unique spectral dependence, whereas the final spectrum remains Planckian in the case of the KSZ effect.

The SZ effect offers a number of advantages for cluster studies. First, the Compton $y$ parameter, which measures the integral of the gas pressure along the line of sight and sets the amplitude of the SZ signal, does not suffer from cosmological surfacebrightness dimming. This implies that the SZ effect is an efficient method for finding high-redshift clusters. Second, the total SZ signal $Y$, integrated over the cluster's angular extent, directly measures the total thermal energy of the gas and as such is expected to correlate closely (i.e., with a tight scatter in the scaling relation) with total cluster mass. This fact is borne out both by numerical simulations (Borgani 2006; da Silva et al. 2001; Motl et al. 2005; Pfrommer et al. 2007) and indirectly from X-ray observations (Nagai et al. 2007; Arnaud et al. 2007; Vikhlinin et al. 2009) using $Y_{X}$, the product of the gas mass and mean temperature giving an X-ray analogue of the integrated SZ Compton parameter first introduced by Kravtsov et al. (2006). This contrasts with the X-ray luminosity which, at a given mass, is very sensitive to the cluster's thermodynamical state, for instance due to a recent merger event or in the presence of a strong cooling core. Hence SZ surveys are expected to provide clean cluster samples over a wide range of redshifts, in the sense of being close to an unbiased mass-limited selection. These are key properties for statistical studies with clusters, either to constrain cosmological models (e.g., from the evolution of the mass function) or to probe the physics of structure formation (e.g., from cluster scaling and structural properties).

For these reasons, alongside the efforts developed to measure CMB anisotropies many pioneering instruments were used or developed to observe the SZ effect and use it as new observational probe of cluster physics, large-scale structure, and the cosmological model. The first observations of the SZ effect, targeted at specific X-ray selected clusters, were performed using interferometric or single-dish experiments mostly observing in the Rayleigh-Jeans part of the spectrum: the Ryle Telescope at $15 \mathrm{GHz}$ (Jones et al. 1993), the OVRO $5 \mathrm{~m}$ telescope at $32 \mathrm{GHz}$ (Birkinshaw \& Hughes 1994), the SuZIE array at $140 \mathrm{GHz}$ (Holzapfel et al. 1997), BOLOCAM at 143 and $265 \mathrm{GHz}$ (Glenn et al. 1998), the Diabolo array on IRAM $30 \mathrm{~m}$ telescope at $140 \mathrm{GHz}$ (Pointecouteau et al. 1999), MITO at 143, 214, 272, and $353 \mathrm{GHz}$ (De Petris et al. 1999), the Nobeyama $45 \mathrm{~m}$ telescope at $21 \mathrm{GHZ}, 43 \mathrm{GHz}$ and $150 \mathrm{GHz}$ (Komatsu et al. 1999), the BIMA array at $30 \mathrm{GHz}$ (Dawson et al. 2001), ACBAR at 150 and $220 \mathrm{GHz}$ (Gómez et al. 2003), CBI working between 25 and $36 \mathrm{GHz}$ (Udomprasert et al. 2004), VSA at $30 \mathrm{GHz}$ (Lancaster et al. 2005), the Atacama Pathfinder Experiment (APEX) SZ Camera at $150 \mathrm{GHz}$ (Dobbs et al. 2006), the SZ Array at $30 \mathrm{GHz}$ (Muchovej et al. 2007), AMI at $15 \mathrm{GHz}$ (Zwart et al. 2008), and AMIBA at $90 \mathrm{GHz}$ (Wu et al. 2008); see Birkinshaw \& Lancaster (2005) for a review of observational techniques. Measurements of the SZ effect were further made or attempted in the Wien part of the spectrum with PRONAOS (Lamarre et al. 1998), SCUBA
(Zemcov et al. 2007), and more recently with the Herschel Space Observatory (Zemcov et al. 2010).

These experiments have not only allowed us to accumulate SZ measurements for about a hundred clusters, but have also laid the groundwork for SZ-based studies of clusters and of cosmology. In combination with other observations, especially in X-rays, they were used to measure cosmological parameters such as the Hubble constant, and to probe the distance-duality relation between the angular-diameter and luminosity distances, bulk flows, and the cluster gas mass fraction (e.g., Silk \& White 1978; Kobayashi et al. 1996; Grego et al. 2001; Reese et al. 2002; Uzan et al. 2004; Ameglio et al. 2006; Bonamente et al. 2006; Kashlinsky et al. 2008). The SZ effect has also been used to characterise the clusters themselves, as it can potentially measure their radial peculiar velocities (Benson et al. 2003). The relativistic corrections to the SZ effect (e.g., Itoh et al. 1998) can be used to measure the gas temperature directly for massive clusters (Pointecouteau et al. 1998). The spectral signature of the SZ effect can in principle even probe the electron gas distribution and constrain any non-thermal electron population in the ICM (Colafrancesco et al. 2003; Shimon \& Rephaeli 2004). The SZ effect can also be used as a tracer of the WHIM diffuse gas (Génova-Santos et al. 2005; Battistelli et al. 2006). Moreover multi-frequency SZ measurements might provide a novel way of constraining the $\mathrm{CMB}$ temperature and its evolution with redshift (Battistelli et al. 2002; Horellou et al. 2005; Luzzi et al. 2009).

Deep surveys covering hundreds of square degrees and capable of detecting many tens to hundreds of clusters, performed by the South Pole Telescope (SPT) (Carlstrom et al. 2011) and the Atacama Cosmology Telescope (ACT) (Marriage et al. 2011), are accumulating, and already delivering, data. One of their goals is to use SZ cluster counts and the SZ angular correlation function as cosmological tools (Haiman et al. 2001; Weller et al. 2002; Levine et al. 2002; Majumdar \& Mohr 2004; Douspis et al. 2006). Such surveys are particularly powerful for detecting distant clusters, as was recently proven by results from Vanderlinde et al. (2010).

In this context ESA's Planck ${ }^{1}$ mission, launched on 14 May 2009 , carries a scientific payload consisting of an array of 74 detectors sensitive to a range of frequencies between roughly 25 and $1000 \mathrm{GHz}$, which scan the sky simultaneously and continuously with an angular resolution varying between about 30 arcmin (FWHM) at the lowest frequencies and about four arcmin at the highest. The array is arranged into two instruments. The detectors of the Low Frequency Instrument (LFI) are pseudo-correlation radiometers covering three bands centred at 30, 44, and $70 \mathrm{GHz}$. The detectors of the High Frequency Instrument (Lamarre et al. 2010; Planck HFI Core Team 2011a, HFI;) are bolometers covering six bands centred at 100, 143, $217,353,545$, and $857 \mathrm{GHz}$ with bolometers cooled to $0.1 \mathrm{~K}$. The design of Planck allows it to image the whole sky approximately twice per year, with an unprecedented combination of sensitivity, angular resolution, and frequency coverage. The Planck satellite, its payload, and its performance as predicted at the time of launch are described in 13 articles included in a special issue (Volume 520) of Astronomy \& Astrophysics. The

\footnotetext{
1 Planck (http://www.rssd.esa.int/Planck) is a project of the European Space Agency (ESA) with instruments provided by two scientific consortia funded by ESA member states (in particular the lead countries France and Italy), with contributions from NASA (USA) and telescope reflectors provided by a collaboration between ESA and a scientific consortium led and funded by Denmark.
} 


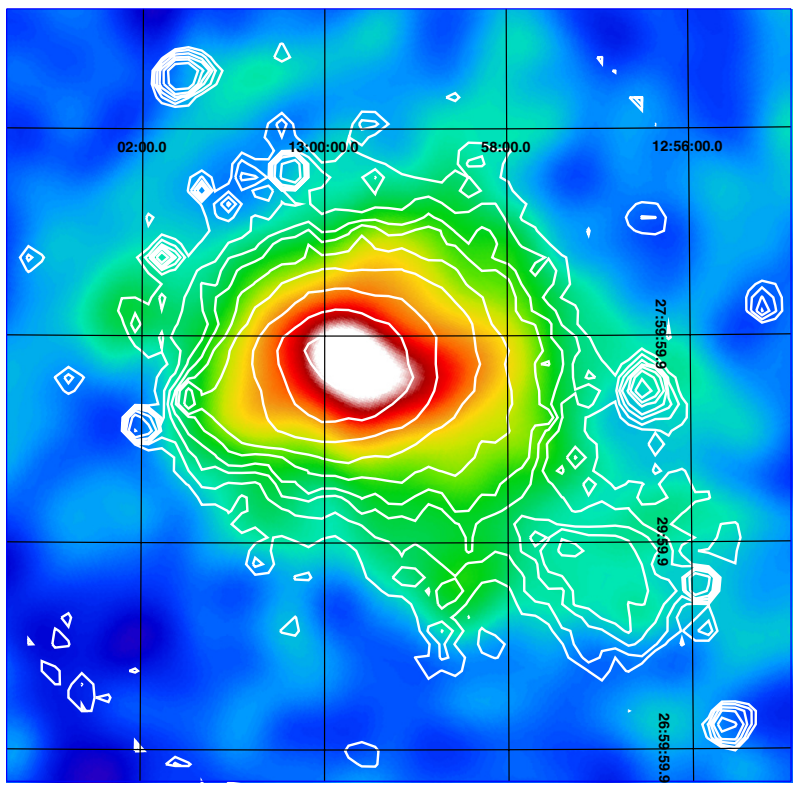

Fig. 1. Planck $y$-map of Coma on a $\sim 3^{\circ} \times 3^{\circ}$ patch with the ROSAT$P S P C$ iso-luminosity contours overlaid.

main objective of Planck is to measure the spatial anisotropies of the temperature of the CMB with an accuracy set by fundamental astrophysical limits. Its level of performance will enable Planck to extract essentially all the information in the CMB temperature anisotropies. Planck will also measure to high accuracy the polarisation of the CMB anisotropies, which not only encodes a wealth of cosmological information but also provides a unique probe of the thermal history of the Universe during the time when the first stars and galaxies formed. In addition, the Planck sky surveys will produce a wealth of information on the dust and gas in our own galaxy and on the properties of extragalactic sources.

Planck was specifically designed from the beginning to measure the SZ effect (Aghanim et al. 1997) and provide us with an all-sky SZ cluster catalogue. The first galaxy cluster searched for in the HFI data, Abell 2163 (Figs. 5 and 6), was indeed found from $100 \mathrm{GHz}$ to $353 \mathrm{GHz}$ shortly after the First Light Survey (FLS) was performed and observations in routine mode by Planck started. Three other known clusters falling in the FLS region were seen across the positive and negative parts of the SZ spectrum. The scanning strategy soon allowed us to map extended clusters such as Coma on wide patches of the sky (Fig. 1). SZ detection techniques were then applied to the data and the first blind detections were performed.

The Planck all-sky SZ cluster catalogue, with clusters out to redshifts $z \sim 1$, that will be delivered to the community at the end of the mission will be the first all-sky cluster survey since the ROSAT All-Sky Survey (RASS), which was at much lower depth (the median redshift of the NORAS/REFLEX cluster catalogue is $z \simeq 0.1$ ). Thanks to its all-sky nature, Planck will detect the rarest clusters, i.e., the most massive clusters in the exponential tail of the mass function which are the best clusters for cosmological studies. The Planck early SZ (ESZ) sample is delivered alongside the Early Release Compact Source Catalogue (ERCSC) (Planck Collaboration 2011c), the nine-band source catalogue, and the Early Cold Core (ECC) catalogue (Planck Collaboration 2011s) at http: //www.rssd.esa.int/Planck (Planck Collaboration 2011v). The ESZ is a high-reliability sample of 189 SZ clusters or candidates detected over the whole sky from the first ten months of the Planck survey of the sky.

The present article details the process by which Planck ESZ sample was constructed and validated. The Planck data and the specific SZ extraction methods used to detect the SZ candidates are presented in Sects. 2 and 3. Planck's measurements provide an estimate of the integrated Compton parameter, $Y$, of detected SZ cluster "candidates". A subsequent validation process is needed to identify which among the candidates are previously known clusters, and an additional follow-up programme is required to scientifically exploit Planck cluster data. This includes cluster confirmation (catalogue validation) and the measurement of relevant physical parameters. These different steps of the ESZ construction and validation are presented in Sect. 4 and the subsequent results are given in Sect. 5. Finally, Sects. 6-8 present the general properties of the ESZ cluster sample. Planck early results on clusters of galaxies are presented here and in a set of accompanying articles (Planck Collaboration 2011e,f,g,h).

Throughout the article, and in all the above cited Planck SZ early result papers, the adopted cosmological model is a $\Lambda \mathrm{CDM}$ cosmology with Hubble constant, $H_{0}=70 \mathrm{~km} \mathrm{~s}^{-1} \mathrm{Mpc}^{-1}$, matter density parameter $\Omega_{\mathrm{m}}=0.3$ and dark energy density parameter $\Omega_{\Lambda}=0.7$. The quantity $E(z)$ is the ratio of the Hubble constant at redshift $z$ to its present value, $H_{0}$, i.e., $E^{2}(z)=$ $\Omega_{\mathrm{m}}(1+z)^{3}+\Omega_{\Lambda}$.

\section{Planck data description}

The ESZ sample was constructed out of the Planck channel maps of the HFI instrument, as described in detail in Planck HFI Core Team (2011b). These maps correspond to the observations of the temperature in the first ten months of the survey by Planck, which give complete sky coverage. Raw data were first processed to produce cleaned time-lines (time-ordered information, TOI) and associated flags correcting for different systematic effects. This includes a low-pass filter, glitch treatment, conversion to units of absorbed power, and a decorrelation of thermal stage fluctuations. For cluster detection, and more generally for source detection, one data flag of special importance is associated with solar system objects (SSO). These objects were identified in TOI data using the publicly-available Horizon ephemeris, and the SSO flag was created to ensure that they are not projected onto the sky, in order to avoid possible false detections, ringing, etc.

Focal-plane reconstruction and beam-shape estimates were obtained using observations of Mars. Beams are described by an elliptical Gaussian parameterisation leading to FWHM $\theta_{\mathrm{S}}$ given in Planck HFI Core Team (2011b). The attitude of the satellite as a function of time is provided by the two star trackers installed on the Planck spacecraft. The pointing for each bolometer was computed by combining the attitude with the location of the bolometer in the focal plane reconstructed from Mars observations.

From the cleaned TOI and the pointing, channel maps have been made by co-adding bolometers at a given frequency. The path from TOI to maps in the HFI data processing is schematically divided into three steps: ring-making, destriping, and mapmaking. The first step averages circles within a pointing period to make rings with higher signal-to-noise $(S / N)$ ratio, taking advantage of the redundancy of observations provided by the Planck scanning strategy. The low amplitude $1 / f$ component is accounted for in the second step using a destriping technique. Finally, cleaned maps are produced using a simple co-addition 
of the Healpix-based rings ${ }^{2}$. SSO flag channel maps, used in the internal validation of the ESZ sample, were also made following the same procedure.

The noise in the channel maps is essentially white with a standard deviation of $1.6,0.9,1.4,5.0,70,1180 \mu \mathrm{K}_{\text {degree }}^{3}$ from low to high frequencies (Planck HFI Core Team 2011b). Photometric calibration is performed for the lower frequency channels at the ring level using the CMB dipole (from WMAP Hinshaw et al. 2009), and at the map level using FIRAS data (Fixsen et al. 1994) for the higher frequency channels at 545 and $857 \mathrm{GHz}$. The absolute gain calibration of HFI Planck maps is known to better than 2\% (Planck HFI Core Team 2011b).

\section{Detection and cluster extraction}

In order to generate a cluster candidate list, a suitable extraction algorithm must be run on the maps. SZ clusters can be considered as compact sources with respect to the Planck beam, but they are definitely not point sources. Their extension thus merits a special adapted processing. For this reason, several extraction methods were developed within the Planck collaboration, and those were tested and compared using the Planck Sky Model Simulation $\left(\mathrm{PSM}^{4}\right)$. The details of the comparison of the cluster extraction algorithms, called the "SZ challenge", can be found in Melin et al. (in prep.).

Methods fall into two classes: "direct" methods use individual channel maps to extract the clusters, while "indirect" methods use sky $y$-maps obtained via component separation algorithms. The methods used in this article are direct methods, with the reference method chosen on the basis of the SZ challenge. The direct detection algorithms used to construct and validate the ESZ sample incorporate prior assumptions on the cluster signal, specifically its spectral and spatial (i.e., the shape of ICM pressure profile) characteristics (see Sect. 3.1). This enhances the cluster contrast over a set of observations containing contaminating signals.

Most of the methods developed prior to the launch were applied to the Planck data, but only direct methods were favoured for implementation in the pipeline infrastructure. The following three were used to construct and validate the ESZ sample:

- a matched multi-frequency filter (MMF) algorithm, referred to henceforth as MMF3, was the reference method used for the blind detection of SZ candidates, and the construction of the ESZ list;

- two other methods (Sects. 3.3.2 and 3.3.1) were used to confirm the blind detections of the ESZ candidates.

In addition, a slightly different version of MMF3 was run as part of ESZ validation, in order to re-extract the Compton $Y$ parameter of the SZ clusters incorporating fixed cluster sizes and positions taken from X-ray observations (see Sect. 6.2).

\subsection{Baseline cluster model}

The ICM pressure profile has historically been described by an isothermal $\beta$-model (Cavaliere \& Fusco-Femiano 1978; Grego et al. 2001; Reese et al. 2002, e.g.,). However, recent X-ray observations have shown that a $\beta$-model is a poor description of

\footnotetext{
2 http://healpix.jpl.nasa.gov/ (Górski et al. 2005).

${ }^{3}$ In the following and unless otherwise stated, $\mu \mathrm{K}$ refers to equivalent CMB temperature fluctuations in $\mu \mathrm{K}$.

4 "Planck Sky Model", http://www.apc.univ-paris7.fr/ APC_CS/Recherche/Adamis/PSM/psky-en.php
}

the gas distribution in clusters, leading several authors to propose more realistic analytical functions based on a Generalised Navarro-Frenk-and-White (GNFW) profile (Nagai et al. 2007; Arnaud et al. 2010).

The baseline pressure profile used in the present work is the standard "universal" pressure profile derived by Arnaud et al. (2010). It is constructed by combining the observed X-ray pressure profile within $R_{500}$, from 31 galaxy clusters of the REXCESS sample (Böhringer et al. 2007), with data from stateof-the-art numerical simulations (Borgani et al. 2004; Nagai et al. 2007; Piffaretti \& Valdarnini 2008) out to $5 R_{500}$. In the following, $R_{500}$ is the cluster size defined as the radius where the mean enclosed density is 500 times the critical density. It relates to the characteristic cluster scale $R_{\mathrm{S}}$ through the NFW concentration parameter $c_{500}\left(R_{\mathrm{S}}=R_{500} / c_{500}\right)$.

The pressure profile model used in the present article is equivalent to the standard self-similar case described in Appendix B of Arnaud et al. (2010) ${ }^{5}$. It is equivalent to a shape function characterised by two free parameters, a central value and a characteristic scale $\theta_{\mathrm{s}}$.

The SZ effect from the hot ICM is due to the first-order correction for energy transfer in Thomson scattering. There is a spectral distortion, energy being transferred from photons in the Rayleigh-Jeans tail of the cosmic blackbody radiation to the Wien tail. In the non-relativistic limit the frequency dependence of the distortion is universal (the same for all clusters), characterised by a distinct frequency, $v \sim 220 \mathrm{GHz}$, where the TSZ effect vanishes. Below this frequency there is a decrement of the $\mathrm{CMB}$ intensity, giving an apparent decrease in the sky brightness, and above lies an enhancement.

The magnitude of the SZ effect, known as the Compton parameter $y$, depends only on the cluster's characteristics, electronic temperature $T_{\mathrm{e}}$ and density $n_{\mathrm{e}}$, as

$y=\frac{k \sigma_{\mathrm{T}}}{m_{\mathrm{e}} c^{2}} \int^{l} T_{\mathrm{e}}(l) n_{\mathrm{e}}(l) \mathrm{d} l$

where $k$ is the Boltzmann constant, $\sigma_{\mathrm{T}}$ the Thomson cross section, $m_{\mathrm{e}} c^{2}$ the electron rest mass and $l$ is the distance along the line of sight. The total SZ signal is characterised by the integrated Compton parameter denoted $Y=\int y \mathrm{~d} \Omega$, where $\Omega$ is solid angle. It can be written as $D_{\mathrm{A}}^{2} Y=\left(\sigma_{\mathrm{T}} / m_{\mathrm{e}} c^{2}\right) \int P d V$, where $D_{\mathrm{A}}$ is the angular-diameter distance to the system and $P=n_{\mathrm{e}} k T_{\mathrm{e}}$ the electron pressure. In the following, the integral performed over the sphere of radius $R_{500}\left(5 R_{500}\right)$ is denoted $Y_{500}\left(Y_{5 R 500}\right)$.Thus, as defined here, $Y_{500}$ and $Y_{5 R 500}$ have units of solid angle, e.g., $\operatorname{arcmin}^{2}$.

\subsection{Reference extraction method (matched multi-filter, MMF3)}

The ESZ sample is the result of a blind multi-frequency search in the all-sky Planck-HFI maps, i.e., no prior positional information on detected known clusters was used as input to the detection algorithm. The ESZ sample is produced by running the MMF3 algorithm, which is an all-sky extension of the matched multi-frequency filter algorithm described in Melin et al. (2006), over the six HFI frequency maps. The spectral distortion of the CMB due to the ICM can in principle be detected down to the lowest frequencies at which Planck operates; however, the beam at the lowest frequencies is large compared to typical cluster sizes. Since clusters at moderate redshifts typically span angular

\footnotetext{
5 More details on the pressure profile can be found in Planck Collaboration (2011f).
} 
scales of $\sim 5$ arcmin, the large beam of Planck at the LFI bands results in beam dilution of the SZ signal. The inclusion of the lowest Planck frequencies using the current algorithm therefore results in a lower $S / N$ for the detected sources than if only the HFI bands were used. This reduces the efficiency of SZ cluster detection, which can potentially be improved in the future with refinements to the algorithm. As a consequence, for the generation of the ESZ list, only the Planck all-sky maps at frequencies of $100 \mathrm{GHz}$ and above are considered.

The MMF algorithm, studied extensively by Herranz et al. (2002) and Melin et al. (2006), enhances the contrast, and thus $S / N$, of objects of known shape and known spectral emission profile over a set of observations containing contaminating signals. In its application for SZ, the method makes use of the universal frequency dependence of the thermal SZ effect. The filter optimises the detectability using a linear combination of maps (which requires an estimate of the statistics of the contamination) and uses spatial filtering to suppress both foregrounds and noise (making use of the prior knowledge of the cluster profile). The filter optimises cluster detection but it is not immune to contamination by false, non-SZ, detections which calls for an extensive validation procedure described in Sect. 4.

MMF3 first divides the all-sky maps into a set of 504 overlapping square patches of area $10 \times 10$ square degrees. Holes in the maps due to unsampled or badly sampled pixels are identified to construct an effective detection mask and are then filled in with the median value of the adjacent pixels. The matched multifrequency filter then combines optimally the six frequencies of each patch assuming the SZ frequency spectrum and using the reference pressure profile presented in Sect. 3.1.

Auto- and cross-power spectra used by MMF3 are directly estimated from the data and are adapted to the local instrumental noise and astrophysical contamination. For each patch, the position and the scale radius (chosen to be $5 R_{500}$ ) of the cluster profile, i.e., the cluster size $5 \theta_{500}$, are varied to maximise the $S / N$ of each detection. The algorithm hence assigns to each detected source a position, an estimated cluster size, $5 \theta_{500}$, and an integrated Compton parameter, $Y_{5 R 500}$. In the present article and unless otherwise stated the measured integrated Compton parameter, noted $Y_{5 R 500}$, is thus computed by integrating the GNFW profile within a sphere of $5 R_{500}{ }^{6}$ encompassing most of the SZ signal. The detected sources extracted from the individual patches, with their assigned sizes and integrated Compton parameters, are finally merged into an all-sky cluster list. In practice the MMF3 algorithm is run in an iterative way; after a first detection of the SZ candidates, consecutive runs centred on the positions of the candidates refine the estimated $S / N$ and candidate properties. At this stage, the uncertainty on $Y_{5 R 500}$ is provided and takes into account the uncertainty in the cluster size estimate. The MMF3 algorithm can also be performed with fixed cluster size and position to estimate the SZ signal. This version of the algorithm was used to measure the integrated Compton parameters of known X-ray clusters in the ESZ sample, as explained in Sect. 6.2.

In order to address contamination by point sources, MMF3 uses a built-in source detection algorithm to reject point sources with $S / N$ above ten which are then masked. This step avoids most of the false SZ detections associated with point sources. However, some residual contamination by non-SZ sources captured by the MMF3 algorithm may still be present and requires additional validation of the detection candidates (see Sect. 4).

\footnotetext{
${ }^{6}$ In the spherical assumption with this profile, $Y_{500}$ the integrated Compton parameter within $R_{500}$ relates to $Y_{5 R 500}$ by $Y_{5 R 500}=1.81 \times Y_{500}$.
}

\subsection{Other extraction methods}

The two other "direct" SZ detection methods used to confirm the blind detections of the ESZ candidates by MMF3 are discussed below. These methods previously compared rather well to each other within the SZ challenge match in terms of the detection properties (especially for high $S / N$ sources). Their estimated sizes and SZ signals agree on average as well, though they differ on a case by case basis.

\subsubsection{The matched multi-filter method, MMF1}

The MMF1 algorithm is a completely independent implementation of the multi-frequency matched filter integrated within the Planck-HFI pipeline and infrastructure. A more detailed description of MMF1 is given in Melin et al. (2011). The full-sky Planck frequency maps are divided into 640 flat patches, each 14.66 degrees on a side (corresponding to 512 by 512 pixels), with overlapping regions of six degrees. The performance of the MMF algorithm is extremely sensitive to the quality of the estimated auto- and cross-power spectra of the background component in each frequency map. The size of the patches thus needs to be large enough to ensure a representative assessment of the background. The large overlap between patches was chosen so that all detections in a two-degree border around the edge of the patch may be discarded.

The detection of the SZ-candidates is performed on all the patches, and the resultant sub-catalogues are merged together to produce a single SZ-candidate catalogue. Similarly to MMF3, the candidate size is estimated by filtering the patches over the range of potential scales, from point-source sized objects and larger, and finding the scale which maximises the $S / N$ of the detection of the candidate. In the version used on the Planck data, when merging sub-catalogues produced from the analysis of individual patches, it is also the $S / N$ of the detection which is used when deciding which detection of the candidate is kept.

\subsubsection{PowellSnakes (PwS) for SZ}

PowellSnakes (PwS) is quite different from the MMF methods. It is a fast Bayesian multi-frequency detection algorithm designed to identify and characterise compact objects buried in a diffuse background. The detection process is grounded in a statistical model comparison test where two competing hypotheses are compared: the detection hypothesis and the null hypothesis. The statistical foundations of PwS are described in Carvalho et al. (2009).

Similarly to the MMF algorithms, a template parameterised SZ pressure profile is assumed known and representative of the majority of the cluster population observable with the resolution and noise characteristics of the instrumental setup. According to our data model, the pixel intensities result from the contribution of three independent components: the SZ signal, the astronomical background component, and the instrumental pixel noise. The last is assumed to be a realisation of a homogeneous stationary Gaussian random white noise process. The background astronomical components and the pixel noise are assumed uncorrelated and can each be modelled locally by a homogeneous Gaussian process.

The algorithm starts by minimising the model's likelihood ratio with respect to the model's parameters by using a Powell minimiser iteratively one source at a time. We assume that the sources are well separated and the fields not too crowded. The parameter estimation and the acceptance/rejection threshold is 
defined using Bayesian approach with priors adjusted on the Planck Sky Model SZ Catalogue.

PwS performs on flat $512 \times 512$ pixel patches of 14.66 degrees on a side. When applying a Galactic cut of $|b|>14$ degrees, PwS splits the sphere into 2324 patches. However, only detections lying inside the inner $256 \times 256$ pixels are considered. So, on average PwS detects each cluster more than three times (usually four times), increasing the reliability of the detection. The selection of the candidate detection that goes into the final catalogue uses the Bayesian mode of PwS, based on the highest ratio of model posteriors.

\section{Validation of the ESZ sample}

The SZ validation process, Fig. 2, is an integrated HFI-LFI effort within Planck Working Group 5 (WG5 ${ }^{7}$ ) "Clusters and Secondary anisotropies". It has been established in order to validate the full SZ candidate lists obtained from the extraction methods developed by the Planck collaboration. It relies mainly on a three-stage process detailed in the following subsections:

\section{- Internal validation steps based on Planck data:}

- search for and rejection of associations with SSOs and artefacts;

- rejection of sources with rising spectral energy distribution in the high HFI frequency bands;

- cross-check with other Planck source catalogues to reject SZ candidates identified with cold cores (CC) and other Galactic sources; and

- redundant detections of the same candidates by methods other than the reference one.

- Candidate identification steps based on ancillary data:

- identification of SZ candidates with known clusters from existing X-ray, optical/near infrared (NIR), and SZ catalogues and lists; and

- search in NED and SIMBAD databases.

- Follow-up programmes for verification and confirmation of SZ candidates.

\subsection{Construction of ESZ sample and internal validation}

The construction of the ESZ list of SZ candidates starts with the blind detection of candidates using the implementation of the MMF3 algorithm at the US Planck Data Center applied to Planck-HFI channel maps at Galactic latitudes $|b|>14 \mathrm{deg}$. A total of about 1000 blind SZ candidates are detected with $S / N \geq$ 4. As discussed above, the MMF3 algorithm uses prior information on the SZ spectrum and on the cluster shape. However, especially due to the beam-sizes of the order of a few arcminutes, the resulting list of SZ candidates is not immune from false detection due mainly to dust emission at high frequencies from the interstellar medium (ISM) or infrared sources, and very moderately to the CMB fluctuations at low frequencies (see illustrations of channel maps in upper panels of Fig. 5). In the following, we do not explicitly check for association with extragalactic point sources emitting at Planck-HFI frequencies, which is essentially dealt with internally by the MMF3 algorithm (Sect. 3.2). Some residual contamination of the SZ Compton $Y$ parameter by point sources may, however, still be present (see Sect. 6.4 for a specific discussion).

\footnotetext{
7 http://www.ita.uni-heidelberg.de/collaborations/ planck/
}

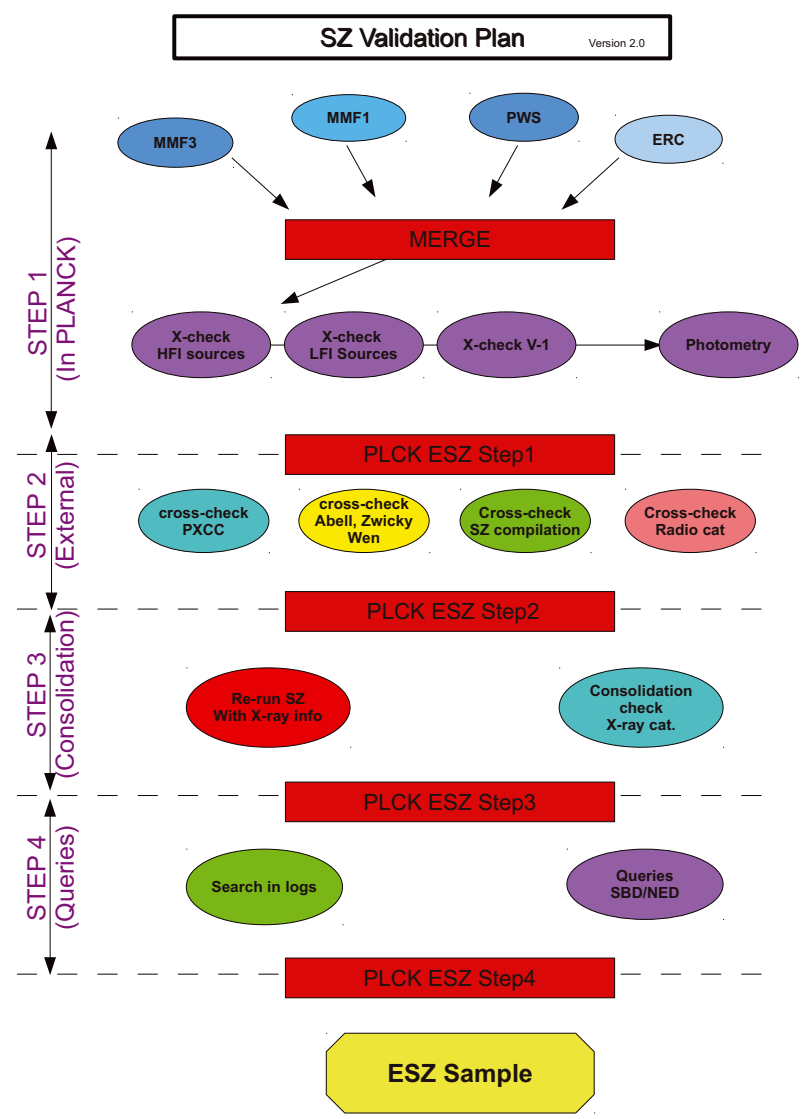

Fig. 2. Flowchart of the SZ validation process applied to the Planck cluster sample.

The internal validation process starts by removing spurious detections from the output list of blind SZ candidates, which is achieved in two steps. We first reject the candidates showing rising spectral energy distributions in the highest Planck-HFI frequency bands. They represent around $14 \%$ of the initial blind SZ candidates. Second, the remaining blind SZ candidate sample is further cleaned by rejecting all objects associated with either Galactic sources, or CC detected using the CoCoCodet algorithm (Montier et al. 2010; Planck Collaboration 2011s) within a 7 arcmin radius of $\mathrm{SZ}$ candidates. This step further reduces the sample of remaining SZ candidates by about $17 \%$.

After this two-step process, the initial blind SZ candidate sample has been reduced to around 770 blind SZ candidates with $S / N \geq 4$. However at this $S / N$ level many candidates will not correspond to actual clusters. Theoretical predictions based on the PSM simulations indicate that the purity (ratio of true to all detections) is expected to be of the order of $70 \%$ at $S / N=4$ (Fig. 15). The simulations do not account fully for the complexity of the true sky nor for the inhomogeneity of the noise across the sky. The actual purity is thus likely to be worse than the prediction. In order to ensure a high level of purity in the ESZ sample and based on lessons learnt from the XMM-Newton observations of low $S / N$ candidates (see Planck Collaboration 2011e), an early decision was made to cut at a higher $S / N$ level of $S / N \geq 6$ for this first Planck data release. This more stringent condition retains $201 \mathrm{SZ}$ cluster candidates. Taking advantage of the outcome of the follow-up programme for cluster confirmation by XMM-Newton, we further retain only the SZ candidates detected blindly by the MMF3 algorithm and at least one other method, be it MMF1 or PowellSnakes. This results in 190 SZ cluster candidates; these constitute the baseline ESZ sample. A 
detailed inspection of the SZ maps and spectra of the 11 discarded SZ candidates was performed (see Sect. 5.2.2) and confirmed that these sources were false detections.

A final internal check consisted of searching for associations of the obtained $190 \mathrm{SZ}$ candidates with possible artefacts such as low-frequency noise stripes, ringing from neighbouring bright sources, hot pixels, non-observed pixels or poorly sampled pixels in the vicinity of SSO-flagged regions. None of the 190 ESZ candidates was associated with such artefacts.

\subsection{Candidate identification with ancillary data}

The second stage of the SZ validation process consisted of crossmatching the obtained list of 190 blind SZ candidates with external cluster catalogues in X-rays, optical and SZ domains. This allowed us to identify the SZ candidates associated with previously known clusters and consequently isolate the Planck candidate new clusters.

\subsubsection{With X-ray cluster catalogues}

For the association of Planck SZ candidates from the blind extraction with known X-ray clusters, we have used the MetaCatalogue of X-ray detected Clusters of galaxies (MCXC, Piffaretti et al. 2011). This homogenised compilation of X-ray detected clusters of galaxies comprises approximately 1800 clusters from publicly available ROSAT All Sky Surveybased (NORAS, REFLEX, BCS, SGP, NEP, MACS, and CIZA) and serendipitous (160SD, 400SD, SHARC, WARPS, EMSS, etc.) cluster catalogues.

For each X-ray cluster in the MCXC several properties are available, amongst which are the X-ray centroid coordinates, redshift, identifiers, and $L_{500}{ }^{8}$. The luminosities are adopted as proxies to estimate the total mass $M_{500}$ using the $L-M$ relation from REXCESS (Pratt et al. 2009), and radius $R_{500}$, and to predict the integrated Compton $Y_{5 R 500}^{L_{X}}$, or alternatively $Y_{500}^{L_{X}}$, as detailed in Planck Collaboration (2011f) as well as other Planckrelated quantities.

Because the MCXC compilation includes only clusters with available luminosity (redshift) information, we supplement it with about 150 clusters where this information is missing. This implies that for the latter only centroid positions are available. The resulting meta-catalogue, for simplicity referred to as MCXC in the reminder of the article, is extensively used during the external validation process. For a given Planck candidate cluster we identify the closest MCXC cluster. The reliability of the association is checked based on the distance, as compared to the cluster size, and on the measured $Y_{5 R 500}($ or $S / N)$ values, as compared to the expected values $Y_{5 R 500}^{L_{X}}($ or $S / N)$ for the MCXC clusters.

\subsubsection{With optical cluster catalogues}

The baseline for the identification of blind SZ candidates from the ESZ with clusters known in the optical is the cross-match with the Abell cluster catalogue (Abell 1958, 5250 clusters of which 1026 have a redshift) and the Zwicky cluster catalogue (Zwicky et al. 1961,9134 objects). The association criterion here was a positional match with a search radius for both catalogues set to five arcminutes.

\footnotetext{
8 The X-ray luminosities as measured within an aperture of radius $R_{500}$.
}

Furthermore, the ESZ sample was cross-checked against the MaxBCG (Koester et al. 2007) and Wen et al. (2009) catalogues with a search radius of 5 arcmin.

\subsubsection{With known SZ clusters}

The identification of SZ candidates is also performed at millimetre wavelengths by cross-matching the SZ candidate list with a compilation of SZ observed galaxy clusters from the literature undertaken by Douspis et al. (in prep.). This compilation is based on SZ observations conducted with the numerous experiments developed during the last 30 years (Ryle, OVRO, BIMA, MITO, Nobeyama, SZA, APEX-SZ, AMI, Diabolo, Suzie, Ryle, AMIBA, ACBAR, etc). It also includes the new clusters recently discovered through their SZ signature by ACT and SPT. In total the compilation comprises $111 \mathrm{SZ}$ clusters including 28 newly discovered by ACT and SPT (Menanteau et al. 2010; Vanderlinde et al. 2010). The association of the Planck SZ candidates was based on positional matching with a search radius of five arcminutes.

\subsubsection{Queries in SIMBAD and NED databases}

The information provided from querying databases is mainly redundant with cross-checks with cluster catalogues in X-ray or optical. However, running both cross-matches is important to avoid missing a few associations. It is also important to retrieve the information on redshifts for those identified clusters not included in the MCXC. We therefore performed a systematic query in SIMBAD. The adopted search radius was set to five arcminutes. For NED, no systematic query was implemented. Cluster candidates within the same search radius were rather checked against a list of objects retrieved from NED flagged as "Clusters of galaxies". Finally the candidates were also checked against the X-ray cluster database (Sadat et al. 2004, BAX:).

\subsection{Follow-up programme for validation and confirmation}

In parallel to the effort of SZ candidate cross-identification, a coherent follow-up programme targeted towards the verification/validation of the cluster candidates in the SZ catalogue was put into place in the form of an internal roadmap. The main goals of this follow-up programme are to confirm Planck candidates as new clusters, and as a consequence to better understand both the SZ selection criteria in the Planck survey and the reliability of selected sources.

Considering the complementarity of X-ray, optical and IR/SZ, observational follow-ups have been coordinated to optimise the validation and the understanding of the Planck selection. In practice, this took the form of a confirmation programme relying on observations with XMM-Newton ${ }^{9}$ making use of Director Discretionary Time (DDT) as detailed in Planck Collaboration (2011e). This is complemented by observations in the optical using the European Northern Observatory facilities (ENO), the European Southern Observatory $2.2 \mathrm{~m}$ telescope, and two pilot programmes, one with the WISE experiment (Wright et al. 2010) for the search of overdensities in

${ }^{9}$ XMM-Newton is an ESA science mission with instruments and contributions directly funded by ESA Member States and the USA (NASA). 

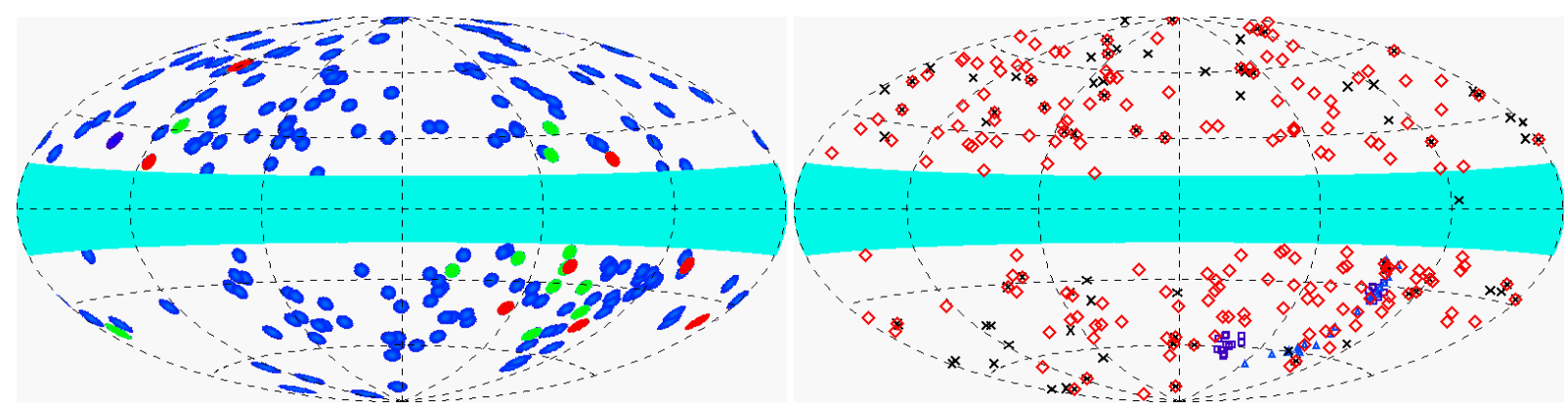

Fig. 3. Distribution of ESZ clusters and candidate clusters on the sky (Galactic Aitoff projection). Left panel: in blue are ESZ clusters identified with known clusters, in green the ESZ confirmed candidates, and in red the ESZ candidate new clusters yet to be confirmed. Right panel: in red diamonds the ESZ sample, in black crosses the compilation of SZ observations prior to 2010, in dark blue triangles ACT clusters from Menanteau et al. (2010), and in purple squares SPT clusters from Vanderlinde et al. (2010). The blue area represents the masked area of $|b|<14$ deg.

the IR data, and one with the Arcminute MicroKelvin Imager ${ }^{10}$ (AMI, Zwart et al. (2008)) for the confirmation of Planck candidates with SZ observations.

An ensemble of SZ candidates spanning a range of $S / N$ between four and eleven was selected from earlier versions of the HFI channel maps and sent to the above-mentioned facilities. The targets were selected from a list of SZ candidates after the external validation stage (i.e., identification of known clusters). They went through visual inspection of their maps and spectra produced by all the available methods described in Sect. 5.2.2. Furthermore, in order to avoid duplicating existing observations of candidates with the same or similar facilities, the cluster candidates were further cross-matched with logs of X-ray, optical, and NIR observatories.

The search in X-ray observatories (ROSAT, Suzaku, XMM-Newton, and Chandra) was performed using the HEASARC tool ${ }^{11}$. For XMM-Newton and Chandra both master catalogues and accepted GO (Guest Observer) targets were used in the search. For Suzaku, only the master catalogue was used. In the case of optical and NIR observatories, the search was performed in the public logs of several optical/infrared observatories. In some cases, this search was completed using VO (Virtual Observatory) tools ${ }^{12}$. The checked resources were: ING Archive, UKIRT Archive, ESO Archive, HST Archive (at ESO), CFHT Archive, AAT Archive, NOAO Science Archive, Multimission Archive at STScI (MAST), Gemini Science Archive, and SMOKA (Subaru Mitaka Okayama Kiso Archive). In addition, a search in the footprint of the covered area for known surveys was performed. The searched areas considered were those of SDSS, UKIDSS, and HST (ACS-WFC) as they are described in the VO footprint service ${ }^{13}$ (Budavári et al. 2007), as well as those of SPT and ACT experiments.

The details and results of the confirmation follow-up with $X M M-N e w t o n$ are given in Planck Collaboration (2011e). A total of 25 targets were observed with short snapshot exposures (i.e., $10 \mathrm{ks}$ nominal EPN) out of which 21 were confirmed as clusters or systems of multiple extended X-ray sources (i.e., double or triple). Complying with Planck policies and following the agreement between the Planck and XMM-Newton ESA project scientist, all the data are made public with the publication of the

\footnotetext{
10 AMI is a pair of interferometer arrays located near Cambridge, UK, operating in six bands between 13.5 and $18 \mathrm{GHz}$, with sensitivity to angular scales 30 arcsec-10 arcmin.

11 http://heasarc.gsfc.nasa.gov/cgi-bin/W3Browse/ w3browse.pl

12 VO command line tools http://iraf-nvo.noao.edu/vo-cli/

${ }^{13}$ http://www. voservices.net/footprint
}

Planck early results and the Planck ERCSC. Of the 21 confirmed Planck SZ sources, 11 are found in the ESZ sample and are discussed in Sect. 5.2.1. The remaining clusters with $S / N<6$ are discussed in Planck Collaboration (2011e). One candidate cluster in the ESZ sample was confirmed by AMI and WISE. None of the targets sent for observation in the optical with the ENO telescopes met the ESZ selection criteria.

\section{Results of the validation}

In the following we will detail the outcome of the external validation of the $190 \mathrm{SZ}$ candidate clusters retained after the internal validation. We find that they are distributed between known clusters (169 in total, Fig. 3 blue) and 21 candidate new clusters. Among those 21, twelve have been confirmed (Fig. 3 yellow) and these are discussed in Sect. 5.2.1. Nine remain as candidate new clusters requiring confirmation (Fig. 3 red); they are described in Sect. 5.2.2. The further checks performed on the candidate new clusters resulted in the rejection of one of the nine candidates.

The final released ESZ list thus comprises 189 clusters or candidate new clusters. The content of the released data ${ }^{14}$ is presented in Appendix A.1. Table 1 summarises the different steps of ESZ sample construction and validation detailed in the previous sections. Figures 5 and 6 show illustrations of the raw and "cleaned" channel maps (from 100 to $545 \mathrm{GHz}$ ) as well as corresponding $y$-maps, for a few clusters, with $S / N$ ranging from the highest ones to more typical ones.

\subsection{ESZ candidates identified with known clusters}

The external validation with ancillary data identified 169 clusters in total out of the 190 candidates detected blindly. They are known X-ray or optical clusters and Planck data provide the first measure of the SZ signal for the majority of them, opening a new observational window on those already known objects.

Most of the identified SZ candidates, 162 in all, were associated with known clusters from the MCXC compilation and 158 have known redshifts (provided in the compilation), X-ray luminosities, X-ray estimated sizes $\left(\theta_{500}\right)$, etc. Moreover, as expected, a very large fraction of them (127 clusters) are at the same time identified in the optical. They are mostly Abell clusters from the ROSAT X-ray cluster catalogues.

The remaining seven identified Planck clusters were obtained from search in SIMBAD (one cluster, RXJ0748.7+5941,

14 http://www.rssd.esa.int/Planck 
Table 1. Summary of the ESZ sample construction and validation steps.

\begin{tabular}{lccc}
\hline \hline Selection & & SZ Candidates & Rejected \\
\hline & $S / N \geq 6$ and good quality flag on SZ spectrum & 201 & 11 \\
& Detected by one method only & & 1 \\
\hline Bad quality flag from visual inspection & & 189 \\
KnZ sample & & 169 \\
\hline & X-ray only & 30 \\
& Optical only & 5 \\
& NEDSimbad only & 1 \\
& X-ray + Optical & 128 \\
& X-ray + SZ & 1 \\
& SZ + Optical & 1 \\
& X-ray + Optical +SZ & 3 \\
\hline New Planck clusters & XMM confirmed & 20 \\
& AMI confirmed & 11 \\
& Candidate new clusters & 1 \\
\hline
\end{tabular}

observed by ROSAT but not part of the NORAS catalogue (Appenzeller et al. 1998) and without published redshift), from logs of observatories (one cluster, H1821+643 at $z=0.299$ (Schneider et al. 1992)) and from optical only, i.e., without an X-ray counterpart, identification with Abell or Zwicky clusters (five clusters). These are $\mathrm{ZwCl} 2120.1+2256$, AC114Northern, A3716S, ZwCl1856.8+6616, and ZwCl0934.8+5216 clusters. The last two have no published redshifts. For all these clusters, redshifts when they are available are retrieved from the SIMBAD and NED databases.

The cross-match with known SZ clusters further indicates that one cluster, AS0520, is common to Planck, ACT and SPT. Additionally, five ${ }^{15}$ clusters from ACT are in common with the Planck ESZ sample, and twelve massive clusters observed by SPT (Plagge et al. 2010) are also observed by Planck and quoted in the ESZ. Finally by comparing with the SZ compilation from Douspis et al. (in prep.) we find that, in total, 41 clusters from the ESZ sample have already been observed in SZ by previous experiments. For these clusters Planck provides us with a homogeneous set of SZ measures. Moreover, out of the full ESZ sample about $80 \%$ have been observed in SZ for the first time and have a homogeneous measurement of their Compton parameter from Planck.

Out of the known clusters in the ESZ sample, a few are given in the Early Release Compact Source Catalogue (ERCSC) (Planck Collaboration 2011v) as they were detected by the source extraction techniques used to construct the ERCSC. They are 1ES 0657-55.8 (commonly known as the bullet cluster and detected blindly with an $S / N$ of 19.7), A2218, ACO S0520, CIZA J1938.3+5409, A0119, RXC J1720.1+2637, A3376, and MACS J2135.2-0102. It is worth noting that the quoted fluxes in the ERCSC are obtained using aperture photometry on the channel maps without band merging. They cannot be compared easily with the obtained integrated Compton parameters in the present article. Moreover, two of the above-listed clusters, RXC J1720.1+2637 and MACS J2135.2-0102, suffer from astrophysical contamination that may affect the computed $Y$.

\footnotetext{
15 One of the candidate new clusters confirmed by XMM-Newton appeared in publication as one of the ACT SZ optically-confirmed clusters (Menanteau et al. 2010) to be observed by Chandra, after we scheduled it for observation with XMM-Newton: PLCKESZ G262.7-40.9/ACT-CL J0438-5419. We retain it as new candidate in the following.
}

\subsection{New Planck clusters in the ESZ sample}

The ESZ sample contains 20 new clusters or candidates clusters with $S / N$ ranging from 11.5 to 6 . As mentioned above, a follow-up programme set up to help understand the selection of Planck clusters allowed us to confirm 12 clusters. Eleven were confirmed with XMM-Newton snapshot observations, while one cluster was confirmed with AMI observations and corresponds to an overdensity of galaxies in the WISE data.

\subsubsection{Confirmed ESZ cluster candidates}

The XMM-Newton observations for confirmation of SZ candidates were based on earlier versions of the channel maps and an earlier version of the data processing than that used for the ESZ construction. The 25 targets sent for observation were selected in two different campaigns, a pilot programme (exploring $S / N$ from six down to four) and a higher $S / N$ programme (above $S / N$ of 5). Among the 21 Planck cluster candidates confirmed by snapshot observation with XMM-Newton, 11 clusters have a Planck $S / N$ above six (in the present map version) and thus meet the ESZ selection criteria. Two of them were found to be double clusters on the sky. All eleven are published in the ESZ release. Together with the remaining ten clusters confirmed by XMM-Newton, all are described in Planck Collaboration (2011e). In the following we just summarise the general properties of the new confirmed clusters in the ESZ.

The eleven new clusters in the ESZ confirmed by $X M M$-Newton have $S / N$ ranging from 11.5 to 6.3 . They were found to lie below the REFLEX flux limit of $3 \times 10^{-12} \mathrm{erg} \mathrm{s}^{-1}$, except for two confirmed clusters above the limit. These clusters happen to have associations with BSC sources and to be situated above the MACS limit; however their redshifts, $z=0.27$ and $z=0.09$ are below the considered redshifts for MACS (see the detailed discussion in Planck Collaboration (2011e)). The redshifts of the new confirmed clusters were estimated directly from X-ray observations of iron emission lines, and range between $z=0.2$ and 0.44 . Only two out of the eleven confirmed new clusters have optical redshift estimates. For one new cluster (PLCKESZ G285.0-23.7), the agreement between the X-ray estimated and photometric redshifts is quite good. The second cluster, PLCKESZ G262.7-40.9, was found to be an ACT cluster, published after the scheduling of XMM-Newton observation, for which there is a discrepancy between the X-ray-estimated 


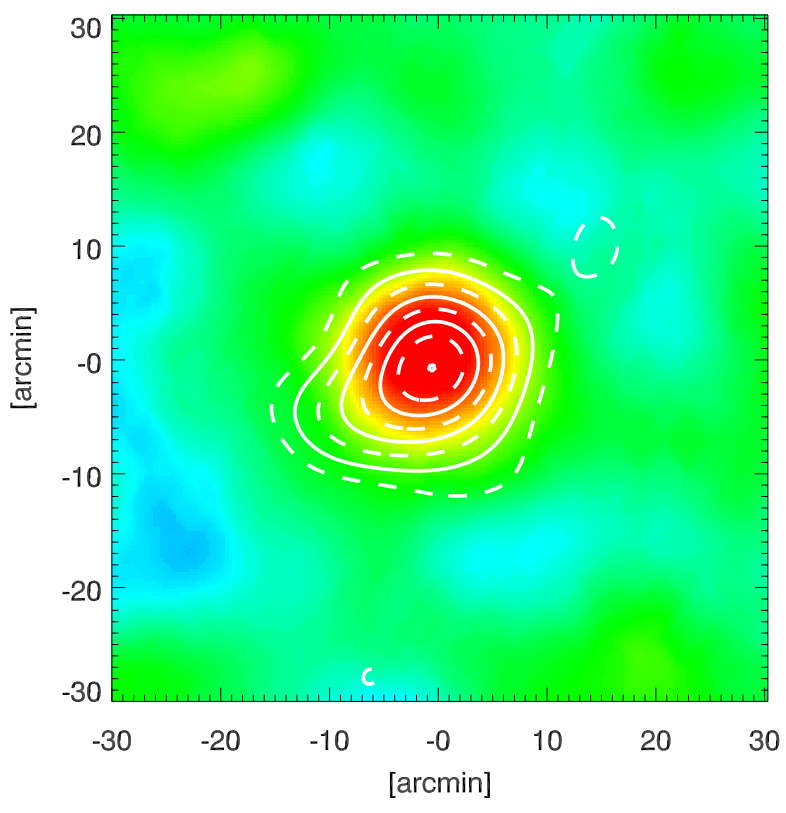

Fig. 4. $y$-map of PLCKESZ G139.59+24.19 as observed by Planck (colour image) and AMI (contours) at a common resolution of 13 arcmin. The contours are from two to nine in $S / N$ ratio.

redshift $(z=0.39)$ and the photometric redshift $(z=0.54)$ from Menanteau et al. (2010). The range in temperature spanned by the new confirmed clusters in the ESZ is from about 4 to $12 \mathrm{keV}$, and the derived masses range from about 4 to $15 \times 10^{14} M_{\odot}$. Three new clusters in the ESZ sample have masses of $10 \times 10^{14} M_{\odot}$ or above, including the most massive cluster detected by Planck with a mass of about $15 \times 10^{14} M_{\odot}$. The confirmation of the Planck new clusters by XMM-Newton provides us with positions and, most of all, a better estimate of the cluster size that will be important for the re-extraction of $Y$ values (see Sect. 6.2).

One additional candidate cluster, PLCKESZ G139.59+24.19 detected at $S / N=7.2$, was confirmed by a pilot project for confirmation with the AMI interferometer (see Fig. 4 showing the Planck $y$ map with the AMI contours, obtained after the subtraction of bright sources with the large array observations, overlaid). The Planck cluster was detected at $9 \sigma$ by AMI in a long-time exposure of approximately $30 \mathrm{~h}$. Preliminary results from AMI give an integrated Compton parameter of $Y_{5 R 500}=(17.0 \pm 1.7) \times 10^{-4} \mathrm{arcmin}^{2}$, extracted fixing the cluster size to the estimated size from Planck. The Planck value, $Y_{5 R 500}=(32 \pm 13) \times 10^{-4}$, is obtained from the blind detection of the cluster. The error bar takes into account the uncertainty in the cluster size estimate by the MMF3 algorithm. A detailed comparison is planned. This same cluster was also confirmed at a $S / N$ level of five by WISE.

\subsubsection{ESZ candidate new clusters}

A closer inspection of the ESZ candidate new clusters was performed in order to ensure the reliability of the retained candidate new clusters. The same close inspection was also performed, a posteriori, in order to confirm the rejection of the 11 candidates excluded in the final steps of the ESZ construction because they were observed solely by MMF3 (Sect. 4.1). This closer inspection of the candidates was based on both internal (using Planck alone) and external data.

For the in-depth inspection of the Planck data, we used cleaned channel maps, reconstructed $y$-maps and SZ spectra.
All these products are quite sensitive to the procedure used for cleaning the channel maps, i.e., to the component separation method. We therefore simultaneously employed different cleaning approaches developed by the Planck collaboration, briefly described below, in order to ensure convergence and redundancy in the derived conclusions. One of the methods is based on the construction of SZ $y$-maps centred on the ESZ candidate positions using the Modified Internal Linear Combination Algorithm (MILCA, Hurier et al. 2010) applied independently on each SZcentred patch. The contribution from other sources of sky emission such as thermal dust and radio and infrared sources is thus more accurately reduced. Other approaches based on local component separation and aperture photometry were also developed in order to check the $y$-maps and SZ spectra of the candidates. Patches centred on the SZ candidates are produced from the Planck channel maps and the IRIS map (Miville-Deschênes \& Lagache 2005). Local component separation is performed by decorrelating from the low-frequency channels an extrapolation of the dust emission computed with the $857 \mathrm{GHz}$ and IRIS maps. The "dust-free" $217 \mathrm{GHz}$ map is then removed from all channels and visual inspection can then be performed on these cleaned patches. From this set of maps we then obtain SZ reconstructed $y$-maps and an SZ spectrum by applying aperture photometry to each patch. The internal inspection of the Planck data ( $y$-maps, frequency maps and spectra) therefore provides us with a set of quality flags that were used for the selection of targets for the follow-up programmes and that are used for a qualitative assessment of the reliability of the candidates.

Converging negative quality assessments result in the rejection of the SZ candidates. However in most cases, it is useful to combine and complement the Planck-internal quality flags with external information. In practice this consists in searching for associations with FSC (Faint Source Catalogue) and BSC (Bright Source Catalogue) RASS sources, searching in, and visualising, the RASS maps at the candidate cluster positions, and finally performing visual checks of the DSS (Digital Sky Survey) images in the candidate field (within a five arcminute radius from the Planck position). Based the lessons learnt from the $X M M-N e w t o n$ confirmation programme, the association of candidates with FSC or BSC-RASS sources (in the five arcminute radius field) was considered as an indication of the reliability of the candidate. The presence of an excess in the count-rate RASS images in the candidate field was also used as a reliability flag. The DSS images were used simply as an "empirical" assessment of the presence of an overdense region. It is worth noting that the external information provided in particular by the RASS data never supersede the Planck-internal quality flags. As a matter of fact, two of the confirmed new clusters had neither FSC nor BSC associations. Conversely, associations with FSC and BSC-RASS sources were found for SZ candidates that turned out to be false detections (Planck Collaboration 2011e).

Using the internal quality flags and the additional external checks, out of the nine candidate new clusters retained by the ESZ construction, seven were judged reliable. Two candidate new clusters had rather poor quality flags and no external associations. One of them was found to be associated with dust cloud emission. Note that this source was not flagged by the crossmatch with the CC and Galactic sources, nor identified with a rising spectral distribution at high frequencies during the internal validation and ESZ construction. This candidate was rejected from the final ESZ sample, reducing the total number of clusters and candidate clusters from 190 to 189 . The second cluster candidate with low reliability (PLCKESZ G189.84-37.24), was kept in the ESZ list as it was not associated clearly with any non-SZ 
Planck Collaboration: Planck early results. VIII.

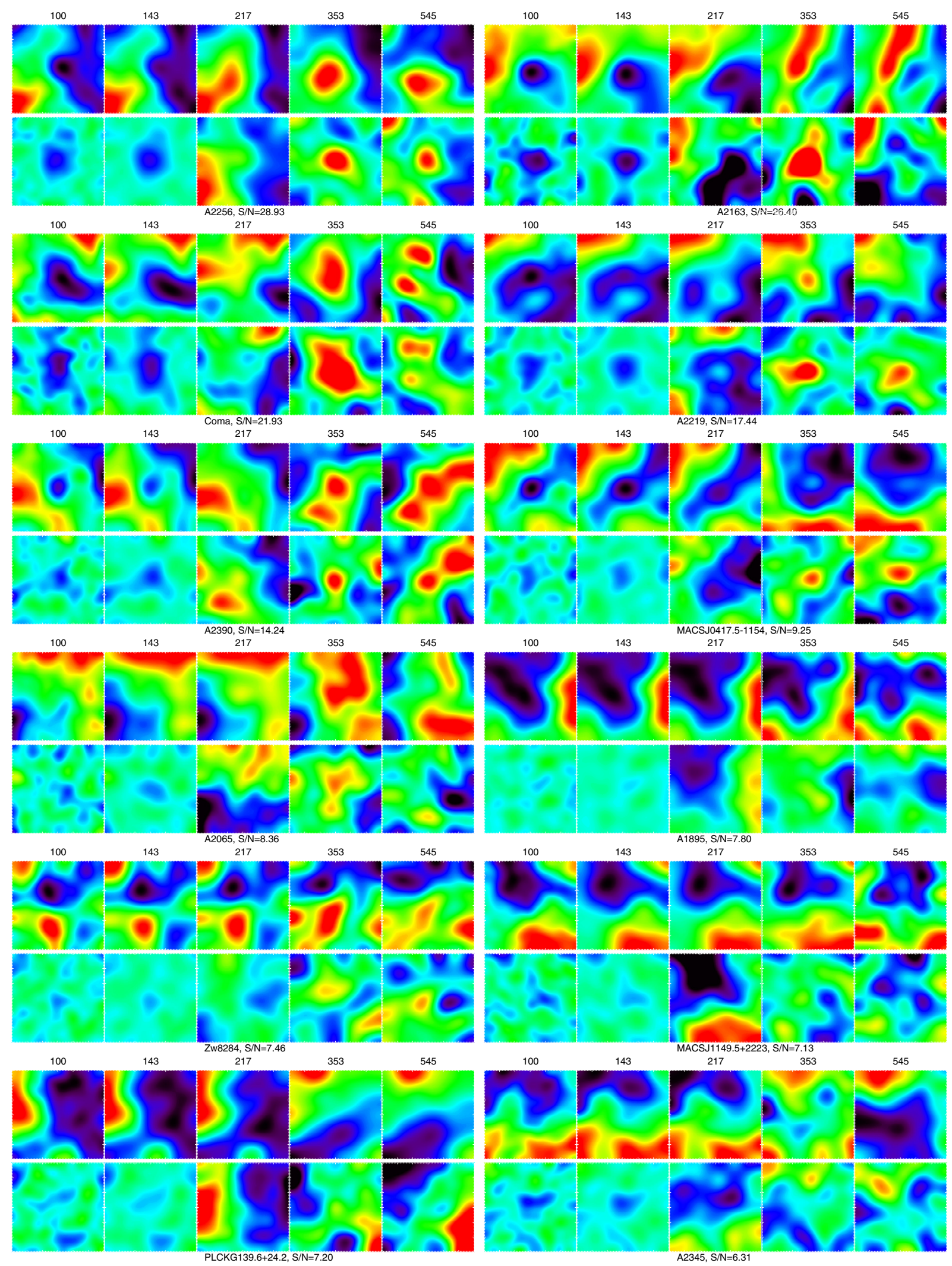

Fig. 5. Observations of a few clusters from the ESZ sample. For each cluster, the upper panels show the raw (1 square degree) maps at 100, 143, 217,353 , and $545 \mathrm{GHz}$. The lower panels show the corresponding cleaned maps (see Sect. 5.2.2). These clusters span $S / N$ from 29 to 6 from the upper left to the lower right.

source. Table 2 summarises the external information associated with the candidate new clusters in the ESZ sample ${ }^{16}$.

\footnotetext{
${ }_{16}$ During the review process 6 of the 8 Planck cluster candidates were confirmed by SPT (Story et al. 2011; Williamson et al. 2011) and AMI
}

(Hurley-Walker et al. 2011) experiments independently of the Planck collaboration. 

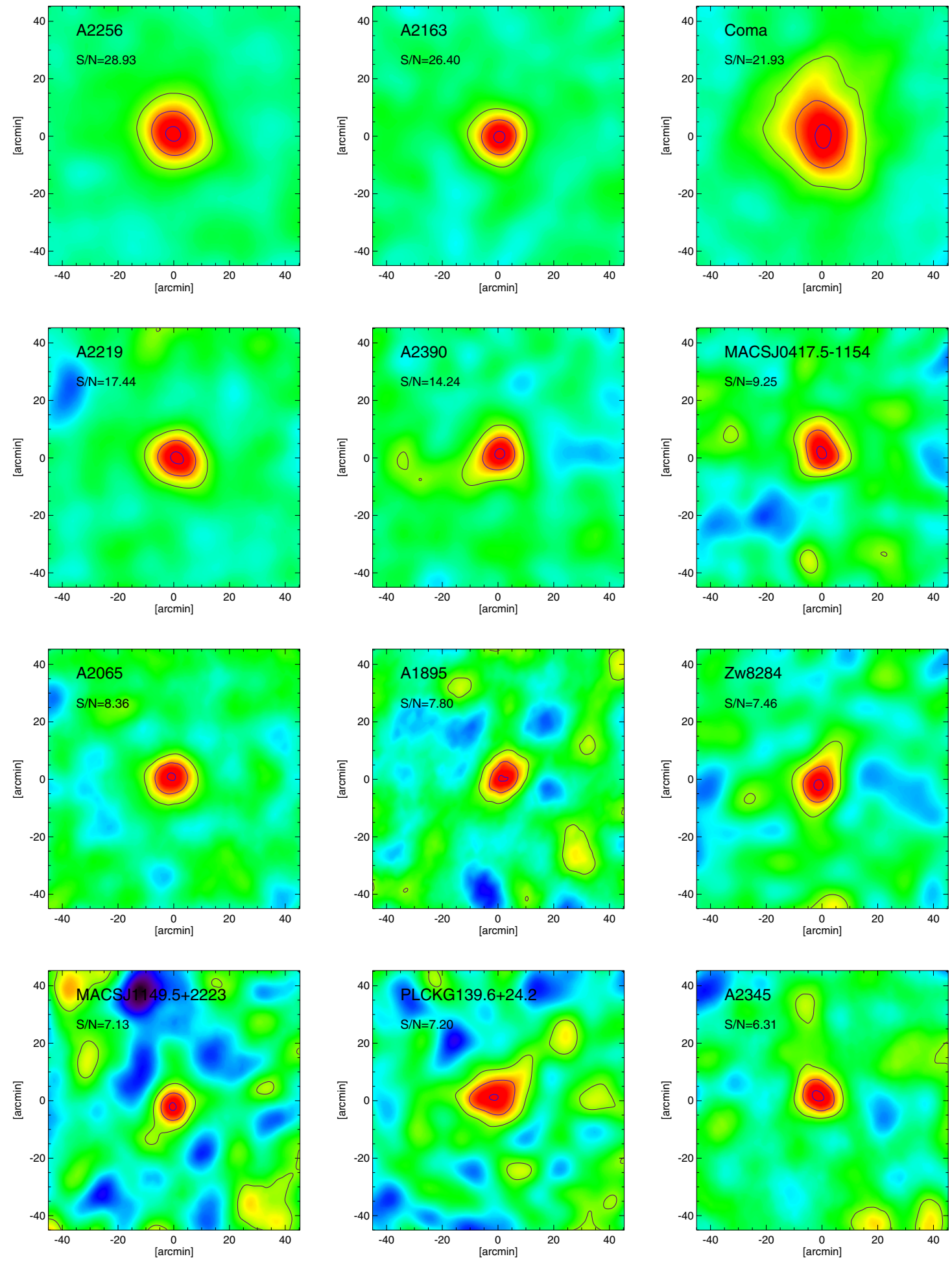

Fig. 6. Illustrations of reconstructed $y$-maps $\left(1.5^{\circ} \times 1.5^{\circ}\right.$, smoothed to $\left.13 \mathrm{arcmin}\right)$ for clusters spanning $S / N$ from 29 to 6 from the upper left to the lower right.

\section{Error budget on the cluster parameters}

\subsection{Position}

The ESZ sample contains a list of 189 clusters or candidate clusters distributed over the whole sky with positions obtained from blind detection with the MMF3 algorithm. Based on the simulation used for the SZ challenge comparison, we find that MMF3 recovers cluster positions to $\sim 2$ arcmins on average. However, there is a large scatter in the positional accuracy, as seen in Fig. 7.

For the $158 \mathrm{ESZ}$ candidates identified as X-ray clusters with known X-ray size, the coordinates of the X-ray counterpart are given by the MCXC. The X-ray position is also given for the Planck cluster candidates confirmed by XMM-Newton as single objects. The comparison of the SZ candidate positions derived from the blind detection with the X-ray positions of the identified 
Planck Collaboration: Planck early results. VIII.

Table 2. For the Planck candidate new clusters not yet confirmed at the time of submission, external information from RASS.

\begin{tabular}{|c|c|c|c|c|c|}
\hline Name & $\begin{array}{c}\text { RASS } \\
\text { association }\end{array}$ & $\begin{array}{c}\text { Distance to } \\
\text { source (arcmin) }\end{array}$ & $\begin{array}{c}S / N \text { of } \\
\text { RASS source }\end{array}$ & $\begin{array}{c}S / N \text { of RASS } \\
\text { in Planck aperture }\end{array}$ & Note \\
\hline PLCKESZ G115.71+17.52 & BSC & 0.17 & 8.7 & 8.5 & $\begin{array}{l}\text { Possible contamination by } \\
\text { dust emission }\end{array}$ \\
\hline PLCKESZ G121.11+57.01 & FSC & 1.72 & 2.9 & 4.1 & $\begin{array}{l}\text { Possible association with } \\
\text { WHL J125933.4+600409 } \\
\text { from Wen et al. (2009), } z= \\
0.33\end{array}$ \\
\hline PLCKESZ G189.84-37.24 & None & - & - & 1.3 & $\begin{array}{l}\text { Low reliability, high } \\
\text { level of contamination by } \\
\text { Galactic emission }\end{array}$ \\
\hline PLCKESZ G225.92-19.99 & FSC & 1.11 & 2.5 & 6.7 & $\begin{array}{l}\text { With XMM-Newton and } \\
\text { HST pointed observations }\end{array}$ \\
\hline PLCKESZ G255.62-46.16 & FSC & 0.9 & 2.7 & 3.8 & $\begin{array}{l}\text { With ESO and Suzaku } \\
\text { pointed observations }\end{array}$ \\
\hline PLCKESZ G264.41+19.48 & BSC & 1.22 & 4.6 & 5.7 & \\
\hline PLCKESZ G283.16-22.93 & FSC & 0.54 & 3.6 & 4.2 & \\
\hline PLCKESZ G304.84-41.42 & BSC & 0.55 & 3.6 & 5.1 & $\begin{array}{l}\text { With ESO pointed observa- } \\
\text { tions }\end{array}$ \\
\hline
\end{tabular}

or confirmed clusters for a total of 167 clusters is shown in Fig. 7 , left panel. The positional offset between Planck blind and X-ray positions, $D_{\mathrm{SZ}-\mathrm{X}}$, is of the order of 2 arcmin on average, consistent with the estimates obtained from the SZ challenge simulation. Very few clusters (8 in total over 167) have an offset $D_{\mathrm{SZ}-\mathrm{X}}>4$ arcmin, and stand out as clear outliers in the distribution. It is worth noting that such a positional offset combines both the uncertainty in the position reconstruction from MMF3 and the possible physical offset between the centroids of $\mathrm{X}$-ray and SZ signals (e.g., in merging clusters). The eight clusters with $D_{\mathrm{SZ}-\mathrm{X}}>4$ arcmin are all nearby merging clusters or members of larger structures such as A3532 in the Shapley supercluster, or contaminated by radio source emission. The cluster A1066 $(z=0.07)$, which has the largest positional offset $\left(D_{\mathrm{SZ}-\mathrm{X}}=10\right.$ arcmin), is in the Leo Sextans supercluster (Einasto et al. 2001). In addition, it may suffer from point source contamination. The cluster Abell 1367 at $z=0.02$ with $D_{\mathrm{SZ}-\mathrm{X}}=7.8$ arcmin is a young cluster currently forming at the intersection of two filaments (Cortese et al. 2004) with complex gas density and temperature structures (Ghizzardi et al. 2010).

As seen from Fig. 7, right panel, large (greater than four arcmin) offsets are only seen in nearby clusters (seven out of the eight clusters with $D_{\mathrm{SZ}-\mathrm{X}}>4$ have redshifts lower than 0.08). They remain smaller than the cluster size, as expected for offsets dominated by physical effects. On average, the offsets tend to decrease with increasing redshift and seem to become independent of redshift above $z \sim 0.3$. This is due to the decreasing contribution of possible physical offsets, which become unresolved. The overall offset, including the absolute reconstruction uncertainty, remains smaller than the cluster size for most of the clusters in the ESZ $\theta_{500}$ (Fig. 7, right panel). However, we expect that it will be of the order of cluster size for clusters at higher redshifts than the range currently probed. This positional offset is therefore an additional source of uncertainty in the cluster position which needs to be taken into account in the follow-up observations for candidate confirmation.

\subsection{Cluster size-Y degeneracy}

The MMF algorithm, and more generally algorithms that are based on the adjustment of an SZ profile to detect clusters, generally perform better than algorithms which do not assume an SZ

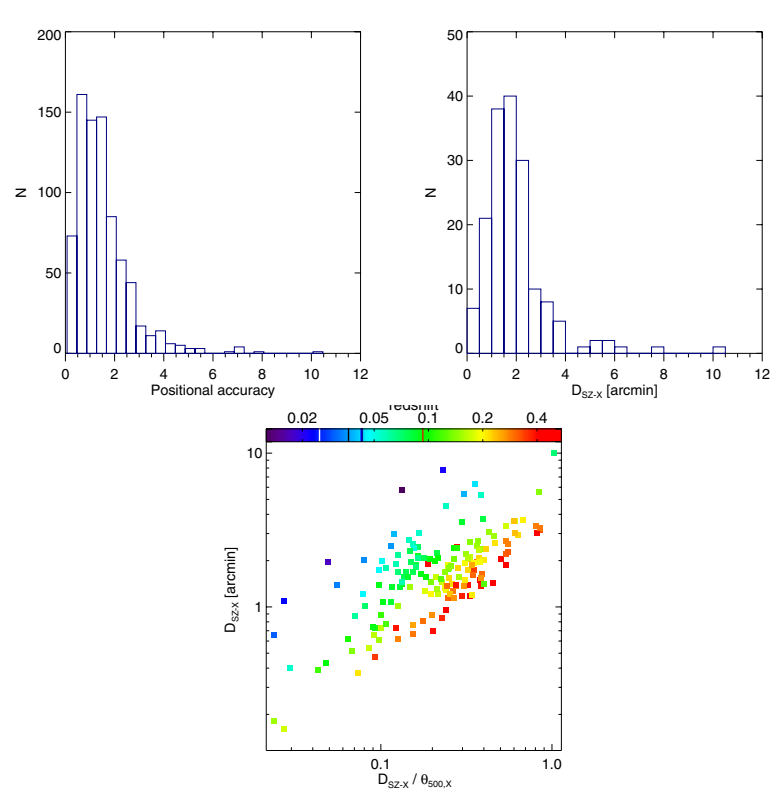

Fig. 7. Upper left panel: positional accuracy from MMF3 based on simulations for the SZ challenge. Upper right panel: distribution of the distance between the SZ blind position and the X-ray position $\left(D_{\mathrm{SZ}-\mathrm{X}}\right)$ for 167 known, or confirmed with XMM-Newton, X-ray clusters. Lower panel: separation of the SZ blind and X-ray positions $D_{\mathrm{SZ}-\mathrm{X}}$ as a function of $D_{\mathrm{SZ}-\mathrm{X}}$ normalised to the cluster size $\theta_{500, \mathrm{X}}$.

profile. The GNFW profile used in the present study corresponds to a shape function characterised by two parameters, the central value and a characteristic scale $\theta_{\mathrm{s}}$ (with $\theta_{\mathrm{s}}=\theta_{500} / c_{500}$ and $c_{500}$ is the concentration parameter). Simulations showed that the intrinsic photometric dispersion of the recovered integrated Compton parameter, with a GNFW profile, could be of order $30 \%$ (see Fig. 8) even with the prior information on the pressure profile. This is due to the difficulty of estimating the cluster size, which in turn is degenerate with the SZ $Y$ estimate.

This cluster size- $Y$ degeneracy is illustrated, here using PwS, in two extreme situations (Fig. 9) showing the likelihood plots (integrated Compton vs cluster size) of an extended high $S / N$ cluster such as Coma (blue contours) and an unresolved $S / N=6$ cluster (black contours). In both cases, the integrated Compton 


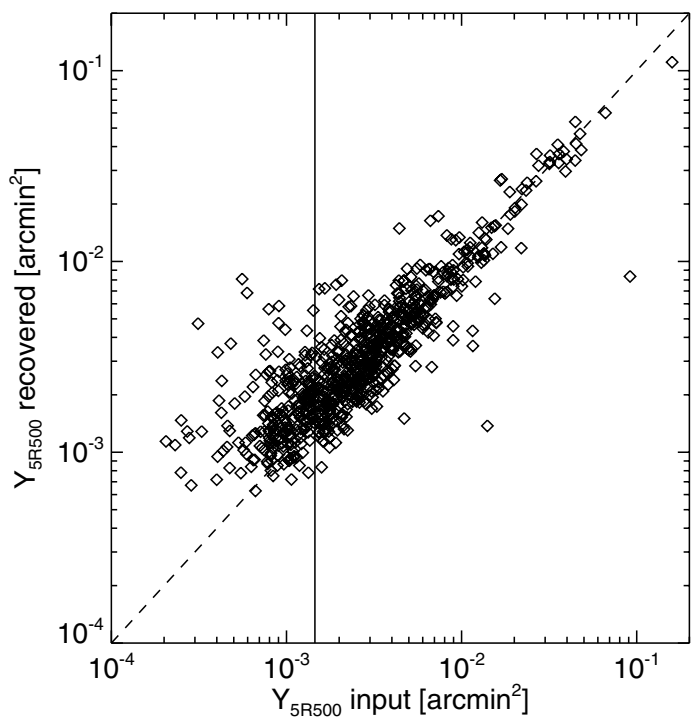

Fig. 8. Input versus recovered integrated Compton parameter from MMF3, based on simulations for the SZ challenge.

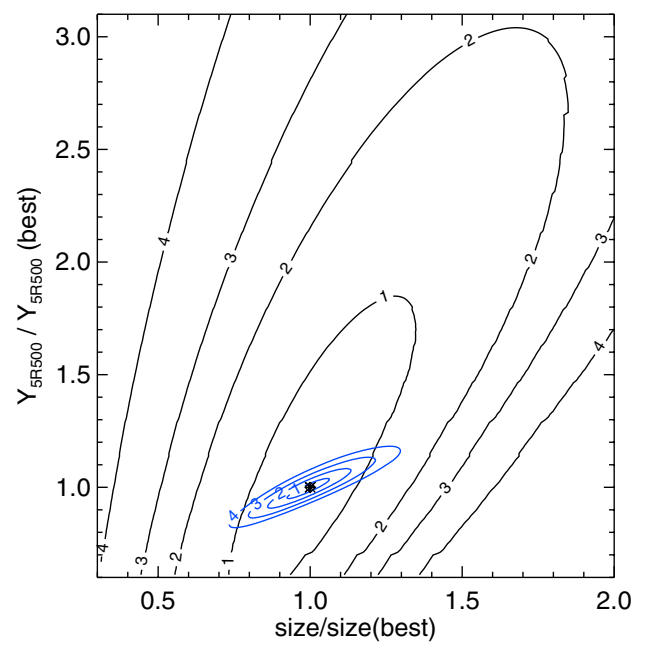

Fig. 9. Illustration of the cluster size- $Y$ degeneracy from PwS. Shown are the cases of Coma cluster (high $S / N$ and extended in blue), and that of an $S / N=6$ unresolved cluster (in black). Parameters are plotted with respect to the best fit points in each direction.

parameter $Y$ is highly correlated with the estimated cluster size. We find a correlation coefficient $\rho=0.91$ and $\rho=0.75$ for Coma and the "unresolved cluster" respectively. On average over the ESZ sample we find a correlation of $\rho=0.85$. The degeneracy between cluster size and $Y$ is extremely detrimental, as it will more than double the average fractional uncertainty relative to the $Y$ value in the case where we knew the true value of $\theta_{\mathrm{s}}$ perfectly. As a result, any attempt to constrain the cluster size (equivalently $\theta_{\mathrm{s}}$ ), fixing or assuming a prior for its value, brings a significant reduction on the $Y$ value dispersion.

The issue of the cluster size- $Y$ degeneracy is of particular importance in the case of Planck, for which a vast majority of clusters are only marginally resolved. This issue is also crucial when one wants to use the SZ signal as a mass proxy. Indeed, the dispersion in $Y$ due to the cluster size- $Y$ degeneracy is likely to dominate the intrinsic scatter of order $10 \%$ of this mass proxy (da Silva et al. 2004; Arnaud et al. 2007).
As a result, we have re-estimated the integrated Compton parameter for all the ESZ candidates with prior information on their sizes. We have chosen the X-ray sizes $\left(\theta_{5 R 500}\right)$ derived from the X-ray luminosities, $L_{500}$, as detailed in Piffaretti et al. (2011), as suitable estimates of the cluster sizes. Using the MMF3 version implemented in HFI Core team and SZ validation team, $Y_{5 R 500}$ were thus re-computed from the Planck channel maps at fixed X-ray position and with imposed cluster size equal to the X-ray luminosity based $\theta_{5 R 500}$. The integrated Compton parameter $Y$ was re-estimated for all the clusters with known $\mathrm{X}$-ray counterparts, being the $158 \mathrm{ESZ}$ candidates identified with known clusters from the MCXC and the nine ESZ clusters confirmed by XMM-Newton as single objects.

Figure 10, left panel, illustrates the effect of fixing the position and the cluster size, in the GNFW profile, to $\theta_{5 R 500}$ for the 158 ESZ identified clusters. The figure displays the measured $Y_{5 R 500}$ values versus the predicted $Y_{5 R 500}^{L_{X}}$ values using X-ray luminosities. The squares stand for integrated Compton parameters obtained from the blind detection whereas the diamonds are integrated Compton parameters re-extracted from the Planck channel maps for the MCXC-identified clusters. Figure 10, right panel, displays the ratio of blind to predicted $Y_{5 R 500}$ versus the ratio of estimated cluster size from blind detection to X-ray cluster size derived from X-ray luminosity. This clearly confirms for the 158 identified clusters that an overestimate of cluster size induces an overestimate of the SZ signal. As seen in the Fig. 10 (left panel), the scatter is significantly reduced from about $43 \%$ to $34 \%$ by imposing a cluster size. Likewise the offset changes from $80 \%$ to $14 \%$.

The dispersion in the predicted integrated Compton parameter is affected by the intrinsic dispersion in the $L_{500}-M$ relation used to derive the predicted SZ quantities as shown in Fig. 11. The selection criterion $S / N \geq 6$ (blue diamonds in the figure) used to construct the ESZ sample indicates that the high $S / N$ clusters are biased towards larger SZ signals, showing that the obtained positive offset in Fig. 10 (left panel) is indeed expected.

As emphasised, a prior on the cluster size helps to break the degeneracy between $Y$ and cluster-size estimates. As a consequence, the better the cluster size estimate, the more reliable the Compton $Y$ parameter estimate. From a selected subsample of 62 ESZ clusters with XMM-Newton archival data (Planck Collaboration 2011g) we have derived accurate estimates of the $\mathrm{X}$-ray sizes, without using the X-ray luminosities, and the $Y_{500}$ were re-evaluated on the Planck channel maps, allowing us to tightly constrain the local SZ versus X-ray scaling properties. As shown in Appendix A of Planck Collaboration (2011g), the scatter is reduced even more than in Fig. 10 (left panel) and no offset is observed any more between the predicted and measured $Y_{500}$ values.

\subsection{Systematic effects}

Due to the cluster size- $Y$ degeneracy discussed above, beam uncertainties are likely to have a significant impact on $Y$ estimates for our candidates because they affect both the original detection and the estimation of cluster size. The beams can be characterised by their shapes and the associated accuracies. The beams for each frequency channel, used for the detection and $Y$ estimate with all methods presented in this study, were assumed Gaussian with FWHM given Planck HFI Core Team (2011b). Uncertainties on the recovered beams have been estimated in Planck HFI Core Team (2011b) and found to range between 1 and $7 \%$ (from 100 to $857 \mathrm{GHz}$ ). These uncertainties on the 

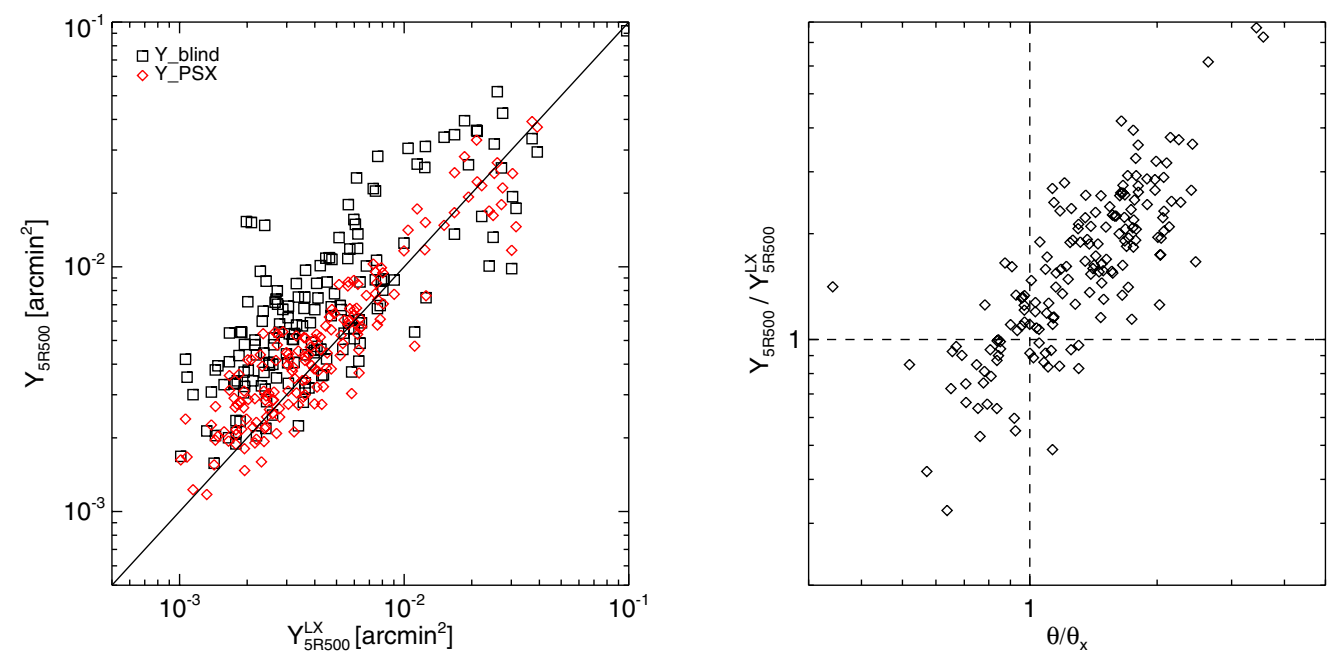

Fig. 10. Left: the scatterplot of the measured integrated Compton parameter $Y_{5 R 500}$ from the $158 \mathrm{X}$-ray identified ESZ clusters against the predicted $Y^{L_{X}}$. Black squares: Estimated cluster size from blind detections. Red diamonds: Re-computed integrated Compton parameter at X-ray positions and with X-ray derived cluster size. Right: the ratio between the $Y$ values and the predicted $Y^{L_{X}}$ against the ratio between the estimated cluster size and the predicted size $\left(\theta / \theta_{X}\right)$.

beams have been propagated to the $Y$ measurements by applying the MMF3 algorithm on the channel maps varying the beam size within the uncertainties at $\pm 1 \sigma$. In doing so we treat differently the ESZ clusters with known X-ray cluster size, for which X-ray positions and estimated $\theta_{5 R 500}$ are fixed, and the ESZ clusters or candidate clusters without estimated cluster size for which the $Y$ were re-estimated without prior information. We find that the uncertainty on the obtained $Y_{5 R 500}$ is of the order of $10 \%$ across the ESZ sample.

The Planck HFI maps used for the cluster extraction are calibrated to better than $2 \%$ for frequencies from 100 to $353 \mathrm{GHz}$, and to better than $7 \%$ beyond (see Planck HFI Core Team 2011 b). This uncertainty in the calibration is accounted for again by performing the SZ-candidate detection with the MMF3 algorithm on the channel maps. We find that on average, the calibration uncertainty propagates into an uncertainty on the $Y$ less than 2\%. The highest Planck-HFI frequencies, with the largest calibration uncertainties, have a low impact on the SZ $Y$ measurement and thus do not impact significantly the overall error budget.

Finally, we have checked that the colour corrections, i.e., the average SZ signal in the HFI bandpasses, induces less than a $3 \%$ difference on the estimated $Y_{5 R 500}$. The SZ-candidate detection and the $Y$ estimates by the MMF3 algorithm were thus performed without taking into account the integration of the SZ spectrum in the Planck bandpasses is negligible.

Table 3 summarises the effects of beam, calibration, and colour correction. It shows that the beam effect is the major source systematic uncertainty in the SZ signal estimate. It is worth noting that the systematic uncertainties are not included in the uncertainties quoted in the ESZ table provided at http:// www.rssd.esa. int/Planck (Planck Collaboration 2011v).

\subsection{Contamination by astrophysical sources}

Galactic and extragalactic sources (both radio and infrared galaxies) are known to lie in the interior of galaxy clusters and hence are a possible source of contamination for the SZ clusters and candidates (Rubiño-Martín \& Sunyaev 2003; Aghanim et al. 2005; Lin et al. 2009).
In the course of ESZ validation, we have gone through an inspection of thirteen known clusters which show some poor quality flags. All these clusters were annotated and the notes can be found in Planck Collaboration (2011v). Ten of them are likely to be contaminated by dust emission from our Galaxy or by IR point sources in their vicinity. Two of them were found to be contaminated by NVSS (at $1.4 \mathrm{GHz}$, Condon et al. 1998) radio sources that are clearly seen in the LFI channels. Combining data from SUMSS (at $0.85 \mathrm{GHz}$, Bock et al. 1999), NVSS, and Planck's LFI and HFI frequencies we find that most radio sources in the ESZ sample have a steep spectrum which makes their contamination to the SZ signal negligible. Three additional clusters (beyond the thirteen), have relatively bright $\left(S_{1.4 \mathrm{GHz}}>0.2 \mathrm{Jy}\right)$ radio sources in their vicinity $(r<15 \mathrm{ar}-$ cmin). NVSS+LFI data reveal flat spectra (indexes between $\alpha=0$ and $\alpha=-0.5$ ). The flux of the radio sources is thus still significant and hence the SZ signal could be affected by their presence.

A statistical analysis has been performed in order to explore the astrophysical contamination over the entire ESZ sample, rather than on an individual cluster basis.

In order to exhibit the initial average level of contamination prior to the use of the MMF algorithm, we have stacked cutouts 4.5 degrees on a side from the channel maps centred at the ESZ cluster/candidate positions from 100 to $857 \mathrm{GHz}$ using a stacking library ${ }^{17}$ detailed in Dole et al. (2006) and Bethermin et al. (2010). The $Y$ values per frequency, obtained from aperture photometry on the stacked cutouts, are displayed in red triangles Fig. 12. The spectral signature normalised to the averaged integrated Compton- $y$ over the whole ESZ sample shows quite good agreement with the theoretical SZ spectrum at low frequencies (Fig. 12, black solid line). Above $353 \mathrm{GHz}$ the signal is highly contaminated by IR emission from Galactic dust and IR point sources.

The $Y$ measurements, per frequency, of the MMF3 algorithm normalised to the integrated Compton- $y$ are averaged over the ESZ sample and the resulting spectral energy distribution is

\footnotetext{
17 http://www.ias.u-psud.fr/irgalaxies/ (Bethermin et al. 2010)
} 


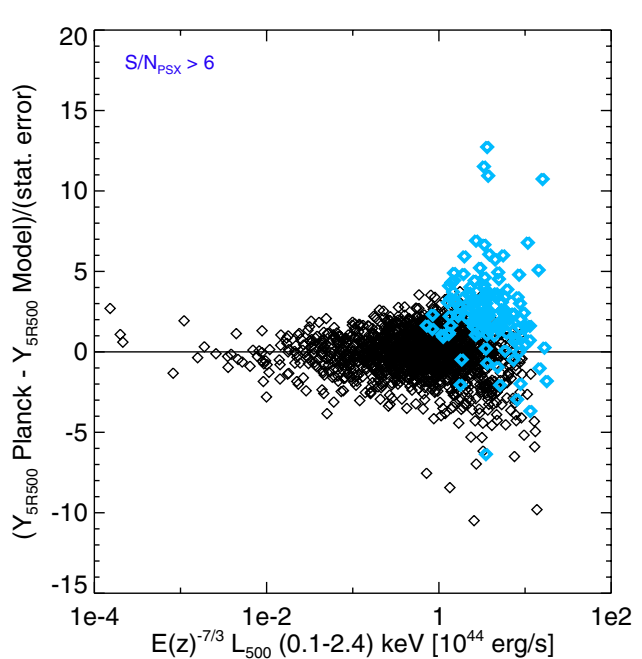

Fig. 11. Ratio of predicted vs. observed $Y_{5 R 500}$ for the MCXC clusters as a function of the X-ray luminosity $L_{500}$ used to estimate the cluster properties (radius and integrated Compton parameter). The light-blue diamonds indicate a cut of 6 in predicted $S / N$ corresponding to the ESZ selection criterion.

Table 3. Systematic error budget on the $Y_{5 R 500}$ values for the ESZ clusters.

\begin{tabular}{lcccc}
\hline \hline Source & Beam & Calibration & $\begin{array}{c}\text { Colour } \\
\text { correction }\end{array}$ & $\begin{array}{c}\text { Astrophysical } \\
\text { contamination }\end{array}$ \\
\hline $\begin{array}{l}\text { Error } \\
\text { contribution }\end{array}$ & $8 \%$ & $2 \%$ & $3 \%$ & $3 \%$ \\
\hline
\end{tabular}

compared with the normalised SZ spectrum (see Fig. 12, blue crosses). The excess of emission at high frequencies is significantly reduced by the filtering technique of the MMF algorithm, reinforcing the idea that most of the excess at the highest frequencies is due to large-scale (larger than the beam) fluctuations in Galaxy emission. The remaining excess after the filtering could be due to a combination of small-scale Galactic fluctuations and/or infrared galaxies. In order to quantify the effect of this residual IR emission on the integrated Compton- $y$ determination, an SZ spectrum was fitted to the averaged spectrum. The normalisation was left free. The displayed error bars contain the dispersion of the measured $Y$ per frequency and, added in quadrature, the uncertainties due to the beam, the colour correction, and the calibration $(\sim 10 \%, \sim 3 \%, \sim 2 \%$ respectively). The best value for the normalised integrated Compton parameter is $Y_{\text {fit }}=1.01$, showing an excellent agreement with the expected spectrum despite the IR excess emission at high frequencies. The same procedure was applied to the $100,143,217$, and $353 \mathrm{GHz}$ $Y$ values and led to $Y_{\mathrm{fit}_{100-353}}=0.97$. This shows that, on average, the residual IR contamination has a negligible effect $(\sim 3 \%)$ on the integrated Compton- $y$ value estimated for the ESZ sample.

\section{Purity and completeness}

The ESZ sample is characterised by the fact that a significant fraction of the clusters and candidate clusters lies near a selection cut. In a catalogue of this sort, the properties of the catalogued clusters will not be representative of the true underlying cluster population. For example, if the SZ signal of a cluster is related to a different cluster property such as mass (collectively referred to as "scaling relations") the observed integrated Compton- $y$

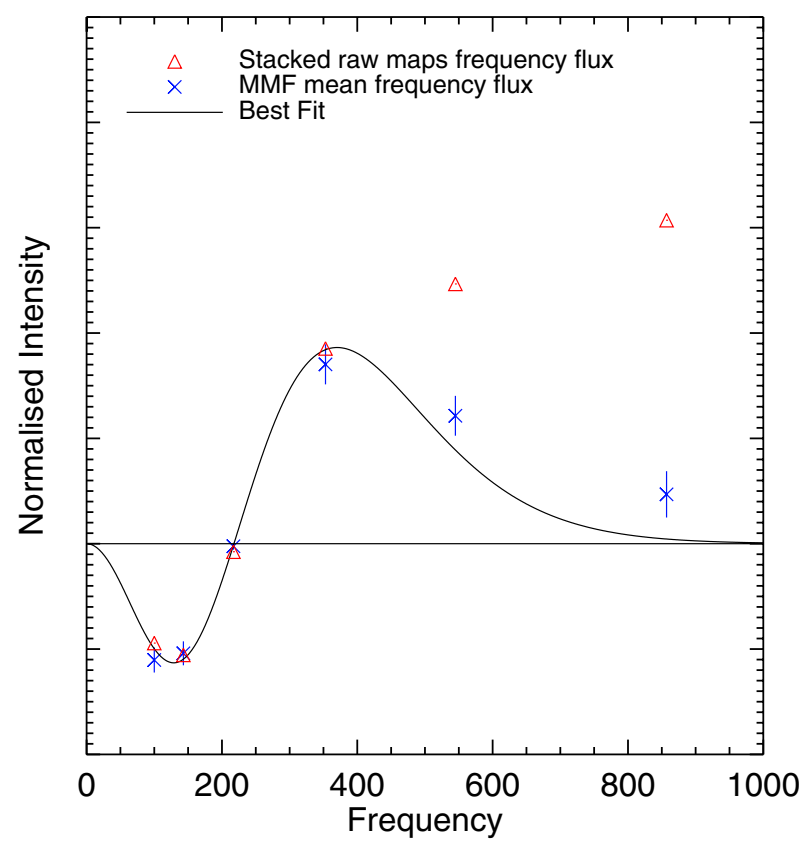

Fig. 12. Average contamination of the ESZ sample by astrophysical sources. Blue crosses: Average $Y$ measurements from MMF3 algorithm normalised to the integrated Compton- $y$. Red triangles: $Y$, obtained from aperture photometry on the stacked cutouts in the channel maps prior filtering by the MMF. Black solid line: Normalised theoretical SZ spectrum.

parameter values, $Y$, will be biased near the selection cut, an effect known as Eddington and Malmquist biases (for discussions in a cluster context, see Mantz et al. 2010 and Andersson et al. 2011).

For the full ESZ sample, we do not always have other cluster properties to relate the integrated Compton- $y$ to, but we can nevertheless examine some statistical effects of selection. In order to do this, we generate large mock cluster catalogues whose properties are designed to mimic those of the observed sample. To impose a selection cut on the mock catalogues, we use the observed relation between $Y_{500}$ and $S / N$ from the region significantly above the selection cut and extrapolate below it, along with an estimate of scatter again from observations. This is carried out in several redshift bins, and leads to a predicted $S / N-Y$ scaling given by

$S / N=10^{1.38 \pm 0.03}(1+z)^{-5.92 \pm 0.24}\left[\frac{Y E^{-2 / 3} D_{A}^{2}}{10^{-4} \mathrm{Mpc}^{2}}\right]$,

with scatter $\sigma_{\log -\log }=0.16$ in log-log scale. We then construct large mock catalogues of clusters through drawing of Poisson samples from the Jenkins et al. (2001) mass function normalised with $\sigma_{8}=0.8$, a value consistent with the latest WMAP constraints. To each cluster and consistent with Planck observations, we assign values of $Y_{5 R 500}$ by adopting the $Y-M$ scaling relation from Planck Collaboration (2011g). An $\mathrm{S} / \mathrm{N}$ value is then assigned as described above, and the cut imposed to create the mock catalogue.

We first use these simulations to estimate the completeness of the ESZ sample as a function of $Y_{5 R 500}$. For clusters within a given bin in $Y_{5 R 500}$, we extract the fraction of mock clusters which lie above the selection cut. The result is shown in Fig. 13 (solid line), and indicates that the sample becomes significantly incomplete (less than $90 \%$ complete) below $Y_{5 R 500} \simeq$ $0.013 \mathrm{arcmin}^{2}$. This result is fairly insensitive to the assumed 
Planck Collaboration: Planck early results. VIII.

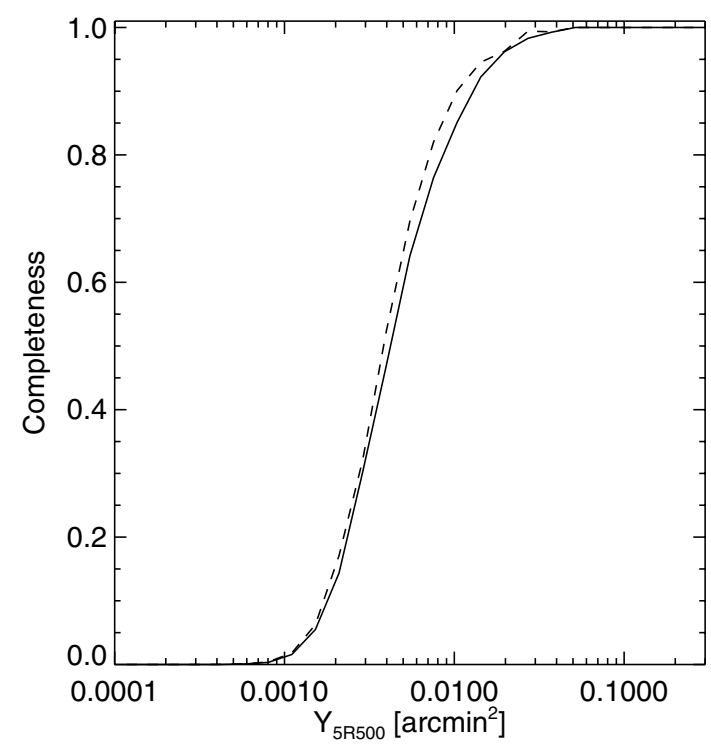

Fig. 13. Expected completeness of the ESZ sample as a function of $Y_{5 R 500}$, estimated from mock cluster catalogues.

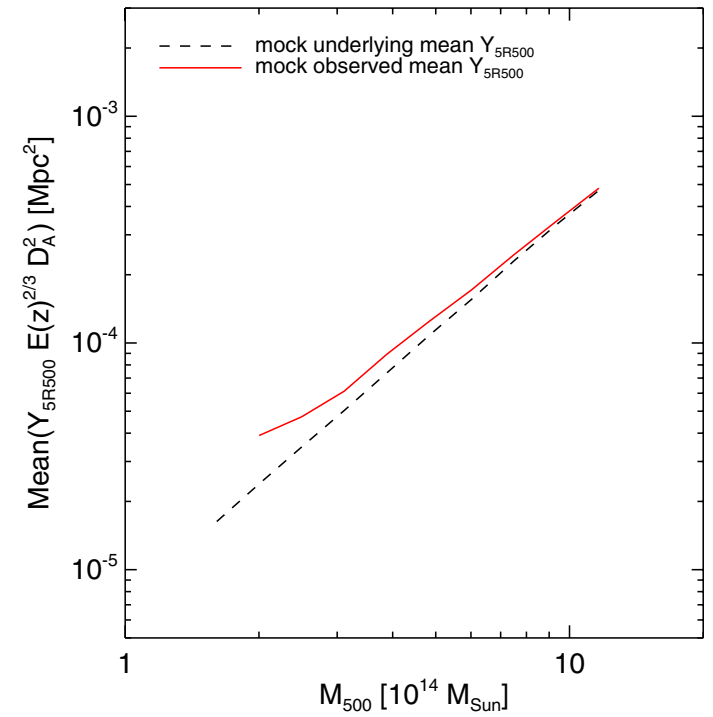

Fig. 14. Expected mean $Y_{5 R 500}$, as a function of cluster mass, for the observed sample and for the predicted underlying cluster sample. At low masses, the observed mean rises above the true mean due to Malmquist bias.

mass function normalisation. For example, changing to $\sigma_{8}=0.9$ (dashed line) causes only small variations in the completeness function. For this case, a completeness of $90 \%$ is obtained at $Y_{5 R 500} \simeq 0.010 \mathrm{arcmin}^{2}$.

We then analyse the extent to which the mean $Y_{5 R 500}$ of the observed clusters is biased in relation to the mean $Y_{5 R 500}$ of the underlying cluster distribution, through those clusters with low $Y_{5 R 500}$ for a given mass being lost via selection. The underlying mean $Y_{5 R 500}$ in the mock samples is given by the input $Y_{500^{-}}$ $M_{500}$ scaling relation from Planck Collaboration (2011g) and the observed $Y_{5 R 500}-Y_{500}$ scaling; as shown in Fig. 14 the mean of the observed clusters will be biased upwards from this, the effect becoming significant for $M_{500}<6 \times 10^{14} M_{\odot}$. Note that this bias does not imply that the $Y_{5 R 500}$ measurements for the ESZ clusters are systematically wrong; the bias is because the selection cut prevents those clusters being representative of the true cluster population at those masses.

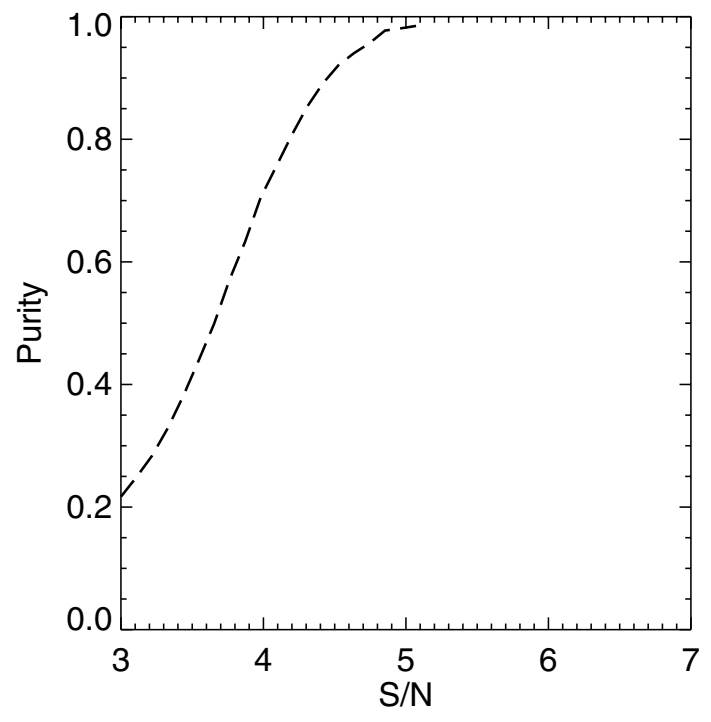

Fig. 15. Purity as a function of $\mathrm{S} / \mathrm{N}$ from $\mathrm{MMF} 3$, based on simulations for the SZ challenge.

Finally, numerical simulations based on the Planck Sky Model were used to estimate the purity of the Planck SZ catalogue. They showed on a simulated sky that a cut in $S / N$ of five ensures $100 \%$ purity of the obtained sample (see Fig. 15). However, the simulation does not capture the entire complexity of the real sky and, in particular, the contamination by astrophysical sources emitting above $217 \mathrm{GHz}$ from IR sources and dust emission or cold cores was found to be higher than expected. The final ESZ sample obtained after applying the selection criterion cut in S/N of 6 contained $190 \mathrm{SZ}$ candidates. The validation of the sample showed that one of them was found to be a spurious source identified with dust emission and it was rejected. The remaining candidate new clusters are to be confirmed. The purity of the ESZ sample thus lies between about $95 \%$ and $99 \%$.

Although an attempt to characterise the completeness and purity is made, we do not provide a fully characterised selection function along with the ESZ sample. The cluster size- $Y$ degeneracy discussed above, together with the large scatter in the contamination level of the SZ detections due mostly to dust emission, makes it difficult to draw a simple relation between the $S / N$ limit used to construct the sample and the measured $Y_{5 R 500}$. It is thus not presently possible to provide a reliable mass limit to our sample. When the telescope beam is larger than the cluster size, a survey is limited by SZ signal. Then, since for the SZ signal the redshift dependence enters through the angular-diameter distance rather than the luminosity distance, the mass selection function is more uniform in SZ than in X-ray surveys. However in our case most of the clusters detected by Planck are at nearby redshifts $\left(z_{\text {median }}=0.15\right)$ and the majority are resolved, adding even more complexity to the selection function.

\section{Statistical characterisation of the ESZ sample}

The ESZ sample is the first all-sky sample of high $S / N$ SZdetected clusters of galaxies produced by Planck. Its high reliability is ensured by the high $S / N$ of the reported detections and by the subsequent validation process. The $S / N$ of the objects in the sample, obtained from blind detection using MMF3 on the reference channel maps, are displayed in Fig. 16. They range between 6 and 29 with median $S / N$ of about eight. Six clusters, including A2163 with $S / N=26$ and Coma with $S / N=22$, 


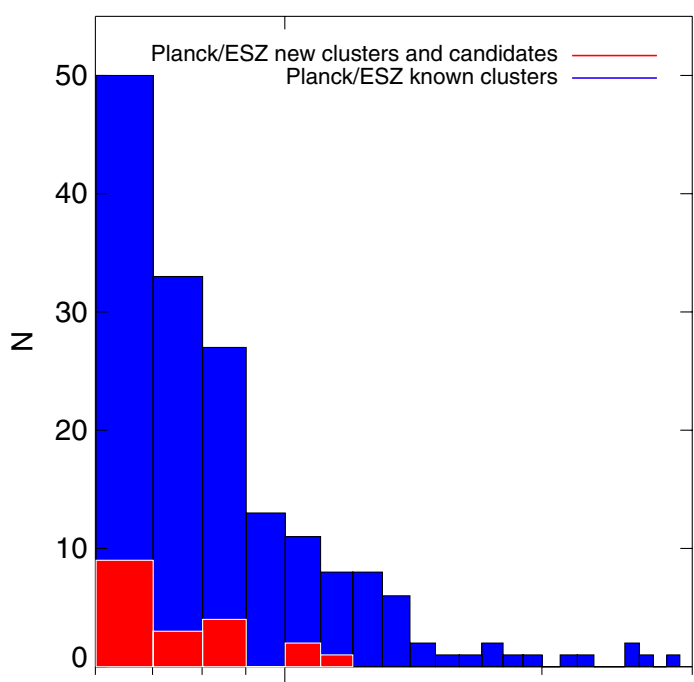

10

$\mathrm{S} / \mathrm{N}$

Fig. 16. Distribution of $S / N$ (for the full ESZ sample: clusters and candidate clusters).

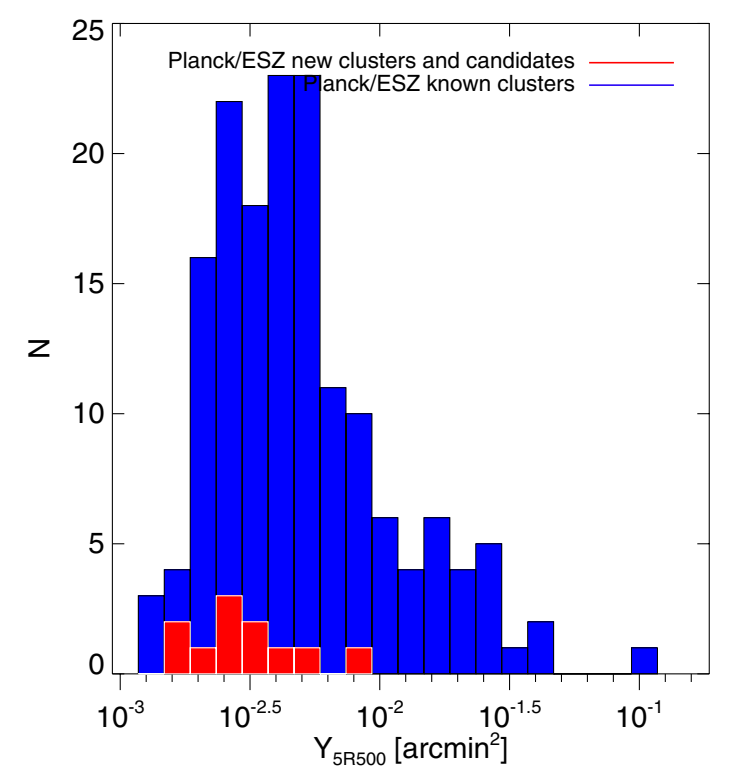

Fig. 17. Distribution of ESZ sample in integrated Compton parameter $Y$.

are in the tail of the $S / N$ distribution with $S / N$ above 20. The twelve confirmed Planck new SZ clusters, included in the ESZ, have their $S / N$ distributed between 6.3 and 11.5. Additional confirmed new clusters with lower $S / N$ are given in Planck Collaboration (2011e). The eight candidate new clusters yet to be confirmed have $S / N$ ranging from 6 to 8.5.

The ESZ provides us with measures of the integrated Compton parameter within a $5 R 500$ sphere, $Y_{5 R 500}$, for 189 clusters or candidates. For about $80 \%$ of the known clusters in the ESZ, this is the very first SZ measure performed in their direction. The integrated Compton parameter of the whole sample, displayed in Fig. 17, shows that the SZ signal extends over about two orders of magnitude from about $1.5 \times 10^{-3}$ to $120 \times 10^{-3} \operatorname{arcmin}^{2}$. Unsurprisingly, the largest value is that of the Coma cluster. Moreover, the estimated cluster sizes from the MMF3 algorithm for the ESZ clusters and candidates are all above $5 \theta_{500}=8$ arcmin, indicating that the high $S / N$ clusters under study can all be considered as extended sources. We compare the estimated cluster size (from blind detection) with the X-ray cluster size obtained from the X-ray observation of the confirmed SZ clusters, considered as a representative cluster size. We find that the SZ blind size is generally larger than the X-ray cluster size; it can be two times larger. As discussed previously, due to the cluster size- $Y$ degeneracy this affects the integrated Compton parameter measurement.

Using the MCXC compilation and the XMM-Newton observations of the confirmed Planck SZ candidates, we obtain masses, $M_{500}$, estimated from mass proxies (luminosity, $L-M$ relation, or $Y_{\mathrm{X}}$ ) for 167 clusters out of the 189 of the ESZ sample. Furthermore, using the redshift information compiled in the MCXC that we retrieved during the validation process and the redshift estimates from XMM-Newton follow-up observations, we gather the redshifts for 173 clusters of the ESZ sample. The distributions of redshifts and masses are exhibited in Figs. 18 and 19, respectively. The redshifts of the ESZ sample are distributed in the range of small to moderate redshifts from about $z=0.01$ to $z=0.55$, with a median redshift of 0.15 . The vast majority of the ESZ clusters, $86 \%$, are thus nearby ones lying below $z=0.3$. Most of the newly-discovered clusters confirmed by XMM-Newton within the ESZ sample have redshifts of the order of 0.4. Among the new Planck clusters confirmed by $X M M-N e w t o n$, but with $S / N$ lower than 6 , released outside the ESZ we find a cluster with $z=0.54$. As for the mass distribution of the ESZ clusters, it spans over a decade with cluster masses ranging from 0.9 to $15 \times 10^{14} M_{\odot}$ within a surveyed volume of the order of $3.5 \times 10^{10} \mathrm{Mpc}^{3}$. It is worth noting that in surveying the whole sky, Planck has a unique capability to detect rare massive clusters in the exponential tail of the mass function. Indeed, among the 21 newly discovered clusters confirmed by XMM-Newton in total (pilot follow-up programme and high- $S$ / $N$ programme) three have total masses of $10 \times 10^{14} M_{\odot}$ or larger and two of them are high $S / N$ clusters in the ESZ sample.

In order to check the consistency of the cosmological model, we compare the measured $Y_{5 R 500}$ with the X-ray predicted $Y_{5 R 500}^{L_{X}}$ that is derived in a given cosmology. To do so, we use the 158 ESZ clusters with X-ray-based size estimates. We vary the cosmological parameter $H_{0}$, in a range of 30 to $100 \mathrm{~km} \mathrm{~s}^{-1} / \mathrm{Mpc}$ assuming a flat universe $\left(\Omega_{\mathrm{m}}=0.3\right.$ and $\left.\Omega_{\Lambda}=1-\Omega_{\mathrm{m}}\right)$. The integrated Compton parameters of the 158 clusters were reestimated from the Planck data with the size $5 R_{500}$ obtained for each explored set of cosmological parameters. The predicted SZ signals are then compared with the SZ signal measured by Planck providing us with the best value for $H_{0}$. We find that $h$ is barely constrained, with a best estimate of $H_{0}=$ $71_{-20}^{+10} \mathrm{~km} \mathrm{~s}^{-1} \mathrm{Mpc}^{-1}$ ( $1 \sigma$ uncertainty).

\subsection{Comparison with existing catalogues}

After the first blind detection of galaxy clusters through their SZ signature by SPT (Staniszewski et al. 2009) and further discoveries by both SPT (Vanderlinde et al. 2010) and ACT (Menanteau et al. 2010), Planck with its broad frequency coverage provides the first sample of SZ clusters detected blindly over the whole sky. For its first and early release, Planck delivers to the community 189 clusters and candidates with $S / N \geq 6$ in the ESZ sample, and an additional ten clusters at lower $S / N$. In total, the 30 new SZ-discovered clusters or candidates by Planck double the number of new clusters provided by ACT and SPT during the last year based on their $455 \mathrm{deg}^{2}$ and $178 \mathrm{deg}^{2}$ respective surveys. Moreover, Planck provides the first homogeneous SZ measurements for many known X-ray or optical clusters. 


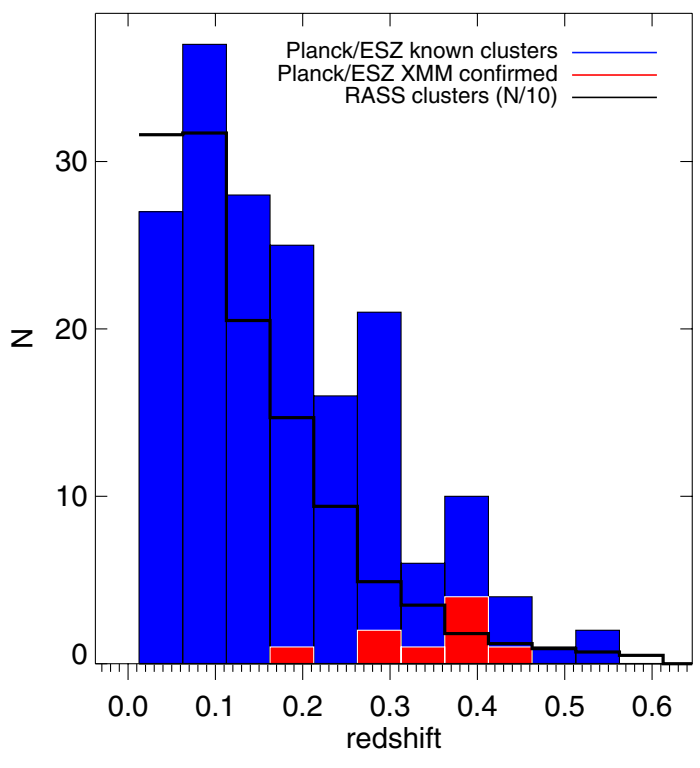

Fig. 18. Distribution of ESZ sample in redshift. The 177 identified ESZ clusters with redshift (from optical or X-ray observations) are in blue, the ESZ clusters confirmed with XMM-Newton in red, and the RASS clusters (number density divided by 10 ) in black solid line.

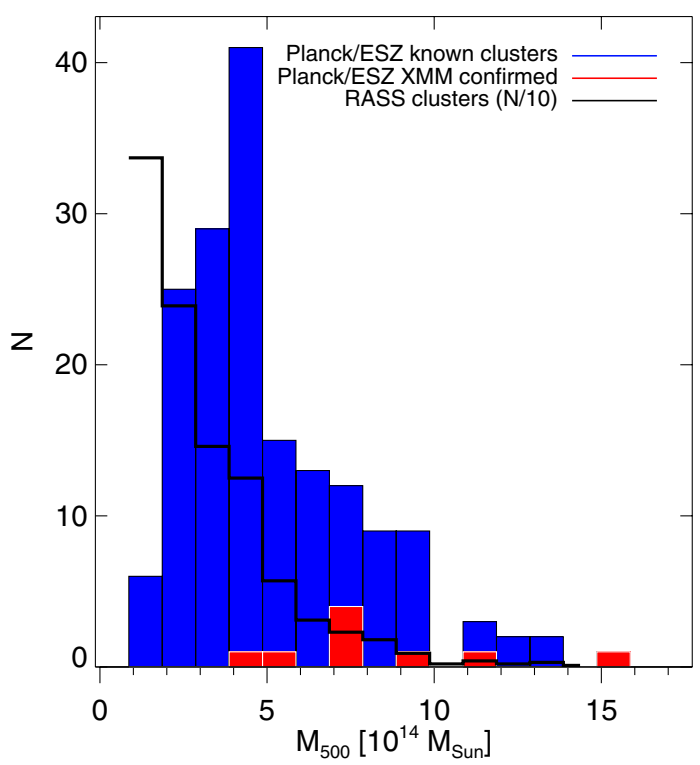

Fig. 19. Distribution of ESZ sample in mass. The 167 identified ESZ clusters with masses are in blue, the ESZ clusters confirmed with $X M M-N e w t o n$ in red, and the RASS clusters (number density divided by 10) in black solid line.

It is worth examining the distribution of the SZ clusters in the $M-z$ plane (see Fig. 20). The range of redshifts covered by the Planck ESZ sample, from $z=0.01$ to 0.55 with more than $80 \%$ of the clusters lying below $z=0.3$, is quite complementary to the high redshift range explored by ACT and SPT experiments, from $z \simeq 0.15$ to 1.2 . The comparison of the estimated masses from the different experiments is complicated by the fact that they are obtained using different approaches, from the use of X-ray proxies to that of mass-significance relations. Overall, we can see from Fig. 20 that the SPT cluster masses quoted in Vanderlinde et al. (2010) range between 1 and $5 \times 10^{14} M_{\odot}$. As mentioned previously, Planck, being an all-sky survey, spans a broader cluster mass range from 0.9 to $10 \times 10^{14} M_{\odot}$ and is particularly adapted to the detection of very massive clusters in the tail of the distribution.

The combination of Planck with ACT and SPT experiments already nicely samples the $M-z$ plane (see Fig. 22). In particular the highest redshift clusters are accessible to ACT and SPT and the most massive clusters to Planck. Moreover, Planck already samples the low-mass low-redshift space quite well and will provide us with a robust reference point in this range. With the deeper observations of the whole sky, combined with appropriate follow-up programmes for redshift estimates, Planck will be able to explore the cluster mass function in its most cosmologically interesting regimes: high redshifts and high masses. However, the detection of the highest redshift clusters is likely to be hampered by the dilution by Planck beam. A combination of the Planck ACT, and SPT carefully taking into account the selection functions of all three experiments will thus be needed to fully take advantage of SZ clusters as a cosmological probe.

Moreover, combining the data from a sample of clusters with different resolutions (including high-resolution imaging of SZ clusters with interferometric experiments like SZA and CARMA) will allow us to perform detailed studies of extended clusters and have a much better handle on the pressure profile from SZ data directly.

Although the ESZ sample is not a catalogue with a fully characterised selection function, it is worth comparing it to the ROSAT-based cluster catalogues. To do this we take advantage of the MCXC, which contains not only NORAS and REFLEX but other survey-based and serendipitous cluster catalogues. Using the homogenised cluster properties of the MCXC compilation, we can moreover predict the SZ signal and the $S / N$ ratio for a measurement of the Compton $Y$ parameter. In order to do this we estimate the Planck noise from real noise maps at the cluster positions using MMF3. Using this information, we compared the number of detected clusters in the ESZ at $S / N \geq 6$ to the number predicted at that level of significance. We find very good overall agreement in terms of detected and predicted clusters, despite the fact that the predictions we use are based on X-ray-selected clusters from the MCXC compilation and that the cluster model used for the prediction does not account for the dispersion in the scaling relations, and despite the noise properties of channel maps being inhomogeneous across the sky. Only 26 MCXC clusters with predicted $S / N \geq 6$ are not within the ESZ sample. For 20 of these clusters information on the presence of a cool core or peculiar morphology is available in the literature. We find that 13 of these host cool cores. For these clusters, the X-ray luminosity is boosted due to the central density peak. The mass predicted from the luminosity, and hence the predicted SZ signal, is over-estimated. For 3 clusters the luminosity measurements adopted in the MCXC are not reliable because of evidence of AGN contamination (e.g., A689). The remaining four clusters are peculiar because they have very asymmetric morphologies or are located in superclusters (e.g., A3526 in Centaurus and the A901/A902 system), making the SZ signal predictions highly uncertain.

There is a large overlap between the Planck ESZ sample and the RASS-based cluster catalogues, in particular REFLEX and NORAS (Fig. 21). The 162 SZ candidates identified with X-ray clusters from the MCXC compilation are predominantly clusters from the REFLEX (74) and NORAS (59) surveys, which corresponds to an overlap of $17 \%$ and $13 \%$ with the REFLEX and NORAS surveys respectively. The eleven ESZ clusters confirmed by $X M M$-Newton with $S / N \geq 6$ were found to lie just around the REFLEX flux limit (only two are above this limit). 


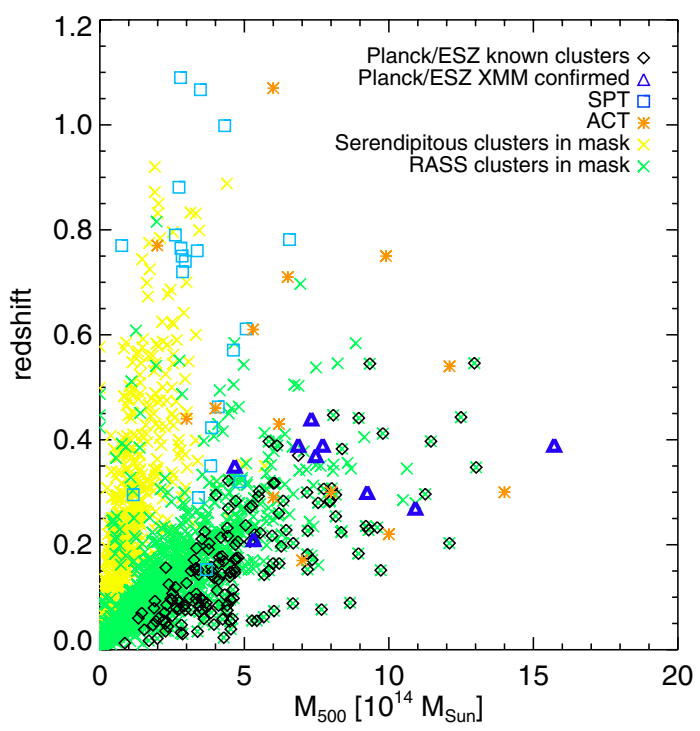

Fig. 20. The 158 clusters from the Planck ESZ sample identified with known X-ray clusters in redshift-mass space, compared with SPT and ACT samples from Menanteau et al. (2010); Vanderlinde et al. (2010), as well as serendipitous and RASS clusters.

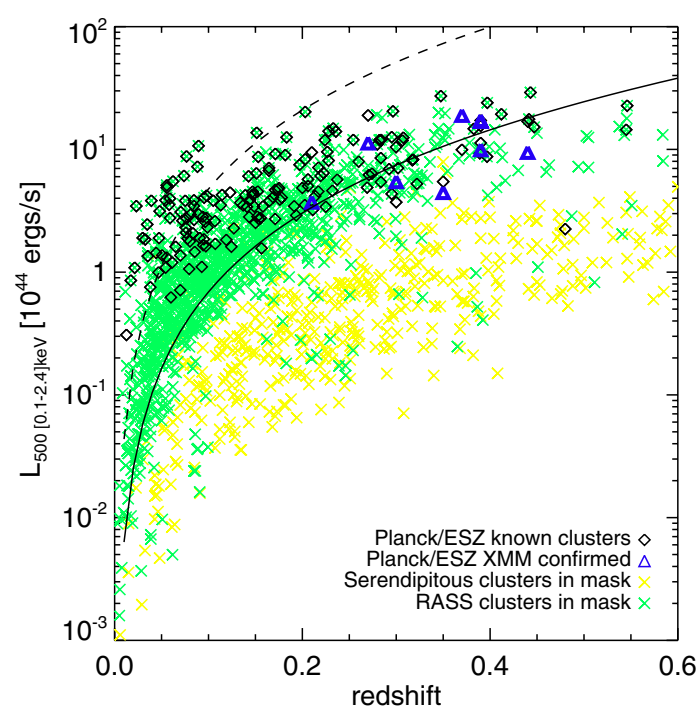

Fig. 21. The 158 clusters from the Planck ESZ sample identified with known X-ray clusters in redshift-luminosity space, compared with serendipitous and RASS clusters.

It is thus interesting to compare the ESZ sample mass and redshift distributions with those of the RASS-based catalogues. This is illustrated in Figs. 18 and 19 in which the RASS-based mass and redshift distribution divided by ten are over-plotted on the ESZ histograms in thick solid line. We find that the ESZ clusters with masses below $4 \times 10^{14} M_{\odot}$ represent only $12 \%$ of the RASS-based clusters in the same mass range; however they represent $90 \%$ of the RASS-based clusters at higher masses $M \geq 9 \times 10^{14} M_{\odot}$. As for the redshift distribution, the Planck ESZ clusters represent $14 \%$ of the RASS-based clusters with redshifts lower than 0.3 and they constitute $31 \%$ of the RASS-based clusters above $z=0.3$.

The SDSS-MaxBCG cluster catalogue is the basis of the study of optical-SZ scaling relations (Planck Collaboration 2011h) in Planck data. It is used in particular to measure an

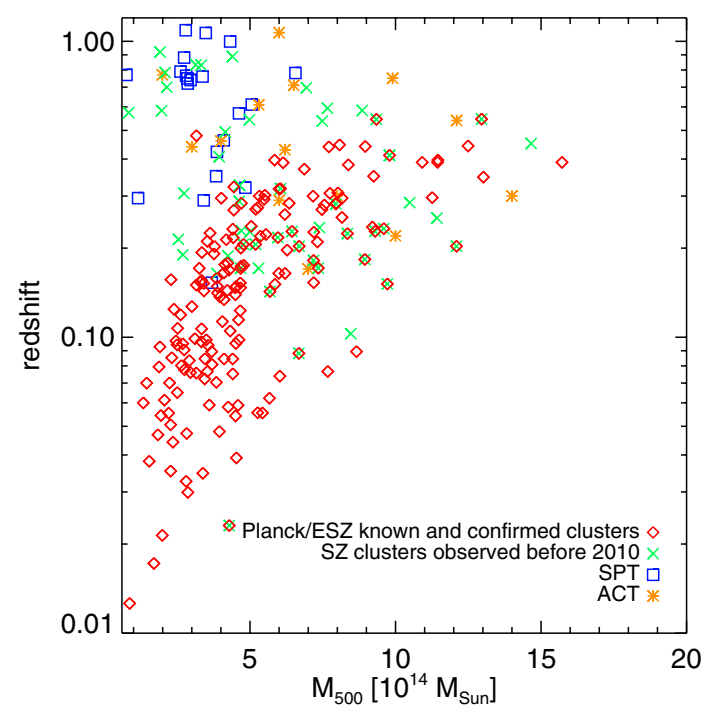

Fig. 22. The ESZ sample compared to the previously observed SZ clusters in redshift-mass space.

integrated Compton parameter, $Y_{5 R 500}^{\mathrm{MaxBCG}}$, from the Planck channel maps at the MaxBCG position using fixed cluster size according to published weak-lensing calibrated mass-richness relations for the MaxBCG catalogue. Only 20 clusters from the MaxBCG have a measured $S / N$ larger than six and are thus expected to be within the ESZ selection ${ }^{18}$. Among them, 18 are effectively associated with ESZ clusters (within a search radius of five arcminutes). One of the two clusters not in the ESZ sample is Abell $1246(z=0.18)$. The second is a fortuitous association with a low-redshift $(z=0.06)$ group of the MaxBCG catalogue near the position of Abell 1795, which is detected in the ESZ catalogue.

\section{Summary}

Thanks to its all-sky coverage and to its frequency range spanning the SZ decrement and increment, Planck provides us with the very first all-sky $S / N$-selected SZ sample. This early release sample of high-reliability SZ clusters and candidates $(S / N$ from 6 to 29) was constructed using a matched multi-filter detection technique. It was validated using Planck-internal quality assessment, external X-ray and optical data, and a multifrequency follow-up programme for confirmation relying mostly on XMM-Newton snapshot observations. The ESZ sample comprises 189 candidates, of which 20 are candidate new clusters and 169 have X-ray or optical counterparts. Of these, 162 were observed in X-ray. Planck provides for the first time SZ observations for about $80 \%$ of the ESZ clusters and hence a homogeneously measured SZ signal. Twelve candidate clusters in total, out of the 20, have been confirmed. One candidate was confirmed by AMI and WISE. Eleven were confirmed with $X M M-N e w t o n$, including two candidates found to be double clusters on the sky.

The clusters in the ESZ sample are mostly at moderate redshifts lying between $z=0.01$ and $z=0.55$, with $86 \%$ of them below $z=0.3$. The ESZ-cluster masses span over a decade from 0.9 to $15 \times 10^{14} M_{\odot}$, i.e. up to the highest masses. The ESZ, constructed using clear selection criteria, is a nearly complete $(90 \%$ above $E^{-2 / 3}(z) Y_{5 R 500} D_{A}^{2} \simeq 4 \times 10^{-4} \mathrm{Mpc}^{2}$ ), high-purity (above

\footnotetext{
18 This number accounts for the possible association of a candidate new cluster with a cluster from Wen et al. (2009).
} 
95\%) SZ cluster sample. However, as mentioned above, it is not possible at the present stage to provide users with a full selection function.

Thanks to its all-sky coverage, Planck has a unique capability to detect the rarest and most massive clusters in the exponential tail of the mass function. Planck is detecting new clusters in a region of the mass-redshift plane that is sparsely populated by the RASS catalogues. As a matter of fact, two of the newlydiscovered clusters in the ESZ and confirmed by XMM-Newton have estimated total masses larger than $10^{15} M_{\odot}$. Furthermore, as indicated by XMM-Newton snapshot observations, most of the new clusters have low luminosity and a disturbed morphology, suggestive of a complex dynamical state. Planck may thus have started to reveal a non-negligible population of massive dynamically-perturbed objects that is under-represented in X-ray surveys.

A significant fraction of the ESZ clusters have good archival X-ray and optical data. In addition, the ESZ sample should motivate follow-up effort by the community. It will hence serve as a valuable reference for studies of cluster physics at low and moderate redshifts (e.g., galaxy properties versus intra-cluster gas physics, metallicities, dynamical state and its evolution, etc). These studies will require multi-wavelength observations including further SZ observations at higher spatial resolution and observations in X-rays (with XMM-Newton, Chandra, and Suzaku), in the optical (imaging and spectroscopy), and in the radio (e.g., with LOFAR).

The ensemble of early results on the SZ signal in Planck using a selected local sub-sample of ESZ clusters with high-quality XMM-Newton archival data (Planck Collaboration 2011g) and using the compilation of about 1600 MCXC clusters (Planck Collaboration 2011f), shows excellent agreement between observed SZ quantities and X-ray-based predictions underlining the robustness and consistency of our overall view of ICM properties. These results shed light on long-standing questions regarding the consistency between the SZ and X-ray view of hot gas in galaxy clusters. In contrast, the SZ signal-to-opticalrichness relation measured from the SDSS-MaxBCG cluster catalogue Planck Collaboration (2011h) has a lower SZ signal than predicted. Extensive SZ-optical statistical studies of this kind are new. The result, and the origin of the difference, may be related to the cluster population, such as the existence of a subpopulation of X-ray under-luminous clusters, or to selection effects in optical cluster catalogues.

In the future, Planck will deliver a larger all-sky SZ cluster catalogue. The characterisation of the Planck selection function together with the construction of this legacy catalogue, including its validation using follow up observations in particular with $X M M-N e w t o n$, will be one of the major activities.

The usefulness of the SZ cluster abundance in achieving precise cosmological constraints relies on several theoretical and observational requirements. One of them is the ability to obtain redshift measurements for each confirmed SZ cluster. Crosscorrelation of Planck data with the only available large optical survey to date, the Sloan Digital Sky Survey (SDSS), can be used to confirm Planck candidates and provide redshift estimates on a area restricted to the SDSS coverage area. The XMM-Newton confirmation observations can provide redshift estimates, but only for the X-ray brightest clusters. A significant follow-up effort in the optical (with ESO, ENO, and NOAO facilities) has thus been put in place by the Planck collaboration in order to obtain redshifts (photometric and spectroscopic) for the SZ clusters. Another key requirement for the cosmological use of the SZ catalogue is the derivation of the fundamental relation between the integrated Compton parameter, $Y$, and the cluster mass and its evolution with redshift. Planck Collaboration (2011g) have calibrated the local relation between $Y$ and $Y_{X}$, the analogue of the SZ signal, measured from the $X$-ray gas mass and temperature, to an unprecedented precision and, for the first time, have demonstrated its remarkably small intrinsic scatter. We will build an even more robust and controlled observational proxy of the cluster mass which is fundamental for cosmological applications. To do this, specific studies based on the comparison of mass estimates from lensing, X-rays and SZ observations for a selected representative sample of the $\mathrm{SZ}$ catalogue will be most crucial.

Finally, combining Planck all-sky SZ data with near future and planned observations of the large-scale structure by large surveys, e.g., PANSTARRS, LOFAR, Euclid, LSST, and e-ROSITA, will allow us to understand the physical processes governing large-scale structure formation and evolution.

Acknowledgements. The authors thank N. Schartel, ESA XMM-Newton project scientist, for granting the Director Discretionary Time used for confirmation of SZ Planck candidates. This research has made use of the following databases: SIMBAD, operated at CDS, Strasbourg, France; the NED database, which is operated by the Jet Propulsion Laboratory, California Institute of Technology, under contract with the National Aeronautics and Space Administration; BAX, operated by the Laboratoire d'Astrophysique de Tarbes-Toulouse (LATT), under contract with the Centre National d'Études Spatiales (CNES), SZ repository operated by IAS Data and Operation Center (IDOC) under contract with CNES. The authors acknowledge the use of software provided by the US National Virtual Observatory. A description of the Planck Collaboration and a list of its members, indicating which technical or scientific activities they have been involved in, can be found at http://www.rssd.esa.int/Planck.

\section{References}

Abell, G. O. 1958, ApJS, 3, 211

Aghanim, N., de Luca, A., Bouchet, F. R., Gispert, R., \& Puget, J. L. 1997, A\&A, 325,9

Aghanim, N., Hansen, S. H., \& Lagache, G. 2005, A\&A, 439, 901

Aghanim, N., Majumdar, S., \& Silk, J. 2008, Rep. Prog. Phys., 71, 066902

Ameglio, S., Borgani, S., Diaferio, A., \& Dolag, K. 2006, MNRAS, 369, 1459

Andersson, K., Benson, B. A., Ade, P. A. R., et al. 2011, ApJ, 738, 48

Appenzeller, I., Thiering, I., Zickgraf, F., et al. 1998, ApJS, 117, 319

Arnaud, M., Pointecouteau, E., \& Pratt, G. W. 2007, A\&A, 474, L37

Arnaud, M., Pratt, G. W., Piffaretti, R., et al. 2010, A\&A, 517, A92

Battistelli, E. S., De Petris, M., Lamagna, L., et al. 2002, ApJ, 580, L101

Battistelli, E. S., De Petris, M., Lamagna, L., et al. 2006, ApJ, 645, 826

Benson, B. A., Church, S. E., Ade, P. A. R., et al. 2003, ApJ, 592, 674

Bethermin, M., Dole, H., Beelen, A., \& Aussel, H. 2010, VizieR Online Data Catalog, 351, 29078

Birkinshaw, M. 1999, Phys. Rep., 310, 97

Birkinshaw, M., \& Gull, S. F. 1978, Nature, 274, 111

Birkinshaw, M., \& Hughes, J. P. 1994, ApJ, 420, 33

Birkinshaw, M., \& Lancaster, K. 2005, in Background Microwave Radiation and Intracluster Cosmology, ed. F. Melchiorri, \& Y. Rephaeli, 127

Bock, D., Large, M. I., \& Sadler, E. M. 1999, AJ, 117, 1578

Böhringer, H., Schuecker, P., Pratt, G. W., et al. 2007, A\&A, 469, 363

Bonamente, M., Joy, M. K., LaRoque, S. J., et al. 2006, ApJ, 647, 25

Borgani, S. 2006 [arXiv: astro-ph/0605575]

Borgani, S., Murante, G., Springel, V., et al. 2004, MNRAS, 348, 1078

Budavári, T., Dobos, L., Szalay, A. S., et al. 2007, in Astronomical Data Analysis Software and Systems XVI, ed. R. A. Shaw, F. Hill, \& D. J. Bell, ASP Conf. Ser., 376, 559

Carlstrom, J. E., Ade, P. A. R., Aird, K. A., et al. 2011, PASP, 123, 568

Carlstrom, J. E., Holder, G. P., \& Reese, E. D. 2002, ARA\&A, 40, 643

Carvalho, P., Rocha, G., \& Hobson, M. P. 2009, MNRAS, 393, 681

Cavaliere, A., \& Fusco-Femiano, R. 1978, A\&A, 70, 677

Colafrancesco, S., Marchegiani, P., \& Palladino, E. 2003, A\&A, 397, 27

Condon, J. J., Cotton, W. D., Greisen, E. W., et al. 1998, AJ, 115, 1693

Cortese, L., Gavazzi, G., Boselli, A., Iglesias-Paramo, J., \& Carrasco, L. 2004, A\&A, 425, 429

da Silva, A. C., Barbosa, D., Liddle, A. R., \& Thomas, P. A. 2001, MNRAS, 326,155 
da Silva, A. C., Kay, S. T., Liddle, A. R., \& Thomas, P. A. 2004, MNRAS, 348, 1401

Dawson, K. S., Holzapfel, W. L., Carlstrom, J. E., et al. 2001, ApJ, 553, L1

De Petris, M., Mainella, G., Nerozzi, A., et al. 1999, New A Rev., 43, 297

Dobbs, M., Halverson, N. W., Ade, P. A. R., et al. 2006, New A Rev., 50, 960

Dole, H., Lagache, G., Puget, J., et al. 2006, A\&A, 451, 417

Douspis, M., Aghanim, N., \& Langer, M. 2006, A\&A, 456, 819

Einasto, M., Einasto, J., Tago, E., Müller, V., \& Andernach, H. 2001, AJ, 122, 2222

Fixsen, D. J., Cheng, E. S., Cottingham, D. A., et al. 1994, ApJ, 420, 457

Génova-Santos, R., Rubiño-Martín, J. A., Rebolo, R., et al. 2005, MNRAS, 363, 79

Ghizzardi, S., Rossetti, M., \& Molendi, S. 2010, A\&A, 516, A32

Glenn, J., Bock, J. J., Chattopadhyay, G., et al. 1998, in SPIE Conf. Ser., 3357, ed. T. G. Phillips, 326

Gómez, P. L., Romer, K. A., Peterson, J., et al. 2003, in SPIE Conf. Ser., 301, ed. S. Bowyer, \& C.-Y. Hwang, 495

Górski, K. M., Hivon, E., Banday, A. J., et al. 2005, ApJ, 622, 759

Grego, L., Carlstrom, J. E., Reese, E. D., et al. 2001, ApJ, 552, 2

Haiman, Z., Mohr, J. J., \& Holder, G. P. 2001, ApJ, 553, 545

Herranz, D., Sanz, J. L., Hobson, M. P., et al. 2002, MNRAS, 336, 1057

Hinshaw, G., Weiland, J. L., Hill, R. S., et al. 2009, ApJS, 180, 225

Holzapfel, W. L., Wilbanks, T. M., Ade, P. A. R., et al. 1997, ApJ, 479, 17

Horellou, C., Nord, M., Johansson, D., \& Lévy, A. 2005, A\&A, 441, 435

Hurier, G., Hildebrandt, S. R., \& Macias-Perez, J. F. 2010 [arXiv: 1007. 1149]

Hurley-Walker, T. A. C. N., Brown, M. L., Davies, M. L., et al. 2011, MNRAS, 414, L75

Itoh, N., Kohyama, Y., \& Nozawa, S. 1998, ApJ, 502, 7

Jenkins, A., Frenk, C. S., White, S. D. M., et al. 2001, MNRAS, 321, 372

Jones, M., Saunders, R., Alexander, P., et al. 1993, Nature, 365, 320

Kashlinsky, A., Atrio-Barandela, F., Kocevski, D., \& Ebeling, H. 2008, ApJ, 686, L49

Kobayashi, S., Sasaki, S., \& Suto, Y. 1996, PASJ, 48, L107

Koester, B. P., McKay, T. A., Annis, J., et al. 2007, ApJ, 660, 239

Komatsu, E., Kitayama, T., Suto, Y., et al. 1999, ApJ, 516, L1

Kravtsov, A. V., Vikhlinin, A., \& Nagai, D. 2006, ApJ, 650, 128

Lamarre, J., Puget, J., Ade, P. A. R., et al. 2010, A\&A, 520, A9

Lamarre, J. M., Giard, M., Pointecouteau, E., et al. 1998, ApJ, 507, L5

Lancaster, K., Genova-Santos, R., Falcòn, N., et al. 2005, MNRAS, 359, 16

Levine, E. S., Schulz, A. E., \& White, M. 2002, ApJ, 577, 569

Lin, Y., Partridge, B., Pober, J. C., et al. 2009, ApJ, 694, 992

Luzzi, G., Shimon, M., Lamagna, L., et al. 2009, ApJ, 705, 1122

Majumdar, S., \& Mohr, J. J. 2004, ApJ, 613, 41

Mantz, A., Allen, S. W., Ebeling, H., Rapetti, D., \& Drlica-Wagner, A. 2010, MNRAS, 406, 1773

Marriage, T. A., Acquaviva, V., Ade, P. A. R., et al. 2011, ApJ, 737, 61

Melin, J., Bartlett, J. G., \& Delabrouille, J. 2006, A\&A, 459, 341

Melin, J.-B., Aghanim, N., Bartelmann, M., et al. 2011, A\&A, submitted

Menanteau, F., González, J., Juin, J., et al. 2010, ApJ, 723, 1523

Mennella, A., Butler, R. C., Curto, A., et al. 2011, A\&A, 536, A3

Miville-Deschênes, M., \& Lagache, G. 2005, ApJS, 157, 302

Montier, L. A., Pelkonen, V., Juvela, M., Ristorcelli, I., \& Marshall, D. J. 2010, A\&A, 522, A83

Motl, P. M., Hallman, E. J., Burns, J. O., \& Norman, M. L. 2005, ApJ, 623, L63

Muchovej, S., Mroczkowski, T., Carlstrom, J. E., et al. 2007, ApJ, 663, 708

Nagai, D., Kravtsov, A. V., \& Vikhlinin, A. 2007, ApJ, 668, 1

Pfrommer, C., Enßlin, T. A., Springel, V., Jubelgas, M., \& Dolag, K. 2007, MNRAS, 378, 385

Piffaretti, R., Arnaud, M., Pratt, G. W., Pointecouteau, E., \& Melin, J. 2011, A\&A, 534, A109

Piffaretti, R., \& Valdarnini, R. 2008, A\&A, 491, 71

Plagge, T., Benson, B. A., Ade, P. A. R., et al. 2010, ApJ, 716, 1118

Planck Collaboration 2011a, A\&A, 536, A1

Planck Collaboration 2011b, A\&A, 536, A2

Planck Collaboration 2011c, A\&A, 536, A7

Planck Collaboration 2011d, A\&A, 536, A8

Planck Collaboration 2011e, A\&A, 536, A9

Planck Collaboration 2011f, A\&A, 536, A10

Planck Collaboration 2011g, A\&A, 536, A11

Planck Collaboration 2011h, A\&A, 536, A12

Planck Collaboration 2011i, A\&A, 536, A13

Planck Collaboration 2011j, A\&A, 536, A14

Planck Collaboration 2011k, A\&A, 536, A15

Planck Collaboration 20111, A\&A, 536, A16

Planck Collaboration 2011m, A\&A, 536, A17

Planck Collaboration 2011n, A\&A, 536, A18

Planck Collaboration 2011o, A\&A, 536, A19

Planck Collaboration 2011p, A\&A, 536, A20
Planck Collaboration 2011q, A\&A, 536, A21

Planck Collaboration 2011r, A\&A, 536, A22

Planck Collaboration 2011s, A\&A, 536, A23

Planck Collaboration 2011t, A\&A, 536, A24

Planck Collaboration 2011u, A\&A, 536, A25

Planck Collaboration 2011v, The Explanatory Supplement to the Planck Early Release Compact Source Catalogue (ESA)

Planck Collaboration 2011w, A\&A, 536, A26

Planck HFI Core Team 2011a, A\&A, 536, A4

Planck HFI Core Team 2011b, A\&A, 536, A6

Pointecouteau, E., Giard, M., \& Barret, D. 1998, A\&A, 336, 44

Pointecouteau, E., Giard, M., Benoit, A., et al. 1999, ApJ, 519, L115

Pratt, G. W., Croston, J. H., Arnaud, M., \& Böhringer, H. 2009, A\&A, 498, 361

Reese, E. D., Carlstrom, J. E., Joy, M., et al. 2002, ApJ, 581, 53

Rephaeli, Y. 1995, ARA\&A, 33, 541

Rubiño-Martín, J. A., \& Sunyaev, R. A. 2003, MNRAS, 344, 1155

Sadat, R., Blanchard, A., Kneib, J., et al. 2004, A\&A, 424, 1097

Schneider, D. P., Bahcall, J. N., Gunn, J. E., \& Dressler, A. 1992, AJ, 103, 1047

Shimon, M., \& Rephaeli, Y. 2004, New A, 9, 69

Silk, J., \& White, S. D. M. 1978, ApJ, 226, L103

Staniszewski, Z., Ade, P. A. R., Aird, K. A., et al. 2009, ApJ, 701, 32

Story, K., Aird, K. A., Andersson, K., et al. 2011, ApJ, 735, L36

Sunyaev, R. A., \& Zeldovich, I. B. 1980, ARA\&A, 18, 537

Sunyaev, R. A., \& Zeldovich, Y. B. 1972, Comments on Astrophysics and Space Physics, 4, 173

Udomprasert, P. S., Mason, B. S., Readhead, A. C. S., \& Pearson, T. J. 2004, ApJ, 615, 63

Uzan, J., Aghanim, N., \& Mellier, Y. 2004, Phys. Rev. D, 70, 083533

Vanderlinde, K., Crawford, T. M., de Haan, T., et al. 2010, ApJ, 722, 1180

Vikhlinin, A., Burenin, R. A., Ebeling, H., et al. 2009, ApJ, 692, 1033

Weller, J., Battye, R. A., \& Kneissl, R. 2002, Phys. Rev. Lett., 88, 231301

Wen, Z. L., Han, J. L., \& Liu, F. S. 2009, ApJS, 183, 197

Williamson, R., Benson, B. A., High, F. W., et al. 2011, ApJ, 738, 139

Wright, E. L., Eisenhardt, P. R. M., Mainzer, A. K., et al. 2010, AJ, 140, 1868

Wu, J., Chiueh, T., Huang, C., et al. 2008, Mod.Phys. Lett. A, 23, 1675

Zemcov, M., Borys, C., Halpern, M., Mauskopf, P., \& Scott, D. 2007, MNRAS, 376, 1073

Zemcov, M., Rex, M., Rawle, T. D., et al. 2010, A\&A, 518, L16

Zwart, J. T. L., Barker, R. W., Biddulph, P., \& AMI Consortium 2008, MNRAS, 391,1545

Zwicky, F., Herzog, E., \& Wild, P. 1961, Catalogue of galaxies and of clusters of galaxies, Vol. I, ed. F. Zwicky, E. Herzog, \& P. Wild

Zacchei, A., Maino, D., Baccigalupi, C., et al. 2011, A\&A, 536, A5

1 Aalto University Metsähovi Radio Observatory, Metsähovintie 114, 02540 Kylmälä, Finland

2 Agenzia Spaziale Italiana Science Data Center, c/o ESRIN, via Galileo Galilei, Frascati, Italy

3 Astroparticule et Cosmologie, CNRS (UMR7164), Université Denis Diderot Paris 7, Bâtiment Condorcet, 10 rue A. Domon et Léonie Duquet, Paris, France

4 Astrophysics Group, Cavendish Laboratory, University of Cambridge, J J Thomson Avenue, Cambridge CB3 OHE, UK

5 Atacama Large Millimeter/submillimeter Array, ALMA Santiago Central Offices, Alonso de Cordova 3107, Vitacura, Casilla 763 0355, Santiago, Chile

${ }^{6}$ CITA, University of Toronto, 60 St. George St., Toronto, ON M5S 3H8, Canada

7 CNRS, IRAP, 9 Av. colonel Roche, BP 44346, 31028 Toulouse Cedex 4, France

8 California Institute of Technology, Pasadena, California, USA

9 Centre of Mathematics for Applications, University of Oslo, Blindern, Oslo, Norway

10 Centro de Astrofísica, Universidade do Porto, Rua das Estrelas, 4150-762 Porto, Portugal

11 DAMTP, University of Cambridge, Centre for Mathematical Sciences, Wilberforce Road, Cambridge CB3 OWA, UK

12 DSM/Irfu/SPP, CEA-Saclay, 91191 Gif-sur-Yvette Cedex, France

13 DTU Space, National Space Institute, Juliane Mariesvej 30, Copenhagen, Denmark

14 Departamento de Física, Universidad de Oviedo, Avda. Calvo Sotelo $\mathrm{s} / \mathrm{n}$, Oviedo, Spain

15 Department of Astronomy and Astrophysics, University of Toronto, 50 Saint George Street, Toronto, Ontario, Canada 
16 Department of Physics \& Astronomy, University of British Columbia, 6224 Agricultural Road, Vancouver, British Columbia, Canada

17 Department of Physics and Astronomy, University of Southern California, Los Angeles, California, USA

18 Department of Physics and Astronomy, University of Sussex, Brighton BN1 9QH, UK

19 Department of Physics, Gustaf Hällströmin katu 2a, University of Helsinki, Helsinki, Finland

20 Department of Physics, Princeton University, Princeton, New Jersey, USA

21 Department of Physics, Purdue University, 525 Northwestern Avenue, West Lafayette, Indiana, USA

22 Department of Physics, University of California, Berkeley, California, USA

23 Department of Physics, University of California, One Shields Avenue, Davis, California, USA

24 Department of Physics, University of California, Santa Barbara, California, USA

25 Department of Physics, University of Illinois at Urbana-Champaign, 1110 West Green Street, Urbana, Illinois, USA

26 Dipartimento di Fisica G. Galilei, Università degli Studi di Padova, via Marzolo 8, 35131 Padova, Italy

27 Dipartimento di Fisica, Università La Sapienza, P.le A. Moro 2, Roma, Italy

28 Dipartimento di Fisica, Università degli Studi di Milano, via Celoria 16, Milano, Italy

29 Dipartimento di Fisica, Università degli Studi di Trieste, via A. Valerio 2, Trieste, Italy

30 Dipartimento di Fisica, Università di Ferrara, via Saragat 1, 44122 Ferrara, Italy

31 Dipartimento di Fisica, Università di Roma Tor Vergata, via della Ricerca Scientifica 1, Roma, Italy

32 Discovery Center, Niels Bohr Institute, Blegdamsvej 17, Copenhagen, Denmark

33 Dpto. Astrofísica, Universidad de La Laguna (ULL), 38206 La Laguna, Tenerife, Spain

34 European Southern Observatory, ESO Vitacura, Alonso de Cordova 3107, Vitacura, Casilla 19001, Santiago, Chile

35 European Space Agency, ESAC, Camino bajo del Castillo, s/n, Urbanización Villafranca del Castillo, Villanueva de la Cañada, Madrid, Spain

36 European Space Agency, ESAC, Planck Science Office, Camino bajo del Castillo s/n, Urbanización Villafranca del Castillo, Villanueva de la Cañada, Madrid, Spain

37 European Space Agency, ESTEC, Keplerlaan 1, 2201 AZ Noordwijk, The Netherlands

38 GEPI, Observatoire de Paris, Section de Meudon, 5 place J. Janssen, 92195 Meudon Cedex, France

39 Helsinki Institute of Physics, Gustaf Hällströmin katu 2, University of Helsinki, Helsinki, Finland

40 INAF - Osservatorio Astronomico di Padova, Vicolo dell'Osservatorio 5, Padova, Italy

41 INAF - Osservatorio Astronomico di Roma, via di Frascati 33, Monte Porzio Catone, Italy

42 INAF - Osservatorio Astronomico di Trieste, via G.B. Tiepolo 11, Trieste, Italy

43 INAF/IASF Bologna, via Gobetti 101, Bologna, Italy

44 INAF/IASF Milano, via E. Bassini 15, Milano, Italy

45 INRIA, Laboratoire de Recherche en Informatique, Université Paris-Sud 11, Bâtiment 490, 91405 Orsay Cedex, France

46 IPAG (Institut de Planétologie et d'Astrophysique de Grenoble), Université Joseph Fourier, Grenoble 1 / CNRS-INSU, UMR 5274, Grenoble 38041, France

47 Imperial College London, Astrophysics group, Blackett Laboratory, Prince Consort Road, London, SW7 2AZ, UK

48 Infrared Processing and Analysis Center, California Institute of Technology, Pasadena, CA 91125, USA

49 Institut Néel, CNRS, Université Joseph Fourier Grenoble I, 25 rue des Martyrs, Grenoble, France
50 Institut d'Astrophysique Spatiale, CNRS (UMR8617) Université Paris-Sud 11, Bâtiment 121, Orsay, France

51 Institut d'Astrophysique de Paris, CNRS UMR7095, Université Pierre \& Marie Curie, 98bis boulevard Arago, Paris, France

52 Institut de Ciències de l'Espai, CSIC/IEEC, Facultat de Ciències, Campus UAB, Torre C5 par-2, Bellaterra 08193, Spain

53 Institute of Astronomy and Astrophysics, Academia Sinica, Taipei, Taiwan

54 Institute of Astronomy, University of Cambridge, Madingley Road, Cambridge CB3 0HA, UK

55 Institute of Theoretical Astrophysics, University of Oslo, Blindern, Oslo, Norway

56 Instituto de Astrofísica de Canarias, C/vía Láctea s/n, La Laguna, Tenerife, Spain

${ }^{57}$ Instituto de Física de Cantabria (CSIC-Universidad de Cantabria), Avda. de los Castros s/n, Santander, Spain

58 Jet Propulsion Laboratory, California Institute of Technology, 4800 Oak Grove Drive, Pasadena, California, USA

59 Jodrell Bank Centre for Astrophysics, Alan Turing Building, School of Physics and Astronomy, The University of Manchester, Oxford Road, Manchester, M13 9PL, UK

60 Kavli Institute for Cosmology Cambridge, Madingley Road, Cambridge, CB3 OHA, UK

61 LERMA, CNRS, Observatoire de Paris, 61 Avenue de l'Observatoire, Paris, France

62 Laboratoire AIM, IRFU/Service d'Astrophysique - CEA/DSM CNRS - Université Paris Diderot, Bât. 709, CEA-Saclay, 91191 Gifsur-Yvette Cedex, France

${ }^{63}$ Laboratoire Traitement et Communication de l'Information, CNRS (UMR 5141) and Télécom ParisTech, 46 rue Barrault, 75634 Paris Cedex 13, France

${ }^{64}$ Laboratoire de Physique Subatomique et de Cosmologie, CNRS/IN2P3, Université Joseph Fourier Grenoble I, Institut National Polytechnique de Grenoble, 53 rue des Martyrs, 38026 Grenoble Cedex, France

${ }^{65}$ Laboratoire de l'Accélérateur Linéaire, Université Paris-Sud 11, CNRS/IN2P3, Orsay, France

${ }^{66}$ Lawrence Berkeley National Laboratory, Berkeley, California, USA

${ }^{67}$ MPA Partner Group, Key Laboratory for Research in Galaxies and Cosmology, Shanghai Astronomical Observatory, Chinese Academy of Sciences, Nandan Road 80, Shanghai 200030, PR China

68 Max-Planck-Institut für Astrophysik, Karl-Schwarzschild-Str. 1, 85741 Garching, Germany

${ }^{69}$ Max-Planck-Institut für Extraterrestrische Physik, Giessenbachstraße, 85748 Garching, Germany

70 MilliLab, VTT Technical Research Centre of Finland, Tietotie 3, Espoo, Finland

71 National University of Ireland, Department of Experimental Physics, Maynooth, Co. Kildare, Ireland

72 Niels Bohr Institute, Blegdamsvej 17, Copenhagen, Denmark

73 Observational Cosmology, Mail Stop 367-17, California Institute of Technology, Pasadena, CA, 91125, USA

74 Optical Science Laboratory, University College London, Gower Street, London, UK

75 SISSA, Astrophysics Sector, via Bonomea 265, 34136 Trieste, Italy

76 SUPA, Institute for Astronomy, University of Edinburgh, Royal Observatory, Blackford Hill, Edinburgh EH9 3HJ, UK

77 School of Physics and Astronomy, Cardiff University, Queens Buildings, The Parade, Cardiff CF24 3AA, UK

78 Space Research Institute (IKI), Russian Academy of Sciences, Profsoyuznaya Str, 84/32, Moscow, 117997, Russia

79 Space Sciences Laboratory, University of California, Berkeley, California, USA

80 Stanford University, Dept of Physics, Varian Physics Bldg, 382 via Pueblo Mall, Stanford, California, USA

81 Tartu Observatory, Toravere, Tartumaa, 61602, Estonia

82 Tuorla Observatory, Department of Physics and Astronomy, University of Turku, Väisäläntie 20, 21500, Piikkiö, Finland 
83 Universität Heidelberg, Institut für Theoretische Astrophysik, Albert-Überle-Str. 2, 69120 Heidelberg, Germany

${ }^{84}$ Université Denis Diderot (Paris 7), 75205 Paris Cedex 13, France

85 Université de Toulouse, UPS-OMP, IRAP, 31028 Toulouse Cedex 4 , France

86 Universities Space Research Association, Stratospheric Observatory for Infrared Astronomy, MS 211-3, Moffett Field, CA 94035, USA
87 University Observatory, Ludwig Maximilian University of Munich, Scheinerstrasse 1, 81679 Munich, Germany

88 University of Granada, Departamento de Física Teórica y del Cosmos, Facultad de Ciencias, Granada, Spain

89 University of Miami, Knight Physics Building, 1320 Campo Sano Dr., Coral Gables, Florida, USA

90 Warsaw University Observatory, Aleje Ujazdowskie 4, 00-478 Warszawa, Poland 


\section{Appendix A: ESZ sample extract}

Table A.1 is an extract from the Planck ESZ sample available at www.rssd.esa.int/Planck aiming at presenting the content of the released product. Four entries are given as examples for each category (Planck ESZ known clusters, Planck ESZ new confirmed clusters, Planck ESZ clusters candidates). In the present extract, only Galactic longitudes and latitudes are given. The ESZ sample contains, in addition, the right ascensions and declinations for all the entries.

For each entry the following fields are provided:

- name: Planck Name of Cluster Candidate;

- GLON: Galactic Longitude from Planck;

- GLAT: Galactic latitude from Planck;

- $S / N$ : Signal-to-noise ratio returned by the matched multiFilter algorithm (MMF3);

- ID: external Identifier of Planck Clusters e.g. Coma, Abell 2163 etc.;

- z: redshift of Cluster from the MCXC X-ray cluster compilation unless otherwise stated in the individual notes;

- $\Theta_{X}$ : angular size at $5 R 500$ from X-ray data;

- $Y_{P S X}$ : integrated Compton parameter at X-ray position and within $5 R 500\left(\Theta_{X}\right)$ in $\operatorname{arcmin}^{2}$;

- $Y_{P S X}^{E R R}$ : uncertainty in Integrated Compton parameter at X-ray position and within $5 R 500\left(\Theta_{X}\right)$ in $\operatorname{arcmin}^{2}$;

- $\Theta$ : estimated angular size from matched multi-Filter (MMF3),

- Y: integrated Compton parameter at Planck position and within $\Theta$, from matched multi-Filter (MMF3) in $\operatorname{arcmin}^{2}$;

- $Y^{E R R}$ : uncertainty in Integrated Compton parameter at Planck position and within $\Theta$ from matched multiFilter (MMF3) in $\operatorname{arcmin}^{2}$. 
Table A.1. ESZ sample.

\begin{tabular}{|c|c|c|c|c|c|c|c|c|c|c|c|}
\hline Name & GLON & GLAT & $S / N$ & ID & $\mathrm{Z}$ & $\Theta_{X}$ & $Y_{P S X}$ & $Y_{P S X}^{E R R}$ & $\Theta$ & $Y$ & $Y^{E R R}$ \\
\hline PLCKG111.0+31.7 & 110.98 & 31.73 & 28.93 & A2256 & 0.06 & $\mathrm{NaN}$ & 0.0242 & 0.0009 & $\mathrm{NaN}$ & $\mathrm{NaN}$ & $\mathrm{NaN}$ \\
\hline PLCKG57.3+88.0 & 57.34 & 88.01 & 21.94 & Coma & 0.02 & $\mathrm{NaN}$ & 0.1173 & 0.0054 & $\mathrm{NaN}$ & $\mathrm{NaN}$ & $\mathrm{NaN}$ \\
\hline PLCKG239.3+24.8 & 239.28 & 24.77 & 25.67 & A0754 & 0.05 & $\mathrm{NaN}$ & 0.0330 & 0.0012 & $\mathrm{NaN}$ & $\mathrm{NaN}$ & $\mathrm{NaN}$ \\
\hline PLCKG272.1-40.2 & 272.11 & -40.15 & 25.90 & A3266 & 0.06 & $\mathrm{NaN}$ & 0.0282 & 0.0012 & $\mathrm{NaN}$ & $\mathrm{NaN}$ & $\mathrm{NaN}$ \\
\hline PLCKG6.8+30.5 & 6.78 & 30.47 & 26.40 & A2163 & 0.20 & $\mathrm{NaN}$ & 0.0173 & 0.0007 & $\mathrm{NaN}$ & $\mathrm{NaN}$ & $\mathrm{NaN}$ \\
\hline PLCKG340.9-33.3 & 340.89 & -33.35 & 22.02 & A3667 & 0.06 & $\mathrm{NaN}$ & 0.0266 & 0.0014 & $\mathrm{NaN}$ & $\mathrm{NaN}$ & $\mathrm{NaN}$ \\
\hline PLCKG266.0-21.3 & 266.04 & -21.25 & 19.75 & 1ES 0657-55.8 & 0.30 & $\mathrm{NaN}$ & 0.0067 & 0.0003 & $\mathrm{NaN}$ & $\mathrm{NaN}$ & $\mathrm{NaN}$ \\
\hline PLCKG44.2+48.7 & 44.23 & 48.68 & 18.46 & A2142 & 0.09 & $\mathrm{NaN}$ & 0.0241 & 0.0013 & $\mathrm{NaN}$ & $\mathrm{NaN}$ & $\mathrm{NaN}$ \\
\hline PLCKG93.9+34.9 & 93.92 & 34.91 & 17.31 & A2255 & 0.08 & $\mathrm{NaN}$ & 0.0103 & 0.0006 & $\mathrm{NaN}$ & $\mathrm{NaN}$ & $\mathrm{NaN}$ \\
\hline PLCKG164.2-38.9 & 164.19 & -38.89 & 13.79 & A0401 & 0.07 & $\mathrm{NaN}$ & 0.0193 & 0.0016 & $\mathrm{NaN}$ & $\mathrm{NaN}$ & $\mathrm{NaN}$ \\
\hline PLCKG72.6+41.5 & 72.63 & 41.46 & 17.44 & A2219 & 0.23 & $\mathrm{NaN}$ & 0.0085 & 0.0005 & $\mathrm{NaN}$ & $\mathrm{NaN}$ & $\mathrm{NaN}$ \\
\hline PLCKG263.7-22.5 & 263.67 & -22.54 & 16.70 & A3404 & 0.16 & $\mathrm{NaN}$ & 0.0064 & 0.0004 & $\mathrm{NaN}$ & $\mathrm{NaN}$ & $\mathrm{NaN}$ \\
\hline PLCKG97.7+38.1 & 97.74 & 38.12 & 14.65 & A2218 & 0.17 & $\mathrm{NaN}$ & 0.0044 & 0.0003 & $\mathrm{NaN}$ & $\mathrm{NaN}$ & $\mathrm{NaN}$ \\
\hline PLCKG263.2-25.2 & 263.21 & -25.21 & 11.24 & A3395 & 0.05 & $\mathrm{NaN}$ & 0.0073 & 0.0009 & $\mathrm{NaN}$ & $\mathrm{NaN}$ & $\mathrm{NaN}$ \\
\hline PLCKG262.3-35.4 & 262.25 & -35.37 & 15.19 & ACO S0520 & 0.30 & $\mathrm{NaN}$ & 0.0034 & 0.0003 & $\mathrm{NaN}$ & $\mathrm{NaN}$ & $\mathrm{NaN}$ \\
\hline PLCKG74.0-27.8 & 73.97 & -27.82 & 14.25 & A2390 & 0.23 & $\mathrm{NaN}$ & 0.0056 & 0.0005 & $\mathrm{NaN}$ & $\mathrm{NaN}$ & $\mathrm{NaN}$ \\
\hline PLCKG332.2-46.4 & 332.23 & -46.37 & 13.89 & A3827 & 0.10 & $\mathrm{NaN}$ & 0.0086 & 0.0007 & $\mathrm{NaN}$ & $\mathrm{NaN}$ & $\mathrm{NaN}$ \\
\hline PLCKG265.0-48.9 & 265.01 & -48.95 & 13.95 & A3158 & 0.06 & $\mathrm{NaN}$ & 0.0117 & 0.0010 & $\mathrm{NaN}$ & $\mathrm{NaN}$ & $\mathrm{NaN}$ \\
\hline PLCKG115.2-72.1 & 115.16 & -72.09 & 13.14 & A0085 & 0.06 & $\mathrm{NaN}$ & 0.0210 & 0.0018 & $\mathrm{NaN}$ & $\mathrm{NaN}$ & $\mathrm{NaN}$ \\
\hline PLCKG316.3+28.5 & 316.35 & 28.54 & 12.85 & A3571 & 0.04 & $\mathrm{NaN}$ & 0.0372 & 0.0031 & $\mathrm{NaN}$ & $\mathrm{NaN}$ & $\mathrm{NaN}$ \\
\hline PLCKG86.5+15.3 & 86.46 & 15.30 & 12.33 & CIZA J1938.3+5409 & 0.26 & $\mathrm{NaN}$ & 0.0031 & 0.0003 & $\mathrm{NaN}$ & $\mathrm{NaN}$ & $\mathrm{NaN}$ \\
\hline PLCKG33.8+77.2 & 33.78 & 77.16 & 12.39 & A1795 & 0.06 & $\mathrm{NaN}$ & 0.0169 & 0.0014 & $\mathrm{NaN}$ & $\mathrm{NaN}$ & $\mathrm{NaN}$ \\
\hline PLCKG6.5+50.5 & 6.48 & 50.55 & 13.36 & A2029 & 0.08 & $\mathrm{NaN}$ & 0.0180 & 0.0015 & $\mathrm{NaN}$ & $\mathrm{NaN}$ & $\mathrm{NaN}$ \\
\hline PLCKG349.5-59.9 & 349.46 & -59.95 & 13.93 & ACO S1063 & 0.35 & $\mathrm{NaN}$ & 0.0046 & 0.0003 & $\mathrm{NaN}$ & $\mathrm{NaN}$ & $\mathrm{NaN}$ \\
\hline PLCKG186.4+37.3 & 186.39 & 37.26 & 12.61 & A0697 & 0.28 & $\mathrm{NaN}$ & 0.0051 & 0.0005 & $\mathrm{NaN}$ & $\mathrm{NaN}$ & $\mathrm{NaN}$ \\
\hline PLCKG229.9+15.3 & 229.94 & 15.30 & 12.46 & A0644 & 0.07 & $\mathrm{NaN}$ & 0.0116 & 0.0010 & $\mathrm{NaN}$ & $\mathrm{NaN}$ & $\mathrm{NaN}$ \\
\hline PLCKG149.7+34.7 & 149.73 & 34.70 & 11.57 & A0665 & 0.18 & $\mathrm{NaN}$ & 0.0060 & 0.0005 & $\mathrm{NaN}$ & $\mathrm{NaN}$ & $\mathrm{NaN}$ \\
\hline PLCKG3.9-59.4 & 3.91 & -59.42 & 12.06 & A3888 & 0.15 & $\mathrm{NaN}$ & 0.0061 & 0.0005 & $\mathrm{NaN}$ & $\mathrm{NaN}$ & $\mathrm{NaN}$ \\
\hline PLCKG312.0+30.7 & 312.00 & 30.72 & 9.04 & A 3558 & 0.05 & $\mathrm{NaN}$ & 0.0223 & 0.0024 & $\mathrm{NaN}$ & $\mathrm{NaN}$ & $\mathrm{NaN}$ \\
\hline PLCKG313.9-17.1 & 313.87 & -17.11 & 11.57 & CIZA J1601.7-7544 & 0.15 & $\mathrm{NaN}$ & 0.0078 & 0.0007 & $\mathrm{NaN}$ & $\mathrm{NaN}$ & $\mathrm{NaN}$ \\
\hline PLCKG335.6-46.5 & 335.59 & -46.46 & 10.17 & A3822 & 0.08 & $\mathrm{NaN}$ & 0.0084 & 0.0008 & $\mathrm{NaN}$ & $\mathrm{NaN}$ & $\mathrm{NaN}$ \\
\hline PLCKG288.6-37.7 & 288.62 & -37.66 & 9.86 & A3186 & 0.13 & $\mathrm{NaN}$ & 0.0053 & 0.0006 & $\mathrm{NaN}$ & $\mathrm{NaN}$ & $\mathrm{NaN}$ \\
\hline PLCKG315.7-18.0 & 315.71 & -18.04 & 11.44 & A3628 & 0.10 & $\mathrm{NaN}$ & 0.0088 & 0.0008 & $\mathrm{NaN}$ & $\mathrm{NaN}$ & $\mathrm{NaN}$ \\
\hline PLCKG263.2-23.4 & 263.16 & -23.41 & 10.08 & ACO S0592 & 0.23 & $\mathrm{NaN}$ & 0.0032 & 0.0003 & $\mathrm{NaN}$ & $\mathrm{NaN}$ & $\mathrm{NaN}$ \\
\hline PLCKG149.2+54.2 & 149.24 & 54.19 & 11.58 & A1132 & 0.14 & $\mathrm{NaN}$ & 0.0052 & 0.0005 & $\mathrm{NaN}$ & $\mathrm{NaN}$ & $\mathrm{NaN}$ \\
\hline PLCKG21.1+33.3 & 21.09 & 33.26 & 10.61 & A2204 & 0.15 & $\mathrm{NaN}$ & 0.0076 & 0.0007 & $\mathrm{NaN}$ & $\mathrm{NaN}$ & $\mathrm{NaN}$ \\
\hline PLCKG322.0-48.0 & 321.96 & -47.98 & 11.27 & A3921 & 0.09 & $\mathrm{NaN}$ & 0.0053 & 0.0006 & $\mathrm{NaN}$ & $\mathrm{NaN}$ & $\mathrm{NaN}$ \\
\hline PLCKG182.4-28.3 & 182.44 & -28.30 & 12.77 & A0478 & 0.09 & $\mathrm{NaN}$ & 0.0167 & 0.0014 & $\mathrm{NaN}$ & $\mathrm{NaN}$ & $\mathrm{NaN}$ \\
\hline PLCKG242.0+14.9 & 241.97 & 14.86 & 10.49 & A3411 & 0.17 & $\mathrm{NaN}$ & 0.0041 & 0.0005 & $\mathrm{NaN}$ & $\mathrm{NaN}$ & $\mathrm{NaN}$ \\
\hline PLCKG29.0+44.6 & 29.01 & 44.56 & 10.25 & A2147 & 0.04 & $\mathrm{NaN}$ & 0.0148 & 0.0021 & $\mathrm{NaN}$ & $\mathrm{NaN}$ & $\mathrm{NaN}$ \\
\hline PLCKG228.5+53.1 & 228.50 & 53.13 & 12.20 & Zw 3179 & 0.14 & $\mathrm{NaN}$ & 0.0022 & 0.0005 & $\mathrm{NaN}$ & $\mathrm{NaN}$ & $\mathrm{NaN}$ \\
\hline PLCKG62.9+43.7 & 62.93 & 43.71 & 10.03 & A2199 & 0.03 & $\mathrm{NaN}$ & 0.0241 & 0.0023 & $\mathrm{NaN}$ & $\mathrm{NaN}$ & $\mathrm{NaN}$ \\
\hline PLCKG206.0-39.5 & 205.96 & -39.48 & 9.26 & MACS J0417.5-1154 & 0.44 & $\mathrm{NaN}$ & 0.0038 & 0.0004 & $\mathrm{NaN}$ & $\mathrm{NaN}$ & $\mathrm{NaN}$ \\
\hline PLCKG336.6-55.4 & 336.59 & -55.45 & 10.29 & A3911 & 0.10 & $\mathrm{NaN}$ & 0.0057 & 0.0006 & $\mathrm{NaN}$ & $\mathrm{NaN}$ & $\mathrm{NaN}$ \\
\hline PLCKG67.2+67.5 & 67.23 & 67.46 & 11.03 & A1914 & 0.17 & $\mathrm{NaN}$ & 0.0057 & 0.0005 & $\mathrm{NaN}$ & $\mathrm{NaN}$ & $\mathrm{NaN}$ \\
\hline PLCKG92.7+73.5 & 92.73 & 73.46 & 11.26 & A1763 & 0.23 & $\mathrm{NaN}$ & 0.0045 & 0.0004 & $\mathrm{NaN}$ & $\mathrm{NaN}$ & $\mathrm{NaN}$ \\
\hline PLCKG146.3-15.6 & 146.33 & -15.59 & 7.10 & CIZA J0254.4+4134 & 0.02 & $\mathrm{NaN}$ & 0.0392 & 0.0060 & $\mathrm{NaN}$ & $\mathrm{NaN}$ & $\mathrm{NaN}$ \\
\hline PLCKG112.5+57.0 & 112.46 & 57.04 & 9.81 & A1767 & 0.07 & $\mathrm{NaN}$ & 0.0053 & 0.0006 & $\mathrm{NaN}$ & $\mathrm{NaN}$ & $\mathrm{NaN}$ \\
\hline PLCKG55.6+31.9 & 55.60 & 31.86 & 9.27 & A2261 & 0.22 & $\mathrm{NaN}$ & 0.0049 & 0.0005 & $\mathrm{NaN}$ & $\mathrm{NaN}$ & $\mathrm{NaN}$ \\
\hline PLCKG58.3+18.6 & 58.28 & 18.59 & 9.19 & CIZA J1825.3+3026 & 0.06 & $\mathrm{NaN}$ & 0.0087 & 0.0009 & $\mathrm{NaN}$ & $\mathrm{NaN}$ & $\mathrm{NaN}$ \\
\hline PLCKG159.9-73.5 & 159.86 & -73.47 & 10.63 & A0209 & 0.21 & $\mathrm{NaN}$ & 0.0053 & 0.0005 & $\mathrm{NaN}$ & $\mathrm{NaN}$ & $\mathrm{NaN}$ \\
\hline PLCKG282.5+65.2 & 282.49 & 65.17 & 8.49 & $\mathrm{ZwCl} 1215.1+0400$ & 0.08 & $\mathrm{NaN}$ & 0.0095 & 0.0012 & $\mathrm{NaN}$ & $\mathrm{NaN}$ & $\mathrm{NaN}$ \\
\hline PLCKG313.4+61.1 & 313.36 & 61.12 & 10.12 & A1689 & 0.18 & $\mathrm{NaN}$ & 0.0071 & 0.0008 & $\mathrm{NaN}$ & $\mathrm{NaN}$ & $\mathrm{NaN}$ \\
\hline PLCKG53.5+59.5 & 53.52 & 59.54 & 8.50 & A2034 & 0.11 & $\mathrm{NaN}$ & 0.0055 & 0.0008 & $\mathrm{NaN}$ & $\mathrm{NaN}$ & $\mathrm{NaN}$ \\
\hline PLCKG244.3-32.1 & 244.34 & hline-32.14 & 8.39 & RBS0653 & 0.28 & $\mathrm{NaN}$ & 0.0029 & 0.0004 & $\mathrm{NaN}$ & $\mathrm{NaN}$ & $\mathrm{NaN}$ \\
\hline PLCKG46.9+56.5 & 46.88 & 56.50 & 9.07 & A2069 & 0.11 & $\mathrm{NaN}$ & 0.0067 & 0.0008 & $\mathrm{NaN}$ & $\mathrm{NaN}$ & $\mathrm{NaN}$ \\
\hline PLCKG294.7-37.0 & 294.67 & -37.03 & 8.64 & RXCJ0303.7-7752 & 0.27 & $\mathrm{NaN}$ & 0.0028 & 0.0004 & $\mathrm{NaN}$ & $\mathrm{NaN}$ & $\mathrm{NaN}$ \\
\hline PLCKG346.6+35.0 & 346.60 & 35.05 & 9.38 & RXCJ1514.9-1523 & 0.22 & $\mathrm{NaN}$ & 0.0048 & 0.0006 & $\mathrm{NaN}$ & $\mathrm{NaN}$ & $\mathrm{NaN}$ \\
\hline PLCKG243.6+67.8 & 243.57 & 67.76 & 8.57 & A1307 & 0.08 & $\mathrm{NaN}$ & 0.0062 & 0.0007 & $\mathrm{NaN}$ & $\mathrm{NaN}$ & $\mathrm{NaN}$ \\
\hline PLCKG166.1+43.4 & 166.13 & 43.39 & 9.23 & A0773 & 0.22 & $\mathrm{NaN}$ & 0.0038 & 0.0004 & $\mathrm{NaN}$ & $\mathrm{NaN}$ & $\mathrm{NaN}$ \\
\hline PLCKG226.2+76.8 & 226.25 & 76.77 & 9.18 & A1413 & 0.14 & $\mathrm{NaN}$ & 0.0058 & 0.0006 & $\mathrm{NaN}$ & $\mathrm{NaN}$ & $\mathrm{NaN}$ \\
\hline PLCKG107.1+65.3 & 107.11 & 65.31 & 8.85 & A1758A & 0.28 & $\mathrm{NaN}$ & 0.0031 & 0.0004 & $\mathrm{NaN}$ & $\mathrm{NaN}$ & $\mathrm{NaN}$ \\
\hline PLCKG42.8+56.6 & 42.83 & 56.62 & 8.36 & A2065 & 0.07 & $\mathrm{NaN}$ & 0.0099 & 0.0011 & $\mathrm{NaN}$ & $\mathrm{NaN}$ & $\mathrm{NaN}$ \\
\hline PLCKG125.6-64.1 & 125.59 & -64.14 & 10.47 & A0119 & 0.04 & $\mathrm{NaN}$ & 0.0141 & 0.0017 & $\mathrm{NaN}$ & $\mathrm{NaN}$ & $\mathrm{NaN}$ \\
\hline PLCKG57.3-45.4 & 57.27 & -45.36 & 8.11 & MACS J2211.7-0349 & 0.40 & $\mathrm{NaN}$ & 0.0032 & 0.0004 & $\mathrm{NaN}$ & $\mathrm{NaN}$ & $\mathrm{NaN}$ \\
\hline PLCKG33.5-48.4 & 33.46 & -48.43 & 9.24 & A2384A & 0.09 & $\mathrm{NaN}$ & 0.0054 & 0.0006 & $\mathrm{NaN}$ & $\mathrm{NaN}$ & $\mathrm{NaN}$ \\
\hline
\end{tabular}


Planck Collaboration: Planck early results. VIII.

Table A.1. continued.

\begin{tabular}{|c|c|c|c|c|c|c|c|c|c|c|c|}
\hline Name & GLON & GLAT & $\overline{S S / N}$ & $\overline{\mathrm{ID}}$ & $\overline{\mathrm{ZZ}}$ & $\overline{\overline{\Theta_{X}}}$ & $\overline{\bar{Y}} \overline{P S X}$ & $\overline{Y_{P S X}^{E R R}}$ & $\overline{\Theta \Theta}$ & $\bar{Y}$ & $\overline{Y^{E T}}$ \\
\hline PLCKG241.8-24.0 & 241.78 & -24.00 & 8.94 & A3378 & 0.14 & $\mathrm{NaN}$ & 0.0038 & 0.0005 & $\mathrm{NaN}$ & $\mathrm{NaN}$ & $\mathrm{NaN}$ \\
\hline PLCKG46.5-49.4 & 46.50 & -49.44 & 8.55 & A2420 & 0.08 & $\mathrm{NaN}$ & 0.0064 & 0.0008 & $\mathrm{NaN}$ & $\mathrm{NaN}$ & $\mathrm{NaN}$ \\
\hline PLCKG304.9+45.5 & 304.90 & 45.45 & 8.99 & A1644 & 0.05 & $\mathrm{NaN}$ & 0.0152 & 0.0018 & $\mathrm{NaN}$ & $\mathrm{NaN}$ & $\mathrm{NaN}$ \\
\hline PLCKG209.6-36.5 & 209.56 & -36.49 & 7.96 & A0496 & 0.03 & $\mathrm{NaN}$ & 0.0162 & 0.0021 & $\mathrm{NaN}$ & $\mathrm{NaN}$ & $\mathrm{NaN}$ \\
\hline PLCKG57.0-55.1 & 56.97 & -55.08 & 8.16 & MACS J2243.3- & 0.45 & $\mathrm{NaN}$ & 0.0029 & 0.0004 & $\mathrm{NaN}$ & $\mathrm{NaN}$ & $\mathrm{NaN}$ \\
\hline PLCKG56.8+36.3 & 56.81 & 36.32 & 9.15 & A2244 & 0.10 & $\mathrm{NaN}$ & 0.0058 & 0.0007 & $\mathrm{NaN}$ & $\mathrm{NaN}$ & $\mathrm{NaN}$ \\
\hline PLCKG57.6+34.9 & 57.61 & 34.94 & 9.54 & A2249 & 0.08 & $\mathrm{NaN}$ & 0.0052 & 0.0007 & $\mathrm{NaN}$ & $\mathrm{NaN}$ & $\mathrm{NaN}$ \\
\hline PLCKG49.2+30.9 & 49.20 & 30.86 & 8.33 & RXC J1720.1+2637 & 0.16 & $\mathrm{NaN}$ & 0.0043 & 0.0005 & $\mathrm{NaN}$ & $\mathrm{NaN}$ & $\mathrm{NaN}$ \\
\hline PLCKG6.7-35.5 & 6.70 & -35.54 & 8.45 & A3695 & 0.09 & $\mathrm{NaN}$ & 0.0059 & 0.0008 & $\mathrm{NaN}$ & $\mathrm{NaN}$ & $\mathrm{NaN}$ \\
\hline PLCKG77.9-26.6 & 77.91 & -26.65 & 8.36 & A2409 & 0.15 & $\mathrm{NaN}$ & 0.0040 & 0.0005 & $\mathrm{NaN}$ & $\mathrm{NaN}$ & $\mathrm{NaN}$ \\
\hline PLCKG8.9-81.2 & 8.94 & -81.24 & 8.39 & A2744 & 0.31 & $\mathrm{NaN}$ & 0.0042 & 0.0005 & $\mathrm{NaN}$ & $\mathrm{NaN}$ & $\mathrm{NaN}$ \\
\hline PLCKG106.7-83.2 & 106.73 & -83.23 & 8.55 & A2813 & 0.29 & $\mathrm{NaN}$ & 0.0036 & 0.0004 & $\mathrm{NaN}$ & $\mathrm{NaN}$ & $\mathrm{NaN}$ \\
\hline PLCKG269.5+26.4 & 269.52 & 26.42 & 8.40 & A1060 & 0.01 & $\mathrm{NaN}$ & 0.0215 & 0.0029 & $\mathrm{NaN}$ & $\mathrm{NaN}$ & $\mathrm{NaN}$ \\
\hline PLCKG180.2+21.0 & 180.24 & 21.05 & 8.36 & MACS J0717.5+3745 & 0.55 & $\mathrm{NaN}$ & .0028 & 0.0004 & $\mathrm{NaN}$ & $\mathrm{NaN}$ & $\mathrm{NaN}$ \\
\hline PLCKG241.7-30.9 & 241.74 & -30.89 & 7.42 & RXCJ0532.9-370 & 0.27 & $\mathrm{NaN}$ & 0.0028 & 0.0004 & $\mathrm{NaN}$ & $\mathrm{NaN}$ & $\mathrm{NaN}$ \\
\hline PLCKG332.9-19.3 & 332.89 & -19.28 & 7.72 & CIZA J1813.3- & 0.15 & $\mathrm{NaN}$ & 0.0043 & 0.0006 & $\mathrm{NaN}$ & $\mathrm{NaN}$ & $\mathrm{JaN}$ \\
\hline+57.2 & 48.05 & 57.18 & 7.14 & A2061 & 0.08 & $\mathrm{NaN}$ & 0.0067 & 0.0010 & $\mathrm{NaN}$ & $\mathrm{NaN}$ & $\mathrm{NaN}$ \\
\hline $2+56.4$ & 139.20 & 56.36 & 7.65 & 51 & 0.32 & $\mathrm{NaN}$ & 0.0012 & 0003 & $\mathrm{NaN}$ & $\mathrm{NaN}$ & $\mathrm{TaN}$ \\
\hline $.7+61.1$ & 306.68 & 61.06 & 8.02 & A1650 & 0.08 & $\mathrm{NaN}$ & .0095 & .0012 & $\mathrm{~N}$ & $\mathrm{JaN}$ & $\mathrm{JaN}$ \\
\hline $.7+17.6$ & 167.66 & 17.65 & 8.11 & $0634.1+$ & 0.17 & $\mathrm{NaN}$ & 0.0045 & .0005 & $\mathrm{NaN}$ & $\mathrm{NaN}$ & $\mathrm{NaN}$ \\
\hline PLCKG49.3+44.4 & 49.34 & 44.38 & 7.40 & A2175 & 0.10 & $\mathrm{NaN}$ & 0.0054 & 0.0009 & $\mathrm{NaN}$ & $\mathrm{NaN}$ & $\mathrm{NaN}$ \\
\hline PLCKG226.2-21.9 & 226.18 & -21.91 & 7.28 & A0550 & 0.10 & $\mathrm{NaN}$ & 0.0047 & 0.0007 & $\mathrm{NaN}$ & $\mathrm{NaN}$ & $\mathrm{NaN}$ \\
\hline PLCKG195.8-24.3 & 195.77 & -24.31 & 7.23 & A0520 & 0.20 & $\mathrm{NaN}$ & 0.0046 & 0.0006 & $\mathrm{NaN}$ & $\mathrm{NaN}$ & $\mathrm{NaN}$ \\
\hline PLCKG253.5-33.7 & 253.48 & -33.72 & 6.73 & A 3343 & 0.19 & $\mathrm{NaN}$ & 0.0022 & 0.0004 & $\mathrm{NaN}$ & $\mathrm{NaN}$ & $\mathrm{NaN}$ \\
\hline PLCKG250.9-36.3 & 250.91 & -36.26 & 8.62 & A 3322 & 0.20 & $\mathrm{NaN}$ & 0.0028 & 0.0004 & $\mathrm{NaN}$ & $\mathrm{NaN}$ & $\mathrm{NaN}$ \\
\hline PLCKG256.5-65.7 & 56.45 & -65.71 & 7.77 & A3016 & 0.22 & $\mathrm{NaN}$ & 0.0029 & 0.0004 & $\mathrm{NaN}$ & $\mathrm{NaN}$ & $\mathrm{NaN}$ \\
\hline PLCKG324.5-45.0 & 324.50 & -44.97 & 6.22 & RBS1847 & 0.10 & $\mathrm{NaN}$ & 0.0039 & 0.0005 & $\mathrm{NaN}$ & $\mathrm{NaN}$ & $\mathrm{NaN}$ \\
\hline $13.8+44.4$ & 113.82 & 44.35 & 7.80 & A1895 & 0.22 & $\mathrm{NaN}$ & 0.0012 & 0.0002 & $\mathrm{NaN}$ & $\mathrm{NaN}$ & $\mathrm{NaN}$ \\
\hline PLCKG125.7+53.9 & 125.71 & 53.86 & 7.36 & 76 & 0.30 & $\mathrm{NaN}$ & 0.0019 & 0.0003 & $\mathrm{NaN}$ & $\mathrm{NaN}$ & $\mathrm{JaN}$ \\
\hline $266.8+25.1$ & 266.84 & 25.08 & 8.19 & 44 & 0.25 & $\mathrm{NaN}$ & 0.0027 & 0.0004 & $\mathrm{NaN}$ & $\mathrm{NaN}$ & $\mathrm{JaN}$ \\
\hline $6+47.0$ & 216.62 & 02 & 7.48 & $8+x-2=$ & 0.38 & & 21 & 04 & $\mathrm{~N}$ & $\mathrm{NaN}$ & $\mathrm{JaN}$ \\
\hline 75.2 & 228.16 & 75.19 & 7.13 & MA & 0.55 & $\mathrm{~N}$ & 16 & 0.0003 & $\mathrm{~N}$ & $\mathrm{NaN}$ & $\mathrm{NaN}$ \\
\hline 4.9 & 42.32 & -34.91 & 7.24 & RXCJ2023.4-5 & 0.23 & $\mathrm{~N}$ & 0.0029 & 0.0004 & $\mathrm{~N}$ & $\mathrm{NaN}$ & $\mathrm{NaN}$ \\
\hline 3-30.5 & 42.82 & -30.46 & 6.01 & A3651 & 0.06 & $\mathrm{NaN}$ & 0.0044 & 0.0009 & $\mathrm{NaN}$ & $\mathrm{NaN}$ & $\mathrm{NaN}$ \\
\hline 1.2-36.5 & 124.22 & -36.49 & 7.74 & A0115 & 0.20 & $\mathrm{NaN}$ & 0.0050 & 0.0007 & $\mathrm{NaN}$ & $\mathrm{NaN}$ & $\mathrm{NaN}$ \\
\hline PLCKG257.3-22.2 & 257.34 & -22.18 & 7.13 & A3399 & 0.20 & $\mathrm{NaN}$ & 0.0019 & 0.0003 & $\mathrm{NaN}$ & $\mathrm{NaN}$ & $\mathrm{NaN}$ \\
\hline PLCKG118.4+39.3 & 118.45 & 39.34 & 6.33 & RXCJ1354.6+ & 0.40 & $\mathrm{NaN}$ & 0.0016 & 0.0003 & $\mathrm{NaN}$ & $\mathrm{NaN}$ & $\mathrm{NaN}$ \\
\hline $18.6+28.6$ & 8.60 & 56 & 6.41 & A2294 & 0.18 & $\mathrm{NaN}$ & 0.0022 & 0.0004 & $\mathrm{NaN}$ & $\mathrm{NaN}$ & $\mathrm{NaN}$ \\
\hline $9.6+78.0$ & 9.64 & .96 & 7.45 & 1443 & 0.27 & $\mathrm{NaN}$ & .0027 & & $\mathrm{~N}$ & $\mathrm{NaN}$ & $\mathrm{NaN}$ \\
\hline $.6+76.7$ & 180.62 & 65 & 7.48 & & 0.21 & & 0.0027 & 0.0004 & & $\mathrm{NaN}$ & $\mathrm{NaN}$ \\
\hline PLC & 2.75 & -56.18 & 6.48 & A3856 & 0.14 & $\mathrm{NaN}$ & 0.0031 & 0.0005 & $\mathrm{NaN}$ & $\mathrm{NaN}$ & $\mathrm{NaN}$ \\
\hline $7.2-27.4$ & 347.19 & -27.35 & 8.19 & ACO S0821 & 0.24 & $\mathrm{NaN}$ & 0.0022 & 0.0004 & $\mathrm{NaN}$ & $\mathrm{NaN}$ & $\mathrm{NaN}$ \\
\hline+29.8 & 61 & 29.80 & 7.47 & & 0.16 & $\mathrm{NaN}$ & & 04 & $\mathrm{~N}$ & $\mathrm{NaN}$ & $\mathrm{NaN}$ \\
\hline & & 92 & 6.98 & & 0.15 & $\mathrm{NaN}$ & & 06 & $\mathrm{NaN}$ & $\mathrm{NaN}$ & $\mathrm{NaN}$ \\
\hline & & -2 & 7.30 & $\mathrm{R}$ & 32 & & & & $\mathrm{iN}$ & $\mathrm{NaN}$ & $\mathrm{JaN}$ \\
\hline .7 & 6 & -26.67 & 7.03 & & 0.15 & N & & & $\mathrm{N}$ & $\mathrm{NaN}$ & $\mathrm{NaN}$ \\
\hline 3.3 & 64 & 63.28 & 7.30 & 37 & 0.13 & $\mathrm{~N}$ & 51 & 0.0 & $\mathrm{NaN}$ & $\mathrm{NaN}$ & $\mathrm{NaN}$ \\
\hline $1+27.2$ & 46.08 & 27.18 & 7.34 & MACS J1731.6+ & 0.39 & $\mathrm{NaN}$ & 0.0 & 0.0 & $\mathrm{NaN}$ & $\mathrm{NaN}$ & $\mathrm{NaN}$ \\
\hline PLCKG49.7-49.5 & .67 & -49.51 & 6.88 & A2426 & 0.10 & $\mathrm{NaN}$ & 0.0038 & 0.0007 & $\mathrm{NaN}$ & $\mathrm{NaN}$ & $\mathrm{NaN}$ \\
\hline PLCKG143.2+65.2 & 143.25 & 65.22 & 7.34 & A1430 & 0.21 & $\mathrm{NaN}$ & 0.0023 & 0.0003 & $\mathrm{NaN}$ & $\mathrm{NaN}$ & $\mathrm{NaN}$ \\
\hline PLCKG296.4-32.5 & 6.41 & -32.49 & 7.20 & ACO S0405 & 0.06 & $\mathrm{NaN}$ & 0.0044 & 0.0007 & $\mathrm{NaN}$ & $\mathrm{NaN}$ & $\mathrm{NaN}$ \\
\hline $69.3-49.9$ & 269.31 & -49.88 & 6.51 & A3126 & 0.09 & $\mathrm{NaN}$ & 0.0040 & 0.0007 & $\mathrm{NaN}$ & $\mathrm{NaN}$ & $\mathrm{NaN}$ \\
\hline PLC & .29 & -31.03 & 6.19 & RXC J2228.6+20 & 0.41 & $\mathrm{NaN}$ & 0.0021 & 0.0004 & $\mathrm{NaN}$ & $\mathrm{NaN}$ & $\mathrm{NaN}$ \\
\hline PLCKG304.7-31.7 & 304.67 & -31.67 & 6.37 & A4023 & 0.19 & $\mathrm{NaN}$ & 0.0020 & 0.0004 & $\mathrm{NaN}$ & $\mathrm{NaN}$ & $\mathrm{NaN}$ \\
\hline PLCKG39.9-40.0 & 39.86 & -39.99 & 6.32 & A2345 & 0.18 & $\mathrm{NaN}$ & 0.0031 & 0.0005 & $\mathrm{NaN}$ & $\mathrm{NaN}$ & $\mathrm{NaN}$ \\
\hline PLCKG56.0-34.9 & 5.98 & -34.89 & 7.03 & A2355 & 0.12 & $\mathrm{NaN}$ & 0.0036 & 0.0005 & $\mathrm{NaN}$ & $\mathrm{NaN}$ & $\mathrm{NaN}$ \\
\hline PLCKG303.8+33.7 & 303.76 & 66 & 6.05 & A3528S & 0.05 & $\mathrm{NaN}$ & 0.0085 & 0.0014 & $\mathrm{NaN}$ & $\mathrm{NaN}$ & $\mathrm{NaN}$ \\
\hline $.7+53.5$ & 163.72 & 3 & 7.46 & A0980 & 0.16 & $\mathrm{NaN}$ & 0.0030 & 0.0004 & $\mathrm{NaN}$ & $\mathrm{NaN}$ & $\mathrm{NaN}$ \\
\hline PLCKG318.1-29.6 & & -29.58 & 6.63 & CJ1947.3-7 & 0.22 & $\mathrm{NaN}$ & & & $\mathrm{NaN}$ & $\mathrm{NaN}$ & $\mathrm{NaN}$ \\
\hline PLCKG244.7+32.5 & & & 6.27 & & 0.15 & $\mathrm{NaN}$ & & & $\mathrm{NaN}$ & $\mathrm{NaN}$ & $\mathrm{NaN}$ \\
\hline $.5+52.4$ & 284.46 & 52.44 & 7.27 & J1206.2-C & 0.44 & $\mathrm{NaN}$ & 0.0029 & 0.0004 & $\mathrm{NaN}$ & $\mathrm{NaN}$ & $\mathrm{NaN}$ \\
\hline PLCKG260.0-63.4 & 260.03 & -63.44 & 7.29 & RXCJ0232.2-4420 & 0.28 & $\mathrm{NaN}$ & 0.0024 & 0.0004 & $\mathrm{NaN}$ & $\mathrm{NaN}$ & $\mathrm{NaN}$ \\
\hline & 2.97 & -56.05 & 6.79 & A3112 & 0.08 & $\mathrm{NaN}$ & 0.0047 & 0.0007 & $\mathrm{NaN}$ & $\mathrm{NaN}$ & $\mathrm{NaN}$ \\
\hline & & & 6.39 & & 0.02 & $\mathrm{NaN}$ & 0.0146 & 0.0029 & $\mathrm{NaN}$ & $\mathrm{NaN}$ & $\mathrm{NaN}$ \\
\hline $26+302$ & 278.61 & & & 300 & 0.31 & & 0.0035 & 0.0005 & $\mathrm{NaN}$ & $\mathrm{NaN}$ & $\mathrm{NaN}$ \\
\hline $5-261$ & 2465 & -26.06 & 6.5 & 3376 & 0.05 & $\mathrm{NaN}$ & 0.0053 & 0.0010 & $\mathrm{NaN}$ & $\mathrm{NaN}$ & $\mathrm{NaN}$ \\
\hline PLCKG114.3+64.9 & 114.34 & 64.87 & 6.18 & A1703 & 0.28 & $\mathrm{NaN}$ & 0.0020 & 0.0003 & $\mathrm{NaN}$ & $\mathrm{NaN}$ & $\mathrm{NaN}$ \\
\hline
\end{tabular}


Table A.1. continued.

\begin{tabular}{|c|c|c|c|c|c|c|c|c|c|c|c|}
\hline Name & GLON & GLAT & $S / N$ & ID & $\mathrm{z}$ & $\Theta_{X}$ & $Y_{P S X}$ & $Y_{P S X}^{E R R}$ & $\Theta$ & $Y$ & $Y^{E R R}$ \\
\hline PLCKG80.4-33.2 & 80.38 & -33.20 & 6.06 & A2443 & 0.11 & $\mathrm{NaN}$ & 0.0039 & $\frac{P S x}{0.0006}$ & $\mathrm{NaN}$ & $\mathrm{NaN}$ & $\mathrm{NaN}$ \\
\hline PLCKG249.9-39.9 & 249.88 & -39.87 & 6.25 & A3292 & 0.15 & $\mathrm{NaN}$ & 0.0018 & 0.0004 & $\mathrm{NaN}$ & $\mathrm{NaN}$ & $\mathrm{NaN}$ \\
\hline PLCKG182.6+55.8 & 182.64 & 55.82 & 6.81 & A0963 & 0.21 & $\mathrm{NaN}$ & 0.0019 & 0.0004 & $\mathrm{NaN}$ & $\mathrm{NaN}$ & $\mathrm{NaN}$ \\
\hline PLCKG62.4-46.4 & 62.42 & -46.41 & 6.33 & A2440 & 0.09 & $\mathrm{NaN}$ & 0.0041 & 0.0007 & $\mathrm{NaN}$ & $\mathrm{NaN}$ & $\mathrm{NaN}$ \\
\hline PLCKG8.4-56.4 & 8.45 & -56.36 & 6.39 & A3854 & 0.15 & $\mathrm{NaN}$ & 0.0024 & 0.0005 & $\mathrm{NaN}$ & $\mathrm{NaN}$ & $\mathrm{NaN}$ \\
\hline PLCKG229.2-17.2 & 229.22 & -17.25 & 6.18 & RXCJ0616.3-2156 & 0.17 & $\mathrm{NaN}$ & 0.0031 & 0.0005 & $\mathrm{NaN}$ & $\mathrm{NaN}$ & $\mathrm{NaN}$ \\
\hline PLCKG341.0+35.1 & 340.96 & 35.12 & 6.61 & ACO S0780 & 0.24 & $\mathrm{NaN}$ & 0.0030 & 0.0007 & $\mathrm{NaN}$ & $\mathrm{NaN}$ & $\mathrm{NaN}$ \\
\hline PLCKG218.9+35.5 & 218.86 & 35.51 & 6.87 & A0750 & 0.18 & $\mathrm{NaN}$ & 0.0027 & 0.0005 & $\mathrm{NaN}$ & $\mathrm{NaN}$ & $\mathrm{NaN}$ \\
\hline PLCKG165.1+54.1 & 165.09 & 54.12 & 6.34 & A0990 & 0.14 & $\mathrm{NaN}$ & 0.0027 & 0.0005 & $\mathrm{NaN}$ & $\mathrm{NaN}$ & $\mathrm{NaN}$ \\
\hline PLCKG161.4+26.2 & 161.44 & 26.23 & 6.63 & A0576 & 0.04 & $\mathrm{NaN}$ & 0.0076 & 0.0012 & $\mathrm{NaN}$ & $\mathrm{NaN}$ & $\mathrm{NaN}$ \\
\hline PLCKG295.3+23.3 & 295.33 & 23.34 & 6.11 & RXCJ1215.4-3900 & 0.12 & $\mathrm{NaN}$ & 0.0042 & 0.0008 & $\mathrm{NaN}$ & $\mathrm{NaN}$ & $\mathrm{NaN}$ \\
\hline PLCKG280.2+47.8 & 280.20 & 47.82 & 7.06 & A1391 & 0.16 & $\mathrm{NaN}$ & 0.0042 & 0.0006 & $\mathrm{NaN}$ & $\mathrm{NaN}$ & $\mathrm{NaN}$ \\
\hline PLCKG0.4-41.8 & 0.44 & -41.84 & 6.55 & A3739 & 0.17 & $\mathrm{NaN}$ & 0.0025 & 0.0005 & $\mathrm{NaN}$ & $\mathrm{NaN}$ & $\mathrm{NaN}$ \\
\hline PLCKG195.6+44.1 & 195.62 & 44.05 & 6.88 & A0781 & 0.30 & $\mathrm{NaN}$ & 0.0017 & 0.0003 & $\mathrm{NaN}$ & $\mathrm{NaN}$ & $\mathrm{NaN}$ \\
\hline PLCKG241.9+51.5 & 241.86 & 51.53 & 6.96 & A1066 & 0.07 & $\mathrm{NaN}$ & 0.0024 & 0.0007 & $\mathrm{NaN}$ & $\mathrm{NaN}$ & $\mathrm{NaN}$ \\
\hline PLCKG81.0-50.9 & 81.00 & -50.91 & 6.76 & A2552 & 0.30 & $\mathrm{NaN}$ & 0.0026 & 0.0005 & $\mathrm{NaN}$ & $\mathrm{NaN}$ & $\mathrm{NaN}$ \\
\hline PLCKG304.5+32.4 & 304.50 & 32.44 & 6.86 & A3532 & 0.06 & $\mathrm{NaN}$ & 0.0068 & 0.0015 & $\mathrm{NaN}$ & $\mathrm{NaN}$ & $\mathrm{NaN}$ \\
\hline PLCKG306.8+58.6 & 306.80 & 58.61 & 6.81 & A1651 & 0.08 & $\mathrm{NaN}$ & 0.0077 & 0.0012 & $\mathrm{NaN}$ & $\mathrm{NaN}$ & $\mathrm{NaN}$ \\
\hline PLCKG172.9+65.3 & 172.89 & 65.32 & 6.30 & A1190 & 0.08 & $\mathrm{NaN}$ & 0.0030 & 0.0006 & $\mathrm{NaN}$ & $\mathrm{NaN}$ & $\mathrm{NaN}$ \\
\hline PLCKG99.0+24.9 & 98.95 & 24.86 & 6.49 & A2312 & 0.09 & $\mathrm{NaN}$ & 0.0022 & 0.0004 & $\mathrm{NaN}$ & $\mathrm{NaN}$ & $\mathrm{NaN}$ \\
\hline PLCKG247.2-23.3 & 247.17 & -23.33 & 6.19 & ACO S0579 & 0.15 & $\mathrm{NaN}$ & 0.0019 & 0.0004 & $\mathrm{NaN}$ & $\mathrm{NaN}$ & $\mathrm{NaN}$ \\
\hline PLCKG176.3-35.1 & 176.28 & -35.05 & 6.38 & $2 \mathrm{~A} 0335+096$ & 0.03 & $\mathrm{NaN}$ & 0.0117 & 0.0025 & $\mathrm{NaN}$ & $\mathrm{NaN}$ & $\mathrm{NaN}$ \\
\hline PLCKG57.9+27.6 & 57.93 & 27.64 & 6.13 & $\mathrm{ZwCl} 1742.1+3306$ & 0.08 & $\mathrm{NaN}$ & 0.0037 & 0.0008 & $\mathrm{NaN}$ & $\mathrm{NaN}$ & $\mathrm{NaN}$ \\
\hline PLCKG275.2+43.9 & 275.22 & 43.92 & 6.29 & A1285 & 0.11 & $\mathrm{NaN}$ & 0.0044 & 0.0008 & $\mathrm{NaN}$ & $\mathrm{NaN}$ & $\mathrm{NaN}$ \\
\hline PLCKG96.9+52.5 & 96.85 & 52.47 & 6.12 & A1995 & 0.32 & $\mathrm{NaN}$ & 0.0015 & 0.0003 & $\mathrm{NaN}$ & $\mathrm{NaN}$ & $\mathrm{NaN}$ \\
\hline PLCKG72.8-18.7 & 72.80 & -18.72 & 10.10 & $\mathrm{ZwCl} 2120.1+2256$ & 0.14 & $\mathrm{NaN}$ & $\mathrm{NaN}$ & $\mathrm{NaN}$ & 37.22 & 0.0052 & 0.0010 \\
\hline PLCKG239.3-26.0 & 239.29 & -26.00 & 8.64 & MACS J0553.4-3342 & 0.41 & $\mathrm{NaN}$ & $\mathrm{NaN}$ & $\mathrm{NaN}$ & 17.22 & 0.0026 & 0.0006 \\
\hline PLCKG8.3-64.8 & 8.30 & -64.76 & 8.47 & AC114Northern & 0.31 & $\mathrm{NaN}$ & $\mathrm{NaN}$ & $\mathrm{NaN}$ & 43.83 & 0.0048 & 0.0010 \\
\hline PLCKG94.0+27.4 & 94.02 & 27.43 & 6.92 & $\mathrm{H} 1821+643$ & 0.30 & $\mathrm{NaN}$ & $\mathrm{NaN}$ & $\mathrm{NaN}$ & 40.25 & 0.0030 & 0.0014 \\
\hline PLCKG157.4+30.3 & 157.43 & 30.34 & 6.18 & RXJ0748.7+5941 & $\mathrm{NaN}$ & $\mathrm{NaN}$ & $\mathrm{NaN}$ & $\mathrm{NaN}$ & 22.43 & 0.0025 & 0.0014 \\
\hline PLCKG345.4-39.3 & 345.41 & -39.34 & 7.10 & ABELL3716S & 0.04 & $\mathrm{NaN}$ & $\mathrm{NaN}$ & $\mathrm{NaN}$ & 118.59 & 0.0109 & 0.0032 \\
\hline PLCKG53.4-36.3 & 53.44 & -36.27 & 6.88 & MACS J2135.2-0102 & 0.32 & $\mathrm{NaN}$ & $\mathrm{NaN}$ & $\mathrm{NaN}$ & 8.07 & 0.0018 & 0.0003 \\
\hline PLCKG271.5-56.6 & 271.50 & -56.56 & 6.71 & ACO S0295 & 0.30 & $\mathrm{NaN}$ & $\mathrm{NaN}$ & $\mathrm{NaN}$ & 20.26 & 0.0025 & 0.0007 \\
\hline PLCKG86.0+26.7 & 86.00 & 26.71 & 6.55 & A2302 & 0.18 & $\mathrm{NaN}$ & $\mathrm{NaN}$ & $\mathrm{NaN}$ & 56.62 & 0.0043 & 0.0019 \\
\hline PLCKG96.9+24.2 & 96.88 & 24.22 & 6.24 & ZwCl1856.8+6616 & $\mathrm{NaN}$ & $\mathrm{NaN}$ & $\mathrm{NaN}$ & $\mathrm{NaN}$ & 20.64 & 0.0015 & 0.0005 \\
\hline PLCKG164.6+46.4 & 164.61 & 46.39 & 6.06 & $\mathrm{ZwCl0934.8+5216}$ & $\mathrm{NaN}$ & $\mathrm{NaN}$ & $\mathrm{NaN}$ & $\mathrm{NaN}$ & 16.50 & 0.0018 & 0.0006 \\
\hline PLCKG285.0-23.7 & 284.99 & -23.71 & 11.48 & null & 0.44 & $\mathrm{NaN}$ & 0.0023 & 0.0002 & $\mathrm{NaN}$ & $\mathrm{NaN}$ & $\mathrm{NaN}$ \\
\hline PLCKG287.0+32.9 & 286.99 & 32.92 & 10.62 & null & 0.39 & $\mathrm{NaN}$ & 0.0061 & 0.0006 & $\mathrm{NaN}$ & $\mathrm{NaN}$ & $\mathrm{NaN}$ \\
\hline PLCKG171.9-40.7 & 171.95 & -40.66 & 10.61 & null & 0.39 & $\mathrm{NaN}$ & 0.0062 & 0.0006 & $\mathrm{NaN}$ & $\mathrm{NaN}$ & $\mathrm{NaN}$ \\
\hline PLCKG271.2-31.0 & 271.20 & -30.97 & 8.48 & null & 0.27 & $\mathrm{NaN}$ & 0.0020 & 0.0002 & $\mathrm{NaN}$ & $\mathrm{NaN}$ & $\mathrm{NaN}$ \\
\hline PLCKG262.7-40.9 & 262.71 & -40.91 & 8.27 & ACT-CLJ0438-5419 & 0.37 & $\mathrm{NaN}$ & 0.0021 & 0.0002 & $\mathrm{NaN}$ & $\mathrm{NaN}$ & $\mathrm{NaN}$ \\
\hline PLCKG308.3-20.2 & 308.32 & -20.23 & 8.26 & null & 0.39 & $\mathrm{NaN}$ & $\mathrm{NaN}$ & $\mathrm{NaN}$ & 32.81 & 0.0049 & 0.0013 \\
\hline PLCKG277.8-51.7 & 277.75 & -51.73 & 7.40 & null & 0.21 & $\mathrm{NaN}$ & 0.0027 & 0.0003 & $\mathrm{NaN}$ & $\mathrm{NaN}$ & $\mathrm{NaN}$ \\
\hline PLCKG286.6-31.3 & 286.59 & -31.25 & 6.89 & null & 0.30 & $\mathrm{NaN}$ & 0.0026 & 0.0004 & $\mathrm{NaN}$ & $\mathrm{NaN}$ & $\mathrm{NaN}$ \\
\hline PLCKG292.5+22.0 & 292.52 & 21.99 & 6.88 & null & 0.35 & $\mathrm{NaN}$ & 0.0037 & 0.0006 & $\mathrm{NaN}$ & $\mathrm{NaN}$ & $\mathrm{NaN}$ \\
\hline PLCKG337.1-26.0 & 337.09 & -25.97 & 6.59 & null & 0.48 & $\mathrm{NaN}$ & $\mathrm{NaN}$ & $\mathrm{NaN}$ & 31.56 & 0.0034 & 0.0008 \\
\hline PLCKG285.6-17.2 & 285.64 & -17.25 & 6.35 & null & 0.12 & $\mathrm{NaN}$ & 0.0016 & 0.0003 & $\mathrm{NaN}$ & $\mathrm{NaN}$ & $\mathrm{NaN}$ \\
\hline PLCKG225.9-20.0 & 225.93 & -20.00 & 8.07 & null & $\mathrm{NaN}$ & $\mathrm{NaN}$ & $\mathrm{NaN}$ & $\mathrm{NaN}$ & 28.21 & 0.0040 & 0.0011 \\
\hline PLCKG255.6-46.2 & 255.63 & -46.17 & 8.46 & null & $\mathrm{NaN}$ & $\mathrm{NaN}$ & $\mathrm{NaN}$ & $\mathrm{NaN}$ & 31.23 & 0.0026 & 0.0006 \\
\hline PLCKG304.8-41.4 & 304.84 & -41.42 & 7.58 & null & $\mathrm{NaN}$ & $\mathrm{NaN}$ & $\mathrm{NaN}$ & $\mathrm{NaN}$ & 21.68 & 0.0022 & 0.0006 \\
\hline PLCKG121.1+57.0 & 121.12 & 57.01 & 6.66 & null & $\mathrm{NaN}$ & $\mathrm{NaN}$ & $\mathrm{NaN}$ & $\mathrm{NaN}$ & 17.99 & 0.0016 & 0.0004 \\
\hline PLCKG283.2-22.9 & 283.16 & -22.93 & 6.03 & null & $\mathrm{NaN}$ & $\mathrm{NaN}$ & $\mathrm{NaN}$ & $\mathrm{NaN}$ & 26.73 & 0.0018 & 0.0008 \\
\hline PLCKG139.6+24.2 & 139.60 & 24.19 & 7.21 & null & $\mathrm{NaN}$ & $\mathrm{NaN}$ & $\mathrm{NaN}$ & $\mathrm{NaN}$ & 24.52 & 0.0032 & 0.0013 \\
\hline PLCKG189.8-37.2 & 189.85 & -37.24 & 6.71 & null & $\mathrm{NaN}$ & $\mathrm{NaN}$ & $\mathrm{NaN}$ & $\mathrm{NaN}$ & 62.50 & 0.0080 & 0.0021 \\
\hline PLCKG264.4+19.5 & 264.42 & 19.48 & 6.15 & null & $\mathrm{NaN}$ & $\mathrm{NaN}$ & $\mathrm{NaN}$ & $\mathrm{NaN}$ & 32.25 & 0.0028 & 0.0010 \\
\hline PLCKG115.7+17.5 & 115.72 & 17.53 & 6.78 & null & $\mathrm{NaN}$ & $\mathrm{NaN}$ & $\mathrm{NaN}$ & $\mathrm{NaN}$ & 17.48 & 0.0025 & 0.0008 \\
\hline
\end{tabular}

